\author{
Universidade de São Paulo \\ Instituto de Astronomia, Geofísica e Ciências Atmosféricas \\ Departamento de Ciências Atmosféricas
}

André Seiji Wakate Teruya

\title{
Energética dos Modos Normais da Dinâmica Atmosférica Não-Hidrostática
}

São Paulo 

André Seiji Wakate Teruya

\section{Energética dos Modos Normais da Dinâmica Atmosférica Não-Hidrostática}

Dissertação apresentada ao Departamento de Ciências Atmosféricas do Instituto de Astronomia, Geofísica e Ciências Atmosféricas da Universidade de São Paulo como requisito parcial para a obtenção do título de Mestre em Ciências.

Área de Concentração: Meteorologia Orientador: Prof. Dr. Carlos Frederico Mendonça Raupp

São Paulo 

Aos meus avós, Teruya Kentaro (in memoriam), Teruya Emiko, Wakate Tadao e Wakate Fumiko. 


\section{Agradecimentos}

Aos meu pais Roberto e Lenir e ao meu irmão Key, pelo amor, carinho e apoio ao longo da minha vida;

À minha namorada Kaori, pelo suporte e amor concedidos nestes últimos quatro anos;

Ao Prof. Carlos Frederico Mendonça Raupp, pela orientação e incentivo durante a execução deste projeto;

Ao Prof. Adilson Wagner Gandu, que me apresentou as diversas áreas de pesquisa do IAG.

Aos Professores do IAG, que de alguma forma contribuíram para minha formação;

Aos amigos e colegas do Instituto de Física (IF), Instituto de Matemática e Estatística (IME) e do Instituto de Astronomia, Geofísica e Ciências Atmosféricas (IAG);

Ao Conselho Nacional de Desenvolvimento Científico e Tecnológico (CNPQ), pelo apoio financeiro durante os dois primeiros meses deste projeto;

À Fundação de Amparo à Pesquisa do Estado de São Paulo (FAPESP), pelo apoio financeiro durante o período restante do desenvolvimento deste trabalho, sob o projeto $\mathrm{n}^{o}$ : 2012/06388-8.

Esta tese/dissertação foi escrita em IATEX com a classe IAGTESE, para teses e dissertações do IAG. 


\section{Resumo}

O presente trabalho analisou a energética linear e fracamente não-linear dos modos normais de um modelo atmosférico global, baroclínico, compressível, não-hidrostático e incluindo a geometria esférica da Terra. Os modos normais correspondem às soluções de ondas lineares livres das equações governantes do modelo em questão na presença de um estado básico em repouso e em equilíbrio hidrostático. No entanto, para simplificar a análise matemática, considerou-se ainda um estado básico isotérmico.

Esses modos foram determinados seguindo o procedimento descrito no artigo de Kasahara e Qian (2000). Em seguida, foi computada a energia total bem como cada uma de suas componentes para os modos acústico-inerciais e de gravidade-inerciais e analisada a variação dessas energias com o número de onda zonal.

Da energética dos modos, foi possível concluir que os efeitos não-hidrostáticos são mais acentuados nos modos gravito-inerciais mais curtos. Além disso, foi mostrado que a energia dos modos acústico-inerciais está concentrada nas energias do tipo elástica e cinética vertical. Entretanto, a energia cinética vertical diminui, enquanto a energia elástica aumenta com o aumento do número de onda zonal.

Para aprofundar o estudo sobre a dinâmica dos modos normais do modelo em questão, realizou-se o estudo das interações fracamente não-lineares permitidas pelo modelo. Mais especificamente, foram analisadas as trocas de energia de um tripleto ressonante composto por dois modos acústico-inerciais e um modo gravito-inercial. Em experimentos numéricos foi possível mostrar que mesmo os modos de altíssima frequência (com períodos de oscilação temporal de aproximadamente 15 segundos) podem realizar trocas significativas de energia cujo período de oscilação característico pode variar de algumas horas até um período de aproximadamente vinte dias. Além disso, mostrou-se que essas trocas de energia modulam 
as perturbações dos campos de vento e pressão. 


\section{Abstract}

In the present monograph the linear and weakly nonlinear energetics of the normal modes of a nonhydrostatic, compressible, and baroclinic global atmospheric model was analized. The normal modes are the linear free wave solutions of a dynamical system linearized around a basic state that is hydrostatic and at rest. Nevertheless, it was assumed an isothermal basic state, so the mathematical treatment of the problem becomes less cumbersome.

The eigenfunctions of this set of linearized equations are obtained by the method proposed by Kasahara e Qian (2000). Then the total energy associated with the perturbations and its components are derived from the governing equations in their Eulerian representation. Next, the functional relation between these energies and the zonal wavenumber of the inertia-gravity and acoustic-inertia modes is analyzed.

From the normal mode's energetics it is shown that the nonhydrostatic effects are more apparent on the shorter inertia-gravity modes. It is also shown that the vertical kinetic energy and the elastic energy of the acoustic-inertia modes are the dominant kind of energy of these modes. Although as the zonal wavenumber becomes bigger, the importance of the elastic energy grows and the vertical kinetic energy becomes less significant.

The weakly nonlinear interactions between the normal modes of the model considered in this monograph are also analyzed. For instance, the nonlinear energy exchanges of the resonant triads, composed of two acoustic-inertia modes and one inertia-gravity mode. From numerical experiments it is shown that high-frequency modes (i.e., modes with period of oscillation around 15 seconds) could significantly exchange energy, whereas the period of these exchanges could range from a couple hours to almost twenty days long. It's also shown that those energy exchanges modulate the perturbations on the wind and pressure's 
field. 


\section{Lista de Figuras}

1.1 Diagrama que mostra a relação entre quatro modelos atmosféricos globais e como os mesmos estão relacionados com o sistema de equações completo. .

3.1 Diagrama esquematizando o método iterativo descrito no texto. A linha tracejada indica a altura equivalente inicial. A curva representando a frequência de Hough é a obtida em (3.60). A frequência não-hidrostática é obtida em

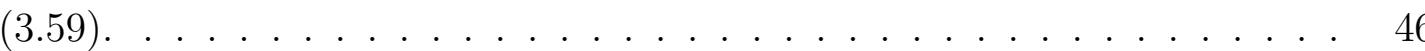

3.2 Relação de dispersão para as oscilações de primeira e terceira espécies.) . 53

3.3 Diagrama da altura equivalente para as oscilações de primeira e terceira

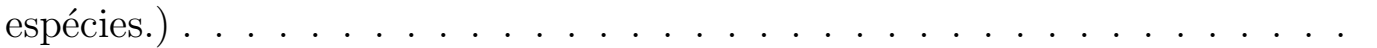

3.4 Energética dos modos acústico-inerciais com índice meridional $l=0$ e modo vertical $k=1.) \ldots \ldots \ldots \ldots \ldots \ldots$

3.5 Energética dos modos gravito-inerciais com índice meridional $l=0$ e modo vertical $k=1.) \ldots \ldots \ldots \ldots \ldots \ldots$

3.6 Energética, normalizada em relação à energia total, dos modos acústicoinerciais com índice meridional $l=0$ e modo vertical $k=1$.) . . . . . . .

3.7 Energética, normalizada em relação à energia total, dos modos gravitoinerciais com índice meridional $l=0$ e modo vertical $k=1$.) . . . . . . .

3.8 Energética, normalizada em relação à energia total, dos modos acústicoinerciais com índice meridional $l=0$ e modo vertical $k=1.) \ldots \ldots$

3.9 Energética, normalizada em relação à energia total, dos modos gravitoinerciais com índice meridional $l=0$ e modo vertical $k=1$.) . . . . . . .

3.10 Energética, normalizada em relação à energia total, dos modos gravitoinerciais com índice meridional $l=2$ e modo vertical $k=1$.) . . . . . . . 
4.1 As figuras acima são apenas diagramas representando método utilizado para obter os tripletos ressonantes. Os valores nos eixos são apenas ilustrativos. Na figura 4.1a temos a relação de dispersão dos modos gravito-inerciais (curva verde) e dos modos acústico-inerciais (curva vermelha) . A curva $N$ representa a frequência de Brunt-Väisälä (curva azul), que separa dos dois regimes citados. Na figura $4.1 \mathrm{~b}$ temos a mesma figura, mas agora a origem da relação de dispersão dos modos acústico inerciais está deslocada para

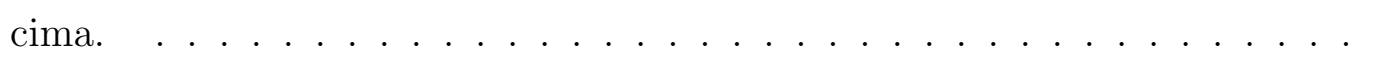

4.2 Nesta figura temos as trocas de energias de um tripleto ressonante referentes ao primeiro experimento $(0 \ll \tilde{m}<1)$. Note que o modo a da figura representa o modo $a$ do texto. . . . . . . . . . . . . . .

4.3 Nesta figura temos as trocas de energias de um tripleto ressonante referentes ao segundo experimento $(0<\tilde{m} \ll 1)$. Note que o modo a da figura representa o modo a do texto. . . . . . . . . . . . . .

4.4 Nesta figura temos as trocas de energias de um tripleto ressonante referentes ao terceiro experimento $(0<\tilde{m}<1)$. Note que o modo $a$ da figura representa o modo a do texto. . . . . . . . . . . . . .

4.5 Evolução temporal da perturbação do campo do vento vertical em $\phi=10^{\circ} \mathrm{S}$, no nível vertical $z=9000 \mathrm{~m}$ e da perturbação do campo do vento zonal em $\phi=0^{\circ}$, no nível vertical $z=4500 \mathrm{~m}$. A velocidade está em $\mathrm{m} / \mathrm{s}$ e o tempo está em dias. Não é possível ver a oscilação interna ao envoltório devido a escala de tempo do gráfico. . . . . . . . . . . . . . . . . . 


\section{Lista de Tabelas}

4.1 Valores do número de onda zonal, índice meridional, modo vertical e o tipo de onda de cada um dos elementos do tripleto analisado, assim como suas respectivas frequências temporais. Os modos são caracterizados, da esquerda para direita, pelo número de onda zonal, índice meridional, modo vertical e o tipo de onda, i.e., acústico-inercial (AI) ou gravito-inercial(GI). A frequência $\sigma$ dos modos está em $\mathrm{cHz}\left(10^{-2} \mathrm{~Hz}\right)$. Os valores das frequências apresentam três algarismos significativos para ressaltar o fato de que o tripleto satisfaz aproximadamente a condição de ressonância

4.2 Valor numérico dos coeficientes de interação dos modos que compõem o tripleto ressonante da Tabela 4.1 . . . . . . . . . . . . . . . . . . . . 76

4.3 Valor numérico das normas (em Joules) dos modos que compõem o tripleto ressonante da Tabela 4.1 . . . . . . . . . . . . . . . . . . . . . . .

4.4 Valores das amplitudes iniciais utilizadas no experimento numérico ilustrado na Figura 4.2. As variáveis $u_{s}, w_{s}$ e $p_{s}$ representam os maiores valores, em superfície, das pertubações da velocidade horizontal, vertical e pressão, respectivamente. Esses valores estão associados à amplitude inicial de cada um dos modos. As velocidades estão em m/s e a pressão está em hPa . . . 78

4.5 Esta tabela refere-se ao experimento ilustrado na Figura 4.2 e é apenas a continuação da Tabela 4.4. As variáveis $u_{T}, w_{T}$ e $p_{T}$ são os maiores valores, no topo da atmosfera, das perturbações da velocidade horizontal, vertical e pressão, respectivamente. As velocidades estão em m/s e a pressão está em hPa. E $\tilde{m}$ é o parâmetro dado pela expressão 4.20 . . . . . . . . . . . . . 79 
4.6 Valores das amplitudes iniciais utilizadas no experimento numérico ilustrado na Figura 4.3. As variáveis $u_{s}, w_{s}$ e $p_{s}$ representam os maiores valores, em superfície, das pertubações da velocidade horizontal, vertical e pressão, respectivamente. Esses valores estão associados à amplitude inicial de cada um dos modos. As velocidades estão em m/s e a pressão está em hPa . . .

4.7 Esta tabela refere-se ao experimento ilustrado na Figura 4.3 e é apenas a continuação da Tabela 4.6. As variáveis $u_{T}, w_{T}$ e $p_{T}$ são os maiores valores, no topo da atmosfera, das perturbações da velocidade horizontal, vertical e pressão, respectivamente. As velocidades estão em m/s e a pressão está em hPa. E $\tilde{m}$ é o parâmetro dado pela expressão 4.20 . . . . . . . . . . . . .

4.8 Valores das amplitudes iniciais utilizadas no experimento numérico ilustrado na Figura 4.4. As variáveis $u_{s}, w_{s}$ e $p_{s}$ representam os maiores valores, em superfície, das pertubações da velocidade horizontal, vertical e pressão, respectivamente. Esses valores estão associados à amplitude inicial de cada um dos modos. As velocidades estão em m/s e a pressão está em hPa . . .

4.9 Esta tabela refere-se ao experimento ilustrado na Figura 4.4 e é apenas a continuação da Tabela 4.8. As variáveis $u_{T}, w_{T}$ e $p_{T}$ são os maiores valores, no topo da atmosfera, das perturbações da velocidade horizontal, vertical e pressão, respectivamente. As velocidades estão em $\mathrm{m} / \mathrm{s}$ e a pressão está em hPa. E $\tilde{m}$ é o parâmetro dado pela expressão 4.20 . . . . . . . . . . . . . 80 


\section{Sumário}

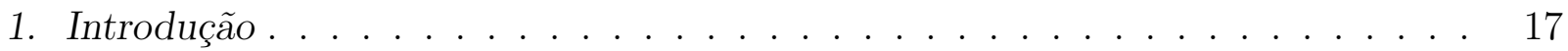

1.1 Equações Aproximadas da Dinâmica dos Fluidos Geofísicos . . . . . . . . . 19

1.2 Modelos Não-Hidrostáticos e Quase-Hidrostáticos . . . . . . . . . . . . . 20

1.2.1 Modos Normais . . . . . . . . . . . . . . . . . 22

1.3 Objetivos e Organização do Texto . . . . . . . . . . . . . . . . . . . . . 24

2. Equações Governantes e Teoria da Perturbação . . . . . . . . . . . . . . . . . 25

3. Modos Normais do Sistema Linearizado . . . . . . . . . . . . . . . . . . . . . . 31

3.1 Modos Normais do Sistema Linearizado . . . . . . . . . . . . . . . . . . . 31

3.1.1 Problema de autovalor ................... 35

3.1.2 Solução da estrutura horizontal(Funções de Hough) . . . . . . . . . 36

3.1.3 Determinação das autofrequências e alturas equivalentes, no caso hidrostático ....................... 43

3.1.4 Determinação das autofrequências e alturas equivalentes, no caso não-hidrostático . . . . . . . . . . . . . . . . . . 43

3.1.5 Ortogonalidade e energia dos modos . . . . . . . . 48

3.1.6 Cálculo das Energias . . . . . . . . . . . . . . . . . . . 50

3.2 Energética dos Modos Acústico-Inerciais e Gravito-Inerciais . . . . . . . . . 51

3.2.1 Relação de Dispersão e Diagramas da Altura Equivalente . . . . . . 52

3.2.2 Energética dos Modos Não-Hidrostáticos . . . . . . . . . 52

3.2.3 Comparação com a Energética dos Modos Hidrostáticos . . . . . . . 58

3.2.4 Resumo sobre a Energética Linear . . . . . . . . . . . . . . 59 
4. Energética Não-Linear do Modos Normais . . . . . . . . . . . . . . . . . 61

4.1 Ansatz - Expansão de Galerkin . . . . . . . . . . . . . . . . . . . 61

4.2 Evolução Temporal das Amplitudes dos Modos e Condição de Ressonância

Triádica ............................. 62

4.3 Evolução Temporal para um Tripleto Ressonante . . . . . . . . . . . . . . 65

4.4 Determinação de um Tripleto Ressonante . . . . . . . . . . . . . . 67

4.5 Algumas soluções particulares para a evolução temporal de um tripleto . . 70

4.5.1 Estabilidade linear de apenas um modo . . . . . . . . . . . . . 70

4.5.2 Solução particular para amplitudes reais . . . . . . . . . . . . 71

4.6 Resultados Numéricos . . . . . . . . . . . . . . . . 75

5. Conclusões. . . . . . . . . . . . . . . . . . . . . 85

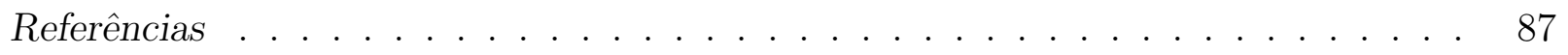


Capítulo 1

\section{Introdução}

A modelagem numérica da atmosfera sempre foi limitada pela capacidade computacional disponível. Em 1913 Lewis Fry Richardson foi o primeiro a utilizar um esquema de diferenças finitas para resolver as equações que descrevem a evolução temporal do estado da atmosfera. Entretanto, naquela época não haviam computadores eletrônicos, tornando a ideia de Richardson infactível (para mais informações sobre a evolução histórica da modelagem numérica da atmosfera veja Lynch (2008)). Mesmo com o advento do computador digital os primeiros modelos numéricos utilizavam grades grosseiras. Como o espaçamento da grade está diretamente relacionado com a escala espaço-temporal dos fenômenos que o modelo consegue resolver, é preciso simplificar as equações do movimento de acordo com as características da grade. O modelo das equações primitivas foi considerado por muito tempo o modelo mais simples que conseguia descrever os fenômenos de grande-escala na atmosfera. O crescente avanço tecnológico tem levado ao desenvolvimento de computadores com processadores cada vez mais velozes e com uma capacidade de memória e gráfica cada vez maior. Tal avanço computacional tem proporcionado a realização de simulações e/ou previsões numéricas com os modelos atmosféricos de previsão de tempo e clima com resoluções cada vez maiores. A utilização de modelos atmosféricos globais e com alta resolução tem sido uma das linhas de desenvolvimento da modelagem numérica da atmosfera, sendo tal procedimento atualmente factível, por exemplo, para a previsão climática sazonal. Uma das tendencias atuais da modelagem atmosférica é utilizar o mesmo modelo numérico tanto para a previsão do tempo quanto para previsões climáticas de longo prazo. O Met Office do Reino Unido busca esse tipo de unificação desde 1990 (Brown et al., 2012). O modelo unificado do Met Office já é utilizado tanto para previsão numérica do tempo com resolução de $1.5 \mathrm{~km}$ para fins operacionais e com resolução de centenas 
de metros para pesquisa, quanto para simulações climáticas de baixa resolução (grade de $300 \mathrm{~km}$ de resolução) com escalas de tempo variando de algumas horas até centenas de anos (Brown et al., 2012). A unificação dos modelos de previsão numérica do tempo e dos modelos climáticos possui várias vantagens, como a alteração e atualização desses modelos tornam-se mais econômica em relação a alteração e atualização de vários modelos para as diferentes escalas espaço-temporais, mas essa abordagem unificada também resulta em limitações para o modelo. Por exemplo, pequenos erros na conservação de massa de ar seco geralmente são insignificantes para a previsão regional de alguns dias, mas esse tipo de erro é extremamente prejudicial em simulações climáticas de centenas de anos Staniforth e Wood, 2006). A abordagem unificada também afeta as aproximações utilizadas no núcleo dinâmico do modelo. Por exemplo, a aproximação hidrostática é uma boa aproximação para os movimentos de escala planetária e sinótica, mas deixa de ser adequada para descrever fenômenos de mesoescala. Consequentemente, a utilização de modelos globais de altíssima resolução (com espaçamentos entre os pontos de grade menores que 10km) requer o uso de modelos atmosféricos não-hidrostáticos, uma vez que efeitos não-hidrostáticos tornam-se relevantes para modelos globais capazes de resolver estruturas atmosféricas com comprimentos de onda da ordem de 70km em latitudes médias (Daley, 1988). Para modelos oceânicos, a aproximação hidrostática deixa de ser válida para escalas horizontais entre 1 - 10km (Marshall et al., 1997). Portanto, entender a dinâmica de modelos globais não-hidrostáticos é uma questão de suma importância.

Para entender do ponto de vista teórico a dinâmica dos modelos atmosféricos globais e não-hidrostáticos, torna-se necessário analisar os modos normais desses modelos. Os modos normais representam os movimentos naturais de um sistema dinâmico e correspondem às soluções características das equações linearizadas, sem forçante, e em torno de um estado básico em repouso, constituindo assim as diferentes escalas que compõem a circulação atmosférica. O presente trabalho tem por objetivo analisar a energética dos modos normais de um modelo global não-hidrostático e também analisar as interações fracamente nãolineares desses modos normais. 


\subsection{Equações Aproximadas da Dinâmica dos Fluidos Geofísicos}

Durante muitos anos o sistema de equações conhecido como "equações primitivas" foi utilizado como núcleo dinâmico dos modelos numéricos globais de previsão do tempo. Neste modelo considera-se as equações de Navier-Stokes para um fluido invíscido em coordenadas esféricas e num referencial não-inercial em rotação uniforme (Gill, 1982). Além disso considera-se três aproximações: $(i)$ a aproximação hidrostática, $(i i)$ a aproximação de um fluido raso e a (iii) aproximação tradicional (White et al., 2005; Vallis, 2006). Na aproximação hidrostática os termos métricos, a componente vertical da força de Coriolis e as acelerações local e advectiva são desprezadas da componente vertical da equação da conservação do momento, resultando num balanço entre a força gravitacional e a componente vertical da força devido ao gradiente de pressão. Na aproximação de um fluido raso a coordenada radial $r$ é reescrita como $r \equiv a+z$, onde $a$ é o raio da Terra e $z$ é a altura local logo acima da superfície terrestre. Por fim, a aproximação tradicional despreza os termos métricos proporcionais à componente vertical do campo de vento e os termos da força de Coriolis proporcionais ao cosseno da latitude. As três aproximações empregadas no modelo das equações primitivas são apropriadas para sistemas rasos, ou seja, quando a escala horizontal característica for muito maior que a escala vertical característica (Pedlosky, 1987; Müller, 1989).

White et al. (2005) apresenta três modelos atmosféricos globais que resultam do enfraquecimento das hipóteses utilizadas no modelo das equações primitivas e, portanto, são mais adequados para a nova geração de modelos atmosféricos globais. Os três modelos ainda utilizam a aproximação de uma superfície geopotencial esférica. O primeiro modelo é o modelo não-hidrostático profundo onde aplica-se apenas a aproximação do geopotencial esférico nas equações de Navier-Stokes para um fluido invíscido, em coordenadas esferico-oblatas e num referencial não-inercial em rotação uniforme. Partindo do modelo não-hidrostático profundo pode-se obter o modelo quase-hidrostático, ou o modelo não-hidrostático raso. O modelo quase-hidrostático resulta da omissão do termo $d w / d t$ da componente vertical da equação do momento. O modelo não-hidrostático raso resulta da aplicação da aproximação de um fluido raso (i.e., aproximações (ii) e (iii)) no modelo não-hidrostático profundo. Por fim pode-se obter o modelo das equações primitivas ao aplicar a aproximação de um fluido raso no modelo quase-hidrostático ou omitindo o termo 


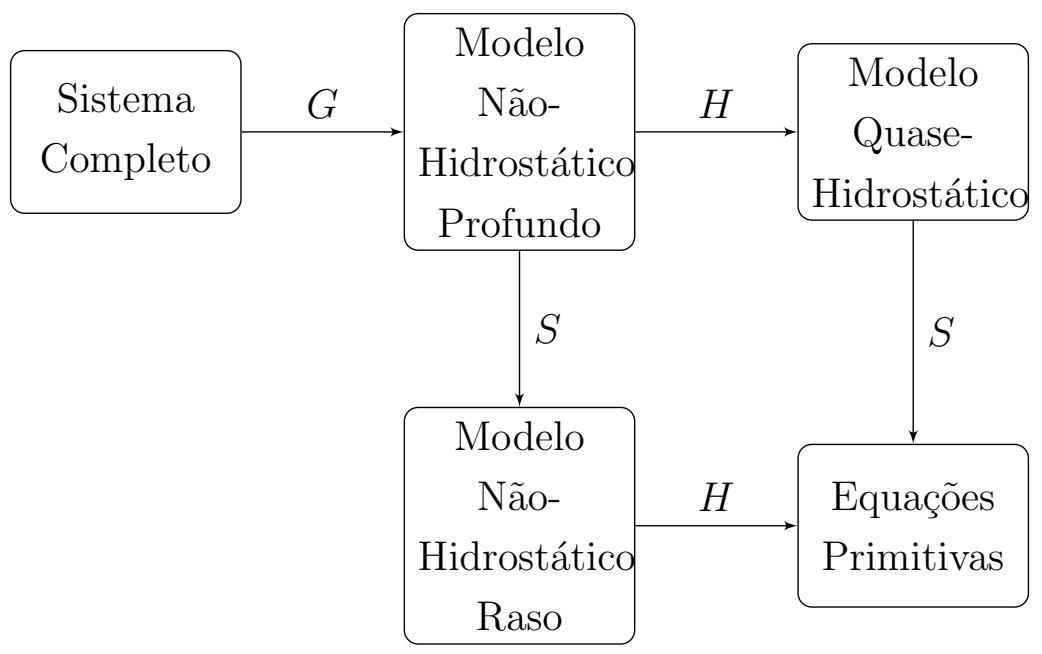

Figura 1.1: Diagrama representando a relação entre os modelos Não-Hidrostático Profundo, QuaseHidrostático, Não-Hidrostático Raso, Equações Primitivas Hidrostática e o modelo com as equações originais. As letras $G, H$ e $S$ indicam quais aproximações são utilizadas para obter um sistema de equações de outro sistema. O símbolo $G$ indica a aproximação do geopotencial esférico, $H$ indica a omissão do termo $d w / d t$ da componente vertical da equação do momento e $S$ indica as aproximações associadas a uma atmosfera rasa. Figura retirada de White et al. (2005).

$d w / d t$ da componente vertical da equação do momento do modelo não-hidrostático raso. A relação entre os modelos citados acima está representada de forma gráfica na Figura 1.1 . No presente trabalho será considerado o modelo não-hidrostático raso.

\subsection{Modelos Não-Hidrostáticos e Quase-Hidrostáticos}

É possível classificar os modelos utilizados em dinâmica de fluidos geofísicos em duas classes: modelos hidrostáticos e modelos não-hidrostáticos. A principal diferença entre essas duas classes está na presença de modos acústicos de altíssima frequência nos modelos não-hidrostáticos. Isso pode ser mostrado facilmente ao considerar um modelo bidimensional para as equações de Euler de um fluido adiabático (Pielke, 2002; Gill, 1982; Eckart, 1960). Num esquema numérico explícito, para garantir a estabilidade numérica do mesmo, o passo de tempo utilizado na integração do modelo deve ser menor que espaçamento da grade dividido pela velocidade do movimento mais rápido existente no modelo, isto é, o modelo deve satisfazer à condição de Courant-Friedrichs-Lewy (CFL). Como as ondas de som são as ondas não-eletromagnéticas mais rápidas presentes na atmosfera, a presença dessas ondas implica numa grande limitação no passo de tempo de integração do modelo. Além disso, acredita-se que os modos acústicos não são importantes para a maioria dos 
problemas encontrados em meteorologia de mesoescala (Pielke, 2002).

Devido à limitação imposta pela condição CFL muitos dos estudos relacionados aos modelos globais não-hidrostáticos tem como objetivo filtrar os modos acústicos ou contornar a limitação imposta pela condição CFL. Existem algumas formas de contornar esse problema: a utilização de filtros digitais, método da iniciação linear ou não-linear por modos normais, esquemas numéricos split-explicit ou ainda esquemas numéricos semi-implícitos (Daley, 1988; Murakami e Matsumura, 2007). Klemp et al. (2007) propõe um esquema numérico split-explicit conservativo para integrar modelos compressíveis e não-hidrostáticos; Dudhia e Bresch (2002) apresenta a versão global de um modelo numérico de mesoescala onde um esquema numérico split-explicit é utilizado para amortecer os modos acústicos de forma seletiva; Giraldo et al. (2010) apresentam várias formulações semi-implícitas da equação de Navier-Stokes com aplicações em modelos atmosféricos compressíveis e não-hidrostáticos; Klein (2009) mostra para quais limites assintóticos alguns modelos anelásticos são consistentes com o modelo totalmente compressível, de tal forma que esses modelos anelásticos possam ser úteis na construção de modelos atmosféricos robustos; Murakami e Matsumura (2007) propõem um novo método de iniciação não-linear por modos normais que visa diminuir a necessidade de armazenamento de dados para a utilização dos métodos convencionais de iniciação não-linear por modos normais. Existem muitos outros trabalhos relacionados à dinâmica atmosférica global não-hidrostática, mas a maioria desses trabalhos buscam a maneira mais eficiente de contornar a limitação gerada pelos modos acústicos.

Nos modelos hidrostáticos o termo da aceleração vertical $d w / d t$ é removido da componente vertical da equação da conservação do momento. Essa remoção do termo $d w / d t$ por sua vez remove (filtra) as ondas acústicas. Nos modelos não-hidrostáticos o termo $d w / d t$ é mantido e consequentemente os modelos não-hidrostáticos apresentam modos acústicos como uma das possíveis soluções características do modelo. Os modelos não-hidrostáticos podem ser classificados em elásticos e anelásticos. Os modelos elásticos correspondem aos modelos totalmente compressíveis, enquanto nos modelos anelásticos elimina-se o termo $\partial \rho / \partial t$ da equação da continuidade. Ao eliminar o termo $\partial \rho / \partial t$ surge a necessidade de modificar as equações do movimento e/ou da termodinâmica para que o sistema das equações governantes seja consistente. As diferentes modificações nas equações do movimento e da termodinâmica resultam em diferentes modelos anelásticos. Davies et al. (2003) discute as diferentes aproximações anelásticas e a validade das mesmas. A importância em classificar 
os modelos não-hidrostáticos em elásticos ou anelásticos está associado ao fato de que os modelos anelásticos filtram os modos acústicos que propagam-se na vertical e portanto elimina-se a limitação imposta pela condição CFL na presença dos modos acústicos.

Devido às limitações impostas pela condição CFL e por acreditar-se que os modos acústicos de altíssima frequência não tem importância significativa para descrever os processos dinâmicos da atmosfera, os últimos estudos relacionados aos modelos não-hidrostáticos procuram métodos de filtrar e/ou eliminar os modos acústicos.

A grande diferença entre o modelo das equações primitivas e o modelo quase-hidrostático está na presença dos termos da força de Coriolis proporcionais ao cosseno da latitude. Esses termos da força de Coriolis são chamados de termos de Coriolis Não-Tradicionais (NTC). A eliminação dos termos NTC na aproximação tradicional foi um dos pontos de maior discussão no modelo das equações primitivas. A eliminação dos termos NTC garante que o modelo das equações primitivas conserve momento angular e vorticidade potencial, além de garantir a separabilidade das estruturas horizontal e vertical das equações governantes linearizadas responsáveis pela característica dos modos normais do modelo (Thuburn et al. 2002a; Kasahara, 2004). Porém, os termos NTC podem ser importantes em regiões próximas ao equador (Bretherton, 1964) e para a dinâmica de mesoescala (Draghici, 1989). Ainda não se sabe exatamente qual o papel dinâmico dos termos NTC, mas recentemente vários estudos têm sido feitos nesse sentido (Kasahara e Gary, 2006, 2010; Fruman e Shepherd, 2008; Itano e Kasahara, 2011; Hayashi e Itano, 2012).

\subsubsection{Modos Normais}

Como mencionado anteriormente, para entender a dinâmica de um modelo é necessário conhecer os modos normais do mesmo. Além disso, no contexto dos fenômenos fracamente não-lineares, as soluções características das equações linearizadas em torno de um campo básico em repouso (i.e., os modos normais) podem ser utilizados como funções base para a expansão da solução do sistema mesmo no caso de estados básicos mais realistas, como no caso não-linear e também o caso com forçante. Este procedimento é o princípio do método de Galerkin (i.e., método espectral). Neste trabalho os modos normais serão utilizados para estudar os fenômenos fracamente não-lineares do modelo não-hidrostático raso. Mais especificamente os modos normais correspondem às oscilações de pequena amplitude do modelo em torno de um estado básico caracterizado por uma atmosfera 
em repouso, horizontalmente homogênea e estavelmente estratificada na vertical. Nessas condições, a hipótese do equilíbrio hidrostático para o estado básico é necessária para que o mesmo constitua uma solução de equilíbrio do sistema. Kasahara e Qian (2000) propuseram uma metodologia para obter os modos normais de um modelo atmosférico global não-hidrostático, compressível e baroclínico (i.e., o modelo não-hidrostático raso). Além disso, Kasahara e Qian (2000) analisaram a relação de dispersão dos modos normais do modelo não-hidrostático raso. O presente trabalho tem por objetivo estender o estudo de Kasahara e Qian (2000), analisando a energética linear e fracamente não-linear dos modos normais do modelo não-hidrostático raso. Entre as interações fracamente não-lineares a interação entre tripletos ressonantes foi utilizada para estudar como ondas tropicalmente confinadas podem excitar onda de Rossby barotrópicas em médias latitudes (Raupp et al. 2008; Khouider et al., 2013).

Nos modelos globais não-hidrostáticos os termos NTC não afetam de forma significativa os modos gravito-inerciais e os modos de Rossby-Hauwritz, porém afetam de forma significativa a estrutura dos modos acústico-inerciais presentes no modelo não-hidrostático profundo (Thuburn et al., 2002a). Além disso, os termos NTC, ao utilizar-se condições de contorno do tipo tampa rígida na vertical, dão origem a um novo modo normal de oscilação, o Boundary-Induced Inertial Mode (BII) Kasahara, 2003b a; Thuburn et al., 2002b).

A utilização de modelos não-hidrostáticos implica no aparecimento de modos acústicos entre as soluções características do sistema. Além disso, nos modelos globais não-hidrostáticos, a frequência dos modos gravito-inerciais passam a ser limitados superiormente pela frequência de Brunt-Väisälä e, portanto, os modos gravito-inerciais mais curtos são afetados pelos efeitos não-hidrostáticos (Daley, 1988; Kasahara e Qian, 2000). É importante ressaltar que ambos os modelos não-hidrostáticos raso e profundo apresentam os modos acústicos como solução característica do sistema, entretanto a versão rasa é o modelo global nãohidrostático mais simples que permite a existência dos modos acústicos. Por esse motivo o modelo não-hidrostático raso será o modelo utilizado para o estudo aqui desenvolvido. 


\subsection{Objetivos e Organização do Texto}

Este trabalho tem por objetivo analisar a energética dos modos normais de um modelo global não-hidrostático e as interações fracamente não-lineares entre os modos normais exclusivos dos modelos não-hidrostáticos. Uma das principais características dos modelos não-hidrostáticos está na presença dos modos acústicos como uma das soluções características. Esses modos acústicos, em geral, são considerados como ruídos (i.e., fisicamente irrelevantes) e devido à limitação no passo de tempo de integração imposta pela condição de CFL na presença desses modos, os mesmos costumam ser filtrados e/ou eliminados dos modelos. No presente trabalho também será analisado as interações não-lineares entre os modos normais presentes no modelo. Mais especificamente, serão analisadas as trocas de energia num tripleto ressonante formado por dois modos acústicos e um modo de gravidade.

Primeiramente, no capítulo 2 as equações que representam o modelo não-hidrostático raso são apresentadas. E assumindo que as variáveis dinâmicas podem ser escritas como a soma de um campo básico mais uma perturbação em relação ao campo básico, obtém-se as equações governantes do problema abordado. No capítulo 3 os modos normais do mesmo são determinados, seguindo o procedimento proposto por Kasahara e Qian (2000), e em seguida, será derivada a partir das equações governantes na forma Euleriana a energia total referente às perturbações e, a partir da decomposição modal desta energia (via teorema de Parseval), será analisada a energética dos diferentes modos de oscilação presentes no modelo. No capítulo 4, utilizando um método perturbativo determina-se as equações dinâmicas que descrevem as interações fracamente não-lineares do sistema. Posteriormente essas equações dinâmicas são simplificadas para o caso das interações não-lineares de apenas três modos normais. Partindo da relação de dispersão dos modos normais do modelo determina-se um tripleto ressonante composto por dois modos acústicos e um modo de gravidade. As equações que descrevem a evolução temporal da amplitude dos modos que compõem um tripleto ressonante são resolvidas e é feito uma análise das trocas de energia entre os modos que compõe o tripleto. Por fim, no capítulo 5 são apresentados as conclusões do trabalho e sugestões para trabalhos futuros. 


\section{Capítulo 2}

\section{Equações Governantes e Teoria da Perturbação}

O modelo descrito em Kasahara e Qian (2000) é global, não-hidrostático, compressível e baroclínico. Para escrever as equações do movimento, não será utilizado um sistema de coordenadas esféricas convencional, mas um sistema de coordenadas esférico oblato, na forma de um elipsoide, discutido no livro de Gill (1982). Nesse sistema de coordenadas temos $\phi$ como a latitude, $\lambda$ como a longitude e $z$ como a altitude relativa ao raio da Terra $a$, suposto constante. Neste contexto, as equações da conservação do momento linear são dadas por:

$$
\begin{aligned}
\frac{d u}{d t}-\left(2 \Omega+\frac{u}{a \cos \phi}\right)(v \sin \phi-w \cos \phi) & =-\frac{1}{\rho a \cos \phi} \frac{\partial p}{\partial \lambda}+F_{\lambda} \\
\frac{d v}{d t}+\frac{w v}{a}+\left(2 \Omega+\frac{u}{a \cos \phi}\right) u \sin \phi & =-\frac{1}{\rho a} \frac{\partial p}{\partial \phi}+F_{\phi} \\
\frac{d w}{d t}-\frac{v^{2}}{a}-\left(2 \Omega+\frac{u}{a \cos \phi}\right) u \cos \phi & =-\frac{1}{\rho} \frac{\partial p}{\partial z}-g+F_{r}
\end{aligned}
$$

onde $u, v, w$, são as componentes zonal, meridional e vertical do campo de velocidade, respectivamente, $\Omega$ é a velocidade angular da Terra, $\rho$ é a densidade do ar e $F_{\phi}, F_{\lambda}, F_{r}$ são as componentes meridional, zonal e radial da força viscosa, $g$ é a aceleração da gravidade, considerada constante, e $p$ é a pressão. Note que $d / d t$ é a derivada total, que será definida posteriormente.

Para obter as equações utilizadas no modelo, considera-se um fluido invíscido e, além disso, via análise de escala, desconsidera-se os termos devido a curvatura do sistema de 
coordenadas e alguns termos associados à aceleração de Coriolis, obtendo:

$$
\begin{aligned}
\frac{d u}{d t}-\left(f+\frac{u \tan \phi}{a}\right) v & =-\frac{1}{\rho a \cos \phi} \frac{\partial p}{\partial \lambda} \\
\frac{d v}{d t}+\left(f+\frac{u \tan \phi}{a}\right) u & =-\frac{1}{\rho a} \frac{\partial p}{\partial \phi} \\
\delta_{H} \frac{d w}{d t} & =-\frac{1}{\rho} \frac{\partial p}{\partial z}-g
\end{aligned}
$$

onde $\delta_{H}$ é igual a 1 para o caso não-hidrostático e 0 para o caso hidrostático.

Para fechar o sistema de equações, consideramos também a equação da continuidade, a equação de estado do gás ideal e a equação da termodinâmica para movimentos adiabáticos:

$$
\begin{aligned}
\frac{d \rho}{d t}+\rho\left(\vec{\nabla} \cdot \vec{V}+\frac{\partial w}{\partial z}\right) & =0 \\
p & =\rho R T \\
\frac{d p}{d t} & =\gamma R T \frac{d \rho}{d t}
\end{aligned}
$$

onde $T$ é a temperatura, $R$ é a constante dos gases para o ar seco, $\gamma=C_{p} / C_{v}$, onde $C_{p}$ é o calor específico para pressão constante e $C_{v}$ é o calor específico para volume constante. Os operadores diferenciais utilizados nas equações acima são definidos por:

$$
\begin{aligned}
\frac{d}{d t} & =\frac{\partial}{\partial t}+\vec{V} \cdot \vec{\nabla}+w \frac{\partial}{\partial z} \\
\vec{V} \cdot \vec{\nabla} & =\frac{u}{a \cos \phi} \frac{\partial}{\partial \lambda}+\frac{v}{a} \frac{\partial}{\partial \phi} \\
\vec{\nabla} \cdot \vec{V} & =\frac{1}{a \cos \phi}\left(\frac{\partial u}{\partial \lambda}+\frac{\partial}{\partial \phi}(v \cos \phi)\right)
\end{aligned}
$$

Em (2.4)-(2.12), $\vec{V}=(u, v)$ representa o vetor vento horizontal e $f=2 \Omega \sin \phi$ refere-se ao parâmetro de Coriolis. O sistema de equações (2.4)-(2.9) é a versão não-hidrostática do modelo das equações primitivas e é conhecido como modelo não hidrostático raso White et al. (2005).

Para poder analisar a energética dos modos normais do modelo não hidrostático raso vamos assumir que as variáveis dinâmicas podem ser escritas como uma perturbação sobreposta a um estado básico: 


$$
\begin{aligned}
& u(\phi, \lambda, z, t)=u_{0}+u^{\prime}(\phi, \lambda, z, t) \\
& v(\phi, \lambda, z, t)=v_{0}+v^{\prime}(\phi, \lambda, z, t) \\
& w(\phi, \lambda, z, t)=w_{0}+w^{\prime}(\phi, \lambda, z, t) \\
& p(\phi, \lambda, z, t)=p_{0}(z)+p^{\prime}(\phi, \lambda, z, t) \\
& \rho(\phi, \lambda, z, t)=\rho_{0}(z)+\rho^{\prime}(\phi, \lambda, z, t) \\
& T(\phi, \lambda, z, t)=T_{0}+T^{\prime}(\phi, \lambda, z, t)
\end{aligned}
$$

supondo que o estado básico é isotérmico, hidrostático e está em repouso, ou seja:

$$
u_{0}=v_{0}=w_{0}=0, p_{0}=p_{0}(z), \rho_{0}=\rho_{0}(z), T_{0}=\text { const }
$$

Em 2.19), os perfis de $p_{0}(z)$ e $\rho_{0}(z)$ estão em equilíbrio hidrostático, ou seja:

$$
\frac{d p_{0}}{d z}=-\rho_{0} g
$$

Substituindo (2.13)-(2.20) nas equações (2.4)- 2.9 e retendo somente os termos de até segunda ordem em termos das perturbações, obtém-se:

$$
\begin{gathered}
\frac{\partial u^{\prime}}{\partial t}-f v^{\prime}+\frac{1}{a \rho_{0} \cos \phi} \frac{\partial p^{\prime}}{\partial \lambda}=-\vec{V}^{\prime} \cdot \nabla u^{\prime}-w^{\prime} \frac{\partial u^{\prime}}{\partial z}+\frac{u^{\prime} v^{\prime}}{a} \tan \phi+\frac{\rho^{\prime}}{a \rho_{0}^{2} \cos \phi} \frac{\partial p^{\prime}}{\partial \lambda} \\
\frac{\partial v^{\prime}}{\partial t}+f u^{\prime}+\frac{1}{a \rho_{0}} \frac{\partial p^{\prime}}{\partial \phi}=-\vec{V}^{\prime} \cdot \nabla v^{\prime}-w^{\prime} \frac{\partial v^{\prime}}{\partial z}-\frac{u^{\prime} u^{\prime}}{a} \tan \phi+\frac{\rho^{\prime}}{a \rho_{0}^{2}} \frac{\partial p^{\prime}}{\partial \phi} \\
\frac{\partial w^{\prime}}{\partial t}+\frac{1}{\rho_{0}} \frac{\partial p^{\prime}}{\partial z}+\frac{g}{C_{s}^{2} \rho_{0}} p^{\prime}-\frac{\theta^{\prime}}{\rho_{0}}=-\vec{V}^{\prime} \cdot \nabla w^{\prime}-w^{\prime} \frac{\partial w^{\prime}}{\partial z}+\frac{\rho^{\prime}}{\rho_{0}^{2}} \frac{\partial p^{\prime}}{\partial z}+g \frac{\rho^{\prime 2}}{\rho_{0}^{2}} \\
\frac{1}{C_{s}^{2}} \frac{\partial p^{\prime}}{\partial t}-\frac{g \rho_{0}}{C_{s}^{2}} w^{\prime}+\rho_{0}\left(\nabla \cdot \vec{V}^{\prime}+\frac{\partial w^{\prime}}{\partial z}\right)=-\frac{1}{C_{s}^{2}}\left(\vec{V}^{\prime} \cdot \nabla p^{\prime}+w^{\prime} \frac{\partial p^{\prime}}{\partial z}\right)+ \\
+\frac{1}{C_{s}^{2}} \frac{T^{\prime}}{T_{0}}\left(-g \rho_{0} w^{\prime}+\frac{\partial p^{\prime}}{\partial t}\right)-\rho^{\prime}\left(\nabla \cdot \vec{V}^{\prime}+\frac{\partial w^{\prime}}{\partial z}\right) \\
\frac{\partial \theta^{\prime}}{\partial t}+N^{2} w^{\prime} \rho_{0}=-\vec{V}^{\prime} \cdot \nabla \theta^{\prime}-w^{\prime} \frac{\partial \theta^{\prime}}{\partial z}+\frac{g}{C_{s}^{2}} \frac{T^{\prime}}{T_{0}}\left(-g \rho_{0} w^{\prime}+\frac{\partial p^{\prime}}{\partial t}\right)
\end{gathered}
$$

onde $N$ é a frequência de Brunt-Väisälä, $C_{s}$ a velocidade de propagação das ondas sonoras puras, $\nabla \cdot \vec{V}^{\prime}$ o divergente da perturbação do campo de vento horizontal e $\theta^{\prime}$ representa 
uma mudança de variável conveniente, sendo dados por:

$$
\begin{aligned}
N^{2} & =-g\left(\frac{1}{\rho_{0}} \frac{d \rho_{0}}{d z}+\frac{g}{C_{s}^{2}}\right)=\frac{g \kappa}{H} \\
C_{s}^{2} & =\gamma R T_{0} \\
\nabla \cdot \vec{V}^{\prime} & =\frac{1}{a \cos \phi}\left(\frac{\partial u^{\prime}}{\partial \lambda}+\frac{\partial}{\partial \phi}\left(v^{\prime} \cos \phi\right)\right) \\
\theta^{\prime} & =\frac{g}{C_{s}^{2}} p^{\prime}-g \rho^{\prime}
\end{aligned}
$$

onde $H=R T_{0} / g$ é a escala de altura de uma atmosfera isotérmica e $\kappa=R / C_{p}$.

A frequência de Brunt-Väisälä está associada ao movimento oscilatório de uma parcela de fluido, quando a mesma, estando estaticamente estável, é deslocada do seu ponto de equilíbrio, o que resulta no surgimento de uma força restauradora, proporcional ao deslocamento da parcela.

Kasahara e Qian (2000) utilizaram a mesma teoria de perturbação mas retendo somente os termos de primeira ordem (lineares) em relação às perturbações. Logo, o estudo aqui realizado visa estender a teoria de Kasahara e Qian (2000) para os modos normais não hidrostáticos de modo a incluir os termos não lineares de mais baixa ordem (quadráticos).

O sistema (2.21)- 2.25 pode ser reescrito da seguinte forma:

$$
\mathcal{L} \vec{u}=\mathcal{N}(\vec{u}, \vec{u})
$$

onde $\vec{u}:=\left[u^{\prime}, v^{\prime}, w^{\prime}, p^{\prime}, \theta^{\prime}\right]^{T}$ é um vetor com as variáveis dinâmicas, $\mathcal{N}$ é um vetor com os termos não-lineares quadráticos:

$$
\mathcal{N}(\vec{u}, \vec{u})=\left[\begin{array}{c}
-\vec{V}^{\prime} \cdot \nabla u^{\prime}-w^{\prime} \frac{\partial u^{\prime}}{\partial z}+\frac{u^{\prime} v^{\prime}}{a} \tan \phi+\frac{\rho^{\prime}}{a \rho_{0}^{2} \cos \phi} \frac{\partial p^{\prime}}{\partial \lambda} \\
-\vec{V}^{\prime} \cdot \nabla v^{\prime}-w^{\prime} \frac{\partial v^{\prime}}{\partial z}-\frac{u^{\prime} u^{\prime}}{a} \tan \phi+\frac{\rho^{\prime}}{a \rho_{0}^{2}} \frac{\partial p^{\prime}}{\partial \phi} \\
-\vec{V}^{\prime} \cdot \nabla w^{\prime}-w^{\prime} \frac{\partial w^{\prime}}{\partial z}+\frac{\rho^{\prime}}{\rho_{0}^{2}} \frac{\partial p^{\prime}}{\partial z}+g \frac{\rho^{\prime 2}}{\rho_{0}^{2}} \\
-\frac{1}{C_{s}^{2}}\left(\vec{V}^{\prime} \cdot \nabla p^{\prime}+w^{\prime} \frac{\partial p^{\prime}}{\partial z}\right)+\frac{1}{C_{s}^{2}} \frac{T^{\prime}}{T_{0}}\left(-g \rho_{0} w^{\prime}+\frac{\partial p^{\prime}}{\partial t}\right)-\rho^{\prime}\left(\nabla \cdot \overrightarrow{V^{\prime}}+\frac{\partial w^{\prime}}{\partial z}\right) \\
-\vec{V}^{\prime} \cdot \nabla \theta^{\prime}-w^{\prime} \frac{\partial \theta^{\prime}}{\partial z}+\frac{g}{C_{s}^{2}} \frac{T^{\prime}}{T_{0}}\left(-g \rho_{0} w^{\prime}+\frac{\partial p^{\prime}}{\partial t}\right)
\end{array}\right]
$$

e $\mathcal{L}$ é um operador diferencial linear definido por: 


$$
\mathcal{L}=\left[\begin{array}{ccccc}
\frac{\partial}{\partial t} & -f & 0 & \frac{1}{a \rho_{0} \cos \phi} \frac{\partial}{\partial \lambda} & 0 \\
f & \frac{\partial}{\partial t} & 0 & \frac{1}{a \rho_{0}} \frac{\partial}{\partial \phi} & 0 \\
0 & 0 & \frac{\partial}{\partial t} & \frac{1}{\rho_{0}}\left(\frac{\partial}{\partial z}+\frac{g}{C_{s}^{2}}\right) & -\frac{1}{\rho_{0}} \\
\frac{\rho_{0}}{a \cos \phi} \frac{\partial}{\partial \lambda} & \frac{\rho_{0}}{a \cos \phi}\left(\cos \phi \frac{\partial}{\partial \phi}-\sin \phi\right) & \rho_{0}\left(\frac{\partial}{\partial z}-\frac{g}{C_{s}^{2}}\right) & \frac{1}{C_{s}^{2}} \frac{\partial}{\partial t} & 0 \\
0 & 0 & \rho_{0} N^{2} & 0 & \frac{\partial}{\partial t}
\end{array}\right]
$$


Capítulo 3

\section{Modos Normais do Sistema Linearizado}

\subsection{Modos Normais do Sistema Linearizado}

Para estudar as interações fracamente não-lineares permitidas pelo modelo 2.30 utilizaremos uma expansão de Galerkin para representar as variáveis dinâmicas. Neste caso utilizaremos como funções base as autofunções da versão linearizada de 2.30) ( i.e., as soluções características de 2.30 quando $\mathcal{N}=0$ ), ou seja:

$$
\mathcal{L} \vec{u}=0
$$

Kasahara e Qian (2000) resolveram o problema (3.1) para condições de fronteira do tipo tampa rígida na vertical, ou seja, $w^{\prime}=0$ em $z=0$ e $z=z_{T}$ e supondo regularidade da solução no polos. Assim, nesta seção do presente capítulo mostraremos em detalhe o desenvolvimento teórico bem como o esquema numérico propostos por Kasahara e Qian (2000) para obter os modos normais do sistema (3.1). De acordo com Kasahara e Qian (2000), uma separação de variáveis conveniente para obter as soluções características do sistema (3.1) é dada por:

$$
\left[\begin{array}{c}
\rho_{0}^{1 / 2} u^{\prime} \\
\rho_{0}^{1 / 2} v^{\prime} \\
\rho_{0}^{-1 / 2} p^{\prime} \\
\rho_{0}^{1 / 2} w^{\prime} \\
\rho_{0}^{-1 / 2} \theta^{\prime}
\end{array}\right]=\left[\begin{array}{c}
U(\phi) \xi(z) \\
i V(\phi) \xi(z) \\
P(\phi) \xi(z) \\
i P(\phi) \eta(z) \\
P(\phi) \theta(z)
\end{array}\right] \exp [i(s \lambda-\sigma t)]
$$

onde $i$ representa a unidade imaginária, $s$ é o número de onda zonal e $\sigma$ é a frequência temporal de oscilação dos modos. Substituindo (3.2) na equação (3.1), obtemos o seguinte 
problema de autovalor:

$$
\begin{aligned}
-\sigma U-f V+\frac{s P}{a \cos \phi} & =0 \\
f U+\sigma V+\frac{1}{a} \frac{d P}{d \phi} & =0 \\
\delta_{H} \sigma \eta+\frac{d \xi}{d z}+\Gamma \xi-\theta & =0 \\
-\sigma \theta+N^{2} \eta & =0 \\
-\frac{\sigma}{C_{s}^{2}} \xi+\frac{1}{a \cos \phi}\left[s U+\frac{d}{d \phi}(V \cos \phi)\right] \xi P^{-1}+\frac{d \eta}{d z}-\left(\frac{1}{2 \rho_{0}} \frac{d \rho_{0}}{d z}+\frac{g}{C_{s}^{2}}\right) \eta & =0
\end{aligned}
$$

onde o parâmetro $\Gamma$ é definido posteriormente em (3.15). As equações (3.3) e (3.4) contém termos que dependem apenas de $\phi$ e nenhum termo que depende de $z$. Por outro lado, as equações 3.5) e (3.6) possuem termos que dependem apenas de $z$. Na equação 3.7) apenas o segundo termo depende de $\phi$, enquanto os outros termos são funções de $z$. No intuito de excluir essa depêndencia em $\phi$ dessa equação, introduz-se o parâmetro de separação $h_{e}$.

A partir das equações (3.3) e (3.4), é possível obter uma expressão para $U$ e para $V$ :

$$
\begin{gathered}
U=\frac{1}{\left(\sigma^{2}-f^{2}\right)}\left(\frac{\sigma s P}{a \cos \phi}+\frac{f}{a} \frac{d P}{d \phi}\right) \\
V=-\frac{1}{\sigma^{2}-f^{2}}\left(\frac{\sigma}{a} \frac{d P}{d \phi}+\frac{2 \Omega s \sin \phi P}{a \cos \phi}\right)
\end{gathered}
$$

Com o auxílio de (3.8) e (3.9), o termo da divergência horizontal em (3.7) pode ser reescrito apenas em função de $P$, i.e.,

$$
\frac{1}{a \cos \phi}\left[s U+\frac{d}{d \phi}(V \cos \phi)\right] \equiv \sigma H_{L}(P)
$$

com o operador $H_{L}$ sendo escrito como:

$$
\begin{aligned}
H_{L}:= & \sigma\left[\frac{s}{\sigma a \cos \phi\left(\sigma^{2}-f^{2}\right)}\left(\frac{s \sigma}{a \cos \phi}+\frac{2 \Omega \sin \phi}{a} \frac{d}{d \phi}\right)\right] \\
& -\sigma\left[\frac{1}{\sigma a \cos \phi} \frac{d}{d \phi}\left(\frac{1}{\sigma^{2}-f^{2}}\left(\frac{2 \Omega \sin \phi}{a} s+\frac{\sigma \cos \phi}{a} \frac{d}{d \phi}\right)\right)\right]
\end{aligned}
$$

O operador $H_{L}$ é conhecido como operador da maré de Laplace.

Dada a dependência em $\phi$ da equação $(3.7)$, define-se o parâmetro de separação $h_{e}$ :

$$
H_{L}(P)=\frac{1}{g h_{e}} P
$$


A equação (3.12) refere-se à condição para que existam soluções separáveis em $\phi$ e $z$ ou à condição de separabilidade da solução. O parâmetro de separação $h_{e}$ é também conhecido como altura equivalente e foi introduzida por Taylor (1936), no contexto hidrostático.

Utilizando 3.12 e 3.11) é possível reescrever 3.7) como:

$$
\sigma \xi\left(\frac{1}{C_{s}^{2}}-\frac{1}{g h_{e}}\right)=L_{1}(\eta)
$$

onde o operador $L_{1}$ é dado por

$$
L_{1}:=\frac{d}{d z}-\Gamma
$$

Em (3.14), o parâmetro $\Gamma$ é definido como,

$$
\Gamma=\frac{1}{2 \rho_{0}} \frac{d \rho_{0}}{d z}+\frac{g}{C_{s}^{2}}=\frac{1}{2}\left(\frac{g}{C_{s}^{2}}-\frac{N^{2}}{g}\right)=\frac{1-2 \kappa}{H}
$$

sendo $H=R T_{0} / g$. O parâmetro $\Gamma$ é importante na teoria das oscilações em fluidos geofísicos, como no problema do ajuste para o equilíbrio, na presença da força gravitacional, de um fluido compressível e estratificado (Gill, 1982). O parâmetro $\Gamma$ também está associado ao processo de expansão adiabática (Eckart, 1960).

Vale notar que as equações (3.5), (3.6) e (3.13) descrevem a estrutura vertical dos modos normais, enquanto as equações (3.12), (3.8) e (3.9) fornecem a estrutura horizontal dos mesmos. Portanto, o problema resume-se em resolver as equações (3.5), (3.6) e (3.13) para obter as funções $\theta, \xi$ e $\eta$. Com a estrutura vertical resolvida, o próximo passo é resolver (3.12) com $P$ sendo a autofunção e por fim determinar $U$ e $V$ por (3.8) e (3.9), respectivamente. Assim, com o intuito de resolver o problema de autovalor para a estrutura vertical dos modos, eliminando $\theta$ e $\xi$ das equações (3.5), (3.6) e (3.14) chega-se em

$$
\frac{d^{2} \eta}{d z^{2}}+\left(\lambda-\Gamma^{2}\right) \eta=0
$$

onde

$$
\lambda=\left(\frac{1}{g h_{e}}-\frac{1}{C_{s}^{2}}\right)\left(N^{2}-\delta_{H} \sigma^{2}\right)
$$

A equação (3.16) é a equação da estrutura vertical, no caso isotérmico, que governa a dependência de $\rho_{0}^{1 / 2} w^{\prime}$ com a altura $z$. A solução de (3.16), com condições de contorno do 
tipo tampa rígida ("rigid lid"), dadas por (3.25), é dada por:

$$
\eta(z)=A_{k} \sin \hat{k} z
$$

desde que seja satisfeita a relação:

$$
\hat{k}^{2}+\Gamma^{2}=\lambda_{k}
$$

onde $A_{k}$ é uma constante arbitrária, $\hat{k}=k \pi / z_{T}$, e $k=1,2, \ldots$ Substituindo (3.18) em (3.13), segue que:

$$
\xi(z)=\left(\frac{1}{C_{s}^{2}}-\frac{1}{g h_{e}}\right)^{-1} \frac{A_{k}}{\sigma}(\hat{k} \cos \hat{k} z-\Gamma \sin \hat{k} z)
$$

Com $\eta$ e $\xi$ determinados pode-se determinar $\theta(z)$ através de 3.6

$$
\theta(z)=\frac{N^{2}}{\sigma} \eta
$$

Uma solução especial de (3.3)-(3.7), não incluída em (3.18)-(3.21), ocorre quando não há movimentos verticais, ou seja $w^{\prime} \equiv 0$. Nesse caso, devido à separação de variáveis utilizada, também teremos que $\eta(z) \equiv 0$. De 3.13 , segue que esta solução especial é caracterizada por um parâmetro de separação $h_{e}$ dado por:

$$
h_{e x}=\frac{C_{s}^{2}}{g}=\frac{H}{1-\kappa}=\frac{7}{5} H
$$

No caso da atmosfera Terrestre, $h_{e x}$ vale aproximadamente $10 \mathrm{~km}$. Vale notar que, como $w^{\prime}=0$, essa solução também existe na aproximação hidrostática. De (3.22) e (3.17), verifica-se que esta solução está associada com o autovalor $\lambda=0$. Considerando $\eta=0$ e as equações (3.5) e (3.6), segue que a estrutura vertical das flutuações do vento horizontal e da pressão associadas a este modo é dada por:

$$
\xi(z)=\xi_{0} e^{-\Gamma z}
$$

onde $\xi_{0}$ é uma constante arbitrária. Com essa estrutura vertical, a estrutura horizontal será composta apenas por oscilações de primeira e segunda espécies, pois os movimentos verticais são identicamente nulos e essa solução também existe no caso hidrostático, quando $\delta_{H}=0$, onde não existem modos acústico-inerciais (i.e., oscilações de terceira espécie) na 
estrutura horizontal. Portanto, para este modo vertical caracterizado por $h_{e}=h_{e x}$, o movimento horizontal é composto apenas por oscilações de primeira e segunda espécies. Neste caso, as oscilações de primeira espécie, ondas gravito-inerciais de leste e de oeste, são chamadas de ondas de Lamb (Gill, 1982), pois Lamb estudou a propagação de ondas numa atmosfera isotérmica, compressível, sem rotação e com movimentos adiabáticos. Porém, no presente modelo oscilações de segunda espécie (ondas de Rossby-Haurwitz) também existem no contexto do presente trabalho porque o modelo proposto é (além de isotérmico, adiabático e compressível) global e está em rotação. Então, para o autovalor $\lambda=0$ da estrutura vertical, daqui em diante denominaremos esse último modo de modo externo enquanto as oscilações de primeira espécie associadas a este modo vertical serão denominadas ondas de Lamb.

\subsubsection{Problema de autovalor}

O sistema de equações (3.3)-(3.7), com condições de contorno apropriadas, resultam em dois problemas de autovalor para as estruturas vertical e horizontal. Para o caso hidrostático, o parâmetro de separação $h_{e}$ é obtido resolvendo apenas estrutura vertical e uma vez que o valor de $h_{e}$ é fixado, é possível determinar $\sigma$ resolvendo a estrutura horizontal.

No caso não-hisdrostático, porém, a altura equivalente $h_{e}$ e a frequência $\sigma$ estão presentes tanto no problema da estrutura vertical como no problema da estrutura horizontal e, portanto, os dois problemas devem ser resolvidos simultaneamente como um sistema de equações acopladas. Como a estrutura horizontal só pode ser resolvida numericamente, um método iterativo, proposto por Kasahara e Qian (2000), é utilizado para determinar $\sigma$ e $h_{e}$.

Para resolver o problema da estrutura horizontal, impõe-se que $P(\phi)$ seja uma função regular nos polos, ou seja:

$$
P=0 \text { em } \phi=\frac{\pi}{2} \text { e } \phi=-\frac{\pi}{2}
$$

Dessa forma, especificando-se um valor para $h_{e}$, é possível determinar a autofunção $P(\phi)$ e o autovalor $\sigma$ da equação (3.10). O método de obtenção e as propriedades das autofunções da estrutura horizontal, ou seja, as autofunções do operador da maré de Laplace, já foi discutida de forma extensiva por Hough (1898); Longuet-Higgins (1968); Kasahara (1976, 
1977, 1978); Chapman e Lindzen (1970); Swarztrauber e Kasahara (1985); Bonatti et al. (1983); Bonatti e Silva Dias (1983), entre outros. Bonatti et al. (1983) calculam as funções de Hough a partir de um modelo de equações primitivas e analisam as características dispersivas dos modos, a questão do reforço e a aplicabilidade desses modos na previsão do tempo. Bonatti e Silva Dias (1983) descrevem um modelo espectral barotrópico não-linear e global de equações primitivas, onde as funções de Hough são utilizadas como funções base do modelo espectral. Além disso, Bonatti e Silva Dias (1983) discutem, baseados na estabilidade numérica e dispersão dos modos, qual esquema numérico deve ser utilizado para a integração da amplitude dos modos normais utilizados na expansão espectral e comparam os esquemas de inicialização linear e não-linear por modos normais no modelo considerado.

Para o problema da estrutura vertical, é comum impor que $w^{\prime}=0$ em $z=0$, como condição de contorno na região inferior. Como condição de contorno superior, geralmente é imposto que a energia cinética das perturbações seja limitada quando $z \rightarrow \infty$ Kasahara e Qian, 2000). Mas como o objetivo deste estudo são os modos normais, impõe-se uma condição de fronteira rígida, tanto na superfície como no topo da atmosfera. Assim, a condição de contorno vertical é dada por:

$$
\eta=0 \text { em } z=0 \text { e } z=z_{T}
$$

onde $z_{T}$ é a altura do topo hipotético da atmosfera.

\subsubsection{Solução da estrutura horizontal(Funções de Hough)}

As equações que compõe o problema de autovalor e autofunção e as condições de contorno para a estrutura horizontal são dadas por:

$$
\begin{array}{r}
-\sigma U-f V+\frac{s P}{a \cos \phi}=0 \\
f U+\sigma V+\frac{1}{a} \frac{d P}{d \phi}=0 \\
H_{L}(P)=\frac{1}{g h_{e}} P \\
P=0 \text { em } \phi=\frac{\pi}{2} \text { e } \phi=-\frac{\pi}{2}
\end{array}
$$

e a solução desse problema é apresentada em detalhes no artigo de Longuet-Higgins (1968). Entretanto, nesse artigo foi utilizado um sistema de coordenadas esférico, diferente do sistema de coordenadas proposto neste trabalho. Consequentemente, nesta seção da presente 
dissertação o problema de autovalor referente à equação da maré de Laplace descrito acima será abordado novamente, seguindo basicamente a mesma metodologia de Longuet-Higgins (1968) mas adaptada ao sistema de coordenadas utilizado no presente trabalho. Tal procedimento aqui abordado corresponde àquele adotado por Kasahara (1976, 1977, 1978). Logo, será descrito neste item da presente seção a metodologia apresentada por Kasahara (1976, 1977, 1978) para obter as autosoluções da equação da maré de Laplace. Além disso, devido ao ansatz da solução dado por (3.2), será considerado a seguinte substituição $\partial / \partial \lambda \rightarrow i s$.

Primeiramente serão deduzidas algumas identidades que auxiliarão na solução do problema. Por exemplo, o laplaciano no sistema de coordenadas aqui utilizado é definido como (Gill, 1982):

$$
\vec{\nabla}^{2} \equiv\left[\frac{-s^{2}}{a^{2} \cos \phi^{2}}+\frac{1}{a^{2} \cos \phi^{2}} \frac{\partial}{\partial \phi}\left(\cos \phi \frac{\partial}{\partial \phi}\right)\right]
$$

Pela identidade de Helmholtz, o campo de velocidade $\vec{V}=(u, v)$ pode ser decomposto por uma soma de dois campos vetoriais, um irrotacional dado por $\vec{\nabla} \Phi$ e outro solenoidal representado por $\vec{\nabla} \times \vec{\Psi}=\vec{\nabla} \times(0,0, \psi)=\hat{k} \times \nabla \psi$ :

$$
\begin{aligned}
\vec{V} & =\vec{\nabla} \Phi+\hat{k} \times \nabla \psi \\
& =\left(\frac{1}{\cos \phi} \frac{\partial \Phi}{\partial \lambda}-\frac{1}{a} \frac{\partial \psi}{\partial \phi}, \frac{1}{a} \frac{\partial \Phi}{\partial \phi}+\frac{1}{a \cos \phi} \frac{\partial \psi}{\partial \lambda}\right)
\end{aligned}
$$

onde $\hat{k}$ é o versor na direção vertical. Escrevendo em componentes, segue que:

$$
\begin{aligned}
& u=\frac{i s}{a \cos \phi} \Phi-\frac{1}{a} \frac{\partial \psi}{\partial \phi} \\
& v=\frac{1}{a} \frac{\partial \Phi}{\partial \phi}+\frac{i s}{a \cos \phi} \psi
\end{aligned}
$$

Agora considere as seguintes operações,

$$
\frac{i s}{a \cos \phi}(3.29) \Rightarrow \frac{i s}{a \cos \phi} u=\frac{-s^{2}}{a^{2} \cos \phi^{2}} \Phi-\frac{i s}{a^{2} \cos \phi} \frac{\partial \psi}{\partial \phi}
$$

$\mathrm{e}$

$$
\frac{1}{a \cos \phi} \frac{\partial}{\partial \phi}((3.30) \cos \phi) \Rightarrow \frac{1}{a \cos \phi} \frac{\partial}{\partial \phi}(v \cos \phi)=\frac{1}{a^{2} \cos \phi} \frac{\partial}{\partial \phi}\left(\cos \phi \frac{\partial \Phi}{\partial \phi}\right)+\frac{i s}{a^{2} \cos \phi} \frac{\partial \psi}{\partial \phi}
$$

Somando as duas expressões acima e considerando (3.26), chega-se em:

$$
\frac{1}{a \cos \phi}\left[i s u+\frac{\partial}{\partial \phi}(v \cos \phi)\right]=\vec{\nabla}^{2} \Phi
$$


De forma análoga, fazendo $\left.\left.\frac{1}{a \cos \phi} \frac{\partial}{\partial \phi}(3.29) \cos \phi\right)-\frac{i s}{a \cos \phi} 3.30\right)$, é possível mostrar que:

$$
\frac{1}{a \cos \phi}\left[\frac{-i s}{a \cos \phi} v+\frac{\partial}{\partial \phi}(u \cos \phi)\right]=-\vec{\nabla}^{2} \psi
$$

Tendo em vista a separação de variáveis dada por (3.2), é conveniente fazer a seguinte substituição $u \rightarrow U$ e $v \rightarrow i V$ e, considerando (3.12), as expressões obtidas anteriormente podem ser reescritas como:

$$
\begin{aligned}
U & =\frac{i s}{\cos \phi} \Phi-\frac{1}{a} \frac{\partial \psi}{\partial \phi} \\
i V & =\frac{1}{a} \frac{\partial \Phi}{\partial \phi}+\frac{i s}{a \cos \phi} \psi \\
\vec{\nabla}^{2} \Phi & =\frac{i}{a \cos \phi}\left[s U+\frac{\partial}{\partial \phi}(V \cos \phi)\right]=\frac{i \sigma}{g h_{e}} P \\
\vec{\nabla}^{2} \psi & =\frac{-1}{a \cos \phi}\left[s V+\frac{\partial}{\partial \phi}(U \cos \phi)\right]
\end{aligned}
$$

Agora, com o auxílio das identidades acima, considere as seguintes expressões:

$$
\frac{1}{a \cos \phi}\left[s(3.3)-\frac{\partial}{\partial \phi}(\cos \phi(3.4))\right]=\sigma i \vec{\nabla}^{2} \Phi+2 \Omega \sin \phi \vec{\nabla} \psi-\frac{2 \Omega U}{a} \cos \phi-\vec{\nabla}^{2} P=0
$$

$\mathrm{e}$

$$
\frac{1}{a \cos \phi}\left[-s(3.4)+\frac{\partial}{\partial \phi}(\cos \phi(3.3))\right]=\sigma \vec{\nabla}^{2} \psi+2 \Omega i \sin \phi \vec{\nabla}^{2} \Phi-\frac{2 \Omega}{a} V \cos \phi=0
$$

Substituindo (3.35), 3.36) e 3.37 nas equações acima, chega-se respectivamente em:

$$
\begin{array}{r}
\left(\sigma \vec{\nabla}^{2}-\frac{2 \Omega s}{a^{2}}+\frac{g h_{e}}{\sigma} \vec{\nabla}^{4}\right) i \Phi+\left(2 \Omega \sin \phi \vec{\nabla}^{2}+\frac{2 \Omega \cos \phi}{a^{2}} \frac{\partial}{\partial \phi}\right) \psi=0 \\
\left(\sigma \vec{\nabla}^{2}-\frac{2 \Omega s}{a^{2}}\right) \psi+\left(2 \Omega \sin \phi \vec{\nabla}^{2}+\frac{2 \Omega \cos \phi}{a^{2}} \frac{\partial}{\partial \phi}\right) i \Phi=0
\end{array}
$$

Adimensionando as equações acima, ou seja, tomando $\sigma \rightarrow 2 \Omega \sigma, \vec{\nabla} \rightarrow \vec{\nabla} / a, \Phi \rightarrow$ $2 \Omega /\left(g h_{e}\right)^{1 / 2} \Phi$ e $\psi \rightarrow 2 \Omega /\left(g h_{e}\right)^{1 / 2} \psi$, obtêm-se:

$$
\begin{aligned}
\left(\sigma \vec{\nabla}^{2}-s+\frac{g h_{e}}{\sigma(2 \Omega a)^{2}} \vec{\nabla}^{4}\right) i \Phi+\left(\sin \phi \vec{\nabla}^{2}+\cos \phi \frac{\partial}{\partial \phi}\right) \psi & =0 \\
\left(\sigma \vec{\nabla}^{2}-s\right) \psi+\left(\sin \phi \vec{\nabla}^{2}+\cos \phi \frac{\partial}{\partial \phi}\right) i \Phi & =0
\end{aligned}
$$

Adicionalmente, para simplificar o problema, considera-se a seguinte mudança de variável,

$$
\begin{aligned}
& \sin \phi \equiv \mu \Rightarrow \frac{1}{\cos \phi} \frac{\partial}{\partial \phi}=\frac{\partial}{\partial \mu}, \cos \phi^{2}=1-\mu^{2} \\
& \vec{\nabla}^{2}=\frac{-s^{2}}{1-\mu^{2}}+\frac{\partial}{\partial \mu}\left(\left(1-\mu^{2}\right) \frac{\partial}{\partial \mu}\right)
\end{aligned}
$$


Consequentemente, usando as relações acima, as equações obtidas anteriormente podem ser escritas em função de $\mu$ de acordo com:

$$
\begin{aligned}
\left(\sigma \vec{\nabla}^{2}-s+\frac{g h_{e}}{\sigma(2 \Omega a)^{2}} \vec{\nabla}^{4}\right) i \Phi+\left(\mu \vec{\nabla}^{2}+\left(1-\mu^{2}\right) \frac{\partial}{\partial \mu}\right) \psi & =0 \\
\left(\sigma \vec{\nabla}^{2}-s\right) \psi+\left(\mu \vec{\nabla}^{2}+\left(1-\mu^{2}\right) \frac{\partial}{\partial \mu}\right) i \Phi & =0
\end{aligned}
$$

Definindo o operador $D=\left(1-\mu^{2}\right) \partial / \partial \mu$ e a constante $\gamma=\left(g h_{e}\right)^{1 / 2} /(2 a \Omega)$, o sistema de duas equações que resolvem a estrutura horizontal do problema podem ser reescritas como:

$$
\begin{aligned}
\left(\sigma \vec{\nabla}^{2}-s+\frac{\gamma^{2}}{\sigma} \vec{\nabla}^{4}\right) i \Phi+\left(\mu \vec{\nabla}^{2}+D\right) \psi & =0 \\
\left(\sigma \vec{\nabla}^{2}-s\right) \psi+\left(\mu \vec{\nabla}^{2}+D\right) i \Phi & =0
\end{aligned}
$$

As equações (3.39) e 3.40 acima constituem um sistema de equações análogo ao obtido por Longuet-Higgins (1968). Entretanto, em Kasahara (1976) ressaltou-se que é vantajoso manter a variável $P$ no sistema de equações, ao invés de eliminá-la com o auxílio de (3.37). Então, adimensionando (3.37) e substituindo em (3.39), obtêm-se:

$$
\begin{aligned}
\left(\sigma \vec{\nabla}^{2}-s\right) i \Phi+\left(\mu \vec{\nabla}^{2}+D\right) \psi & =\vec{\nabla}^{2} P \\
\left(\sigma \vec{\nabla}^{2}-s\right) \psi+\left(\mu \vec{\nabla}^{2}+D\right) i \Phi & =0 \\
\sigma P & =-\gamma^{2} \vec{\nabla}^{2}(i \Phi)
\end{aligned}
$$

Em função da condição de regularidade da solução nos polos, imposta pela condição de fronteira (3.24), para resolver o sistema de equações (3.41), 3.42) e (3.43), as variáveis $\Phi$, $\psi$ e $P$ são expandidas em séries de polinômios de Legendre associados $P_{n}^{s}(\mu)$, de grau $n$ e ordem $s$ :

$$
\left(\begin{array}{l}
\Phi \\
\psi \\
P
\end{array}\right)=\sum_{n=s}^{\infty}\left(\begin{array}{c}
i A_{n}^{s} \\
B_{n}^{s} \\
C_{n}^{s}
\end{array}\right) P_{n}^{s}(\mu)
$$

Usando as seguintes propriedades dos polinômios de Legendre associados:

$$
\left\{\begin{array}{l}
\vec{\nabla}^{2} P_{n}^{s}=-n(n+1) P_{n}^{s} \\
\mu P_{n}^{s}=\frac{n+s}{2 n+1} P_{n-1}^{s}+\frac{n-s+1}{2 n+1} P_{n+1}^{s} \\
D P_{n}^{s}=\frac{(n+1)(n+s)}{2 n+1} P_{n-1}^{s}-\frac{n(n-s+1)}{2 n+1} P_{n+1}^{s}
\end{array}\right.
$$


é possível mostrar que

$$
\left(\mu \vec{\nabla}^{2}+D\right) P_{n}^{s}=-\frac{(n-1)(n+1)(n+s)}{2 n+1} P_{n-1}^{s}-\frac{n(n+2)(n-s+1)}{2 n+1} P_{n+1}^{s}
$$

Substituindo 3.44 em 3.41, segue que:

$$
-\left(\sigma \vec{\nabla}^{2}-s\right) \sum_{n=s}^{\infty} A_{n}^{s} P_{n}^{s}+\left(\mu \vec{\nabla}^{2}+D\right) \sum_{n=s}^{\infty} B_{n}^{s} P_{n}^{s}=\vec{\nabla}^{2} \sum_{n=s}^{\infty} C_{n}^{s} P_{n}^{s}
$$

Com o auxílio das propriedades dos polinômios de Legendre associados:

$$
\begin{gathered}
{[\sigma n(n+1)+s] \sum_{n=s}^{\infty} A_{n}^{s} P_{n}^{s}-\frac{(n-1)(n+1)(n+s)}{2 n+1} \sum_{n=s}^{\infty} B_{n}^{s} P_{n-1}^{s}} \\
-\frac{n(n+2)(n-s+1)}{2 n+1} \sum_{n=s}^{\infty} B_{n}^{s} P_{n+1}^{s}=-n(n+1) \sum_{n=s}^{\infty} C_{n}^{s} P_{n}^{s}
\end{gathered}
$$

e fazendo as mudanças de índices $n \rightarrow n+1$ e $n \rightarrow n-1$ no terceiro e quarto termo das séries, respectivamente, chega-se na seguinte relação de recorrência para os coeficientes das séries:

$$
\begin{aligned}
- & \sigma A_{n}^{s}-\frac{s}{n(n+1)} A_{n}^{s}+\frac{(n+2)(n+s+1)}{(2 n+3)(n+1)} B_{n+1}^{s} \\
& +\frac{(n-1)(n-s)}{n(2 n-1)} B_{n-1}^{s}=C_{n}^{s}
\end{aligned}
$$

Seguindo Kasahara (1976), define-se algumas variáveis para simplificar a equação acima:

$$
\begin{aligned}
K_{n} & =\frac{-s}{n(n+1)} \\
p_{n} & =\frac{(n+1)(n+s)}{n(2 n+1)} \\
q_{n} & =\frac{n(n-s+1)}{(n+1)(2 n+1)} \\
r_{n} & =-\gamma^{2} n(n+1)
\end{aligned}
$$

Consequentemente, a relação de recorrência 3.47 torna-se:

$$
-\sigma A_{n}^{s}+K_{n} A_{n}^{s}+p_{n+1} B_{n+1}^{s}+q_{n-1} B_{n-1}^{s}=C_{n}^{s}
$$

De forma totalmente análoga, substituindo (3.44) em 3.42) e 3.43), obtem-se as seguintes relações de recorrência:

$$
\begin{aligned}
-\sigma B_{n}^{s}+K_{n} B_{n}^{s}+p_{n+1} A_{n+1}^{s}+q_{n-1} A_{n-1}^{s} & =0 \\
\sigma C_{n}^{s} & =r_{n} A_{n}^{s}
\end{aligned}
$$


Esse sistema de equações algébricas pode ser separado em dois casos independentes: o primeiro contém os coeficientes $A_{n}^{s}$ e $C_{n}^{s} \operatorname{com} n=s, s+2, s+4, \cdots$ e $B_{n}^{s} \operatorname{com} n=$ $s+1, s+3, s+5, \cdots$. Nesse caso, as perturbações da altura e da velocidade zonal são funções simétricas em relação ao equador e a velocidade meridional é antissimétrica em relação ao equador. Este será chamado de caso simétrico.

O segundo caso, chamado de antissimétrico, contém $A_{n}^{s}$ e $C_{n}^{s}$, com $n=s+1, s+$ $3, s+5, \cdots$ e $B_{n}^{s}$, com $n=s, s+2, s+4, \cdots$. Nesse caso, as perturbações da altura e da velocidade zonal são funções antissimétricas em relação ao equador e a velocidade meridional é simétrica em relação ao equador.

Assim, seja $\mathbf{X}$ um vetor coluna tal que,

$$
\begin{array}{r}
\mathbf{X}=\left(A_{s}^{s}, B_{s+1}^{s}, C_{s}^{s}, A_{s+2}^{s}, B_{s+3}^{s}, C_{s+2}^{s}, \cdots\right. \\
\left.A_{s+2 N}^{s}, B_{s+2 N+1}^{s}, C_{s+2 N}^{s}\right)^{T}
\end{array}
$$

e

$$
\mathbf{A}=\left(\begin{array}{cccccccccc}
K_{s} & p_{s+1} & -1 & 0 & 0 & 0 & 0 & . & . & . \\
q_{s} & K_{s+1} & 0 & p_{s+2} & 0 & 0 & 0 & . & . & . \\
r_{s} & 0 & 0 & 0 & 0 & 0 & 0 & . & . & . \\
0 & q_{s+1} & 0 & K_{s+2} & p_{s+3} & -1 & 0 & . & . & . \\
0 & 0 & 0 & q_{s+2} & K_{s+3} & 0 & p_{s+4} & . & . & . \\
0 & 0 & 0 & r_{s+2} & 0 & 0 & 0 & . & . & . \\
. & . & . & . & . & . & . & . & . & . \\
. & . & . & . & . & . & . & . & . & . \\
. & . & . & . & . & . & . & . & . & .
\end{array}\right)
$$

Logo, o caso simétrico pode ser escrito como:

$$
\mathbf{A X}=\sigma \mathbf{X}
$$

Para o caso antissimétrico, define-se um vetor coluna $\mathbf{Y}$ tal que,

$$
\begin{array}{r}
\mathbf{Y}=\left(B_{s}^{s}, A_{s+1}^{s}, C_{s+1}^{s}, B_{s+2}^{s}, A_{s+3}^{s}, C_{s+3}^{s}, \cdots\right. \\
\left.B_{s+2 N}^{s}, A_{s+2 N+1}^{s}, C_{s+2 N+1}^{s}\right)^{T}
\end{array}
$$


e

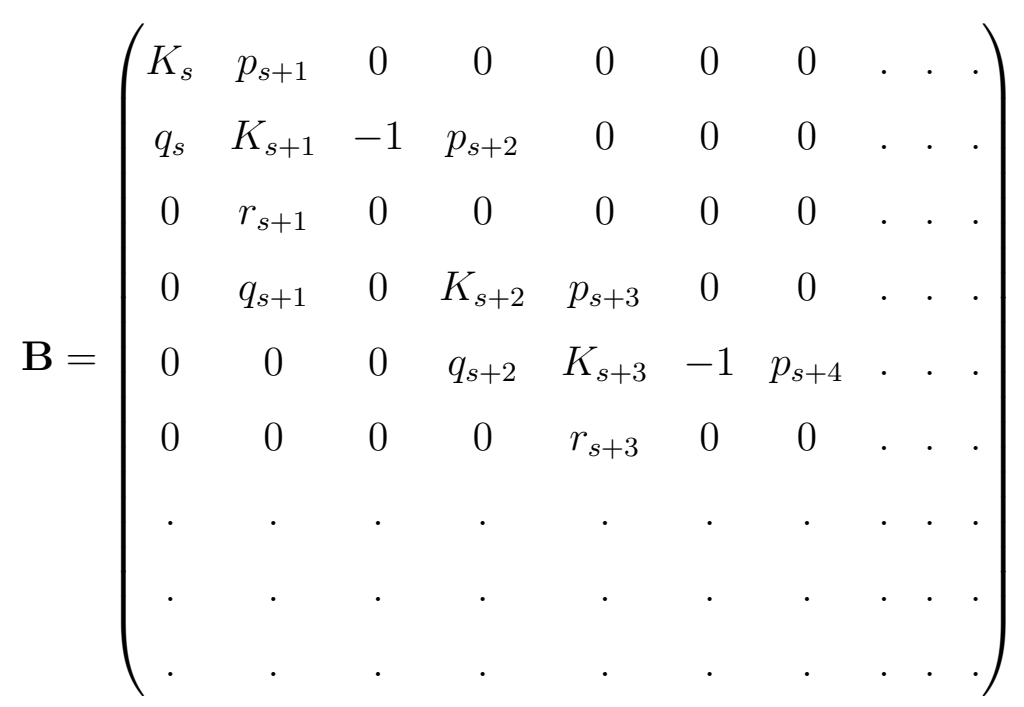

Então, o caso antissimétrico pode ser escrito como:

$$
\mathbf{B Y}=\sigma \mathbf{Y}
$$

O valor da frequência adimensional $\sigma$ é obtido determinando os autovalores das matrizes A ou B. Além disso, com os autovetores X e Y e a expansão em série (3.44) é possível determinar $\Phi, \psi$ e $P$ e, usando 3.39 e (3.40), obtém-se $U$ e $V$. Os autovalores e autovetores das matrizes A e $\mathbf{B}$ são determinados utilizando o método $\mathbf{Q R}$, onde $\mathbf{Q}$ é uma matriz ortogonal e $R$ é uma matriz triangular superior. Neste caso, uma das matrizes A ou B é escrita na forma de Hessenberg superior $\mathbf{H}$ (i.e., uma matriz quase triangular superior, ou seja, os elementos abaixo da diagonal secundária inferior nulos.). Com a matriz $\mathbf{H}=\mathbf{A}_{\mathbf{1}}$, resolve-se o sistema

$$
\begin{aligned}
\mathbf{A}_{\mathbf{i}} & =\mathbf{Q}_{\mathbf{i}} \mathbf{R}_{\mathbf{i}} \\
\mathbf{A}_{\mathbf{i}+\mathbf{1}} & =\mathbf{R}_{\mathbf{i}} \mathbf{Q}_{\mathbf{i}}
\end{aligned}
$$

de forma iterativa. A matriz $\mathbf{A}_{\mathbf{i}}$, após um número finito de iterações, converge para uma matriz triangular superior ou uma matriz em bloco, cujos autovalores podem ser obtidos trivialmente e como a matriz $\mathbf{Q}$ é uma matriz ortogonal, os autovalores da matriz $\mathbf{H}=\mathbf{A}_{\mathbf{1}}$ são preservados durante as iterações (Press et al. 1992). Para os cálculos descritos acima foram utilizadas rotinas Fortran implementadas pelos Drs. José Paulo Bonatti e Pedro Leite da Silva Dias no desenvolvimento de um modelo espectral para as equações da águarasa na esfera descrito em Bonatti e Silva Dias (1983). 


\subsubsection{Determinação das autofrequências e alturas equivalentes, no caso hidrostático}

No caso hidrostático, quando $\delta_{H}=0$, a expressão do autovalor da estrutura vertical (3.17) torna-se:

$$
\lambda=\left(\frac{1}{g h_{e h}}-\frac{1}{C_{s}^{2}}\right) N^{2}
$$

onde $h_{h e h}$ é a altura equivalente para o modelo hidrostático. Como os valores de $\lambda$ são determinados diretamente como autovalores da equação da estrutura vertical a partir de (3.19), então $h_{e h}$ é obtida diretamente pela equação acima:

$$
\begin{aligned}
h_{e h} & =\frac{C_{s}^{2}}{g}\left(1+\frac{C_{s}^{2} \lambda_{k}}{N^{2}}\right)^{-1} \\
& =\frac{4 \kappa H}{1+4 \hat{k}^{2} H^{2}}
\end{aligned}
$$

Com a altura equivalente determinada, para obter as soluções características da versão hidrostática do modelo, basta resolver o problema de autovalor da estrutura horizontal (3.12), com condições de contorno (3.24). As propriedades dessas soluções características são discutidas em profundidade em Kasahara (1976); Bonatti et al. (1983).

\subsubsection{Determinação das autofrequências e alturas equivalentes, no caso não-hidrostático}

No caso não hidrostático, quando $\delta_{H}=1$, os problemas de autovalor das estruturas vertical e horizontal constituem um sistema de equações acopladas para $\sigma$ e $h_{e}$. Kasahara e Qian (2000) propuseram um método iterativo para resolver esse problema de autovalor . O parâmetro $\delta_{H}$ será mantido para possibilitar o estudo das alterações dos modos normais devido a ausência da aproximação hidrostática. Como $\delta_{H}$ é diferente de zero, a expressão (3.17) pode ser reescrita como:

$$
\delta_{H} \sigma^{2}=N^{2}-\frac{C_{s}^{2} g h_{e} \lambda_{k}}{\left(C_{s}^{2}-g h_{e}\right)}
$$

Além disso, ao resolver o problema da estrutura horizontal, para cada altura equivalente $h_{e}$, número de onda zonal $s$ e índice meridional $l$, que distingue a estrutura meridional dos modos, determina-se uma autofrequência como autovalor da equação da maré de Laplace dada por (3.12). Assim, a frequência obtida do problema de autovalor do operador da Maré de Laplace, com as condições de contorno (3.24), será representada como: 


$$
\sigma=F\left(h_{e}, s, l\right)
$$

Ou seja, para o caso não-hidrostático, a frequência e a altura equivalente devem ser determinadas resolvendo as equações (3.59) e (3.60) de forma simultânea. Embora a altura equivalente não seja um parâmetro constante no regime não-hidrostático, ela é útil para identificar os diferentes modos normais. Se $C_{s}^{2}>g h_{e}$, por (3.59), tem-se:

$$
\delta_{H} \sigma^{2}<N^{2}
$$

ou seja, a frequência do modo é menor que a frequência de Brunt-Väisälä. Este regime representa os modos de gravidade-inerciais.

Por outro lado, se $C_{s}^{2}<g h_{e}$, por (3.59), tem-se:

$$
\delta_{H} \sigma^{2}>N^{2}
$$

Nesse caso, a frequência do modo é maior que a frequência de Brunt-Väisälä, e tal condição representa o regime de oscilação dos modos acústico-inerciais.

Note que a relação entre $h_{e}$ e $\sigma$ é transcendental, ou seja, não há uma expressão analítica para $F$ em 3.60 . Por isso não é possível resolver o sistema eliminando diretamente o parâmetro $h_{e}$ das equações (3.60) e (3.59). Desse fato, surge a necessidade de um método iterativo que resolva as equações 3.60 e 3.59 simultaneamente. Kasahara e Qian (2000) propuseram um método iterativo que utiliza chutes iniciais convenientes de modo a obter a convergência para a solução do sistema num tempo de máquina razoável. Esses chutes inicias são determinados a partir de expressões analíticas aproximadas para a autofrequência associada as funções de Hough (3.60).

Neste contexto, Longuet-Higgins (1968) mostrou que para valores grandes de $h_{e}$, tem-se a seguinte aproximação assintótica de (3.60):

$$
\sigma^{2}+\frac{2 \Omega s}{n(n+1)} \sigma-\frac{n(n+1)}{a^{2}} g h_{e}=0
$$

onde $s$ é o número de onda zonal e $n$ é a ordem do polinômios de Legendre associados, sendo $n \geq s$. Nessa expressão $n$ é um índice meridional, tal que o primeiro modo meridional 
é $n=s$. O segundo modo corresponde ao índice $n=s+1$ e assim por diante. Resolvendo a equação quadrática (3.63) para $\sigma$, obtém-se valores aproximados para as frequências das oscilações de primeira espécie, ou seja, para as ondas gravito-inerciais.

Ainda na mesma ordem de aproximação, Longuet-Higgins (1968) obteve a seguinte expressão para a frequência das oscilações de segunda espécie:

$$
\sigma=-\frac{2 \Omega s}{n(n+1)}
$$

Além disso, a equação 3.59 pode ser reescrita como:

$$
h_{e}=\frac{C_{s}^{2}}{g}\left(1+\frac{C_{s}^{2} \lambda_{k}}{N^{2}-\delta_{H} \sigma^{2}}\right)
$$

Como a frequência das oscilações de segunda espécie tem valores muito menores que a frequência de Brunt-Väisälä, então os valores da altura equivalente para esses modos pode ser aproximada por (3.58). O mesmo não pode ser feito para as oscilações de primeira (gravito-inerciais) e terceira espécie (acústico-inerciais), pois em ambos os casos a frequência dos modos tem valores da ordem de grandeza da frequência de Brunt-Väisälä. Devido a esse fato, substituindo (3.65) em (3.63) obtém-se:

$$
\left(\sigma^{2}+A \sigma\right)\left(N^{2}-\delta_{H} \sigma^{2}+C_{s}^{2} \lambda_{k}\right)-B\left(N^{2}-\delta_{H} \sigma^{2}\right)=0
$$

ou, alternativamente,

$$
\delta_{H} \sigma^{4}+\delta_{H} A \sigma^{3}-\left(\lambda_{k} C_{s}^{2}+N^{2}+\delta_{H} B\right) \sigma^{2}-A\left(N^{2}+C_{s}^{2} \lambda_{k}\right) \sigma+B N^{2}=0
$$

onde

$$
A=\frac{2 \Omega s}{n(n+1)} \text { e } B=\frac{n(n+1)}{a^{2}} C_{s}^{2}
$$

A equação (3.66) possui dois pares de raízes reais. Um par corresponde às oscilações de leste e de oeste com frequência muito alta, que satisfaz o regime 3.62 e consequentemente representa o modo acústico-inercial. O outro par corresponde às oscilações de leste e de oeste com baixa frequência, satisfazendo (3.61), ou seja, representa o modo gravidadeinercial. Note que, uma vez obtida a frequência pela equação (3.66), basta substituir o valor dessa frequência em (3.65) para obter a altura equivalente do modo correspondente. 
No método iterativo proposto por Kasahara e Qian (2000), a altura equivalente $h_{e}$ é utilizada como parâmetro de iteração. Ou seja, a partir de um chute inicial de $h_{e}$, representado por $h_{e}^{0}$, altera-se o valor de $h_{e}$ até que os valores de $\sigma$, calculados em 3.60 e (3.59) sejam próximos o suficiente (i.e. a diferença entre os valores seja menor que uma tolerância especificada). Longuet-Higgins (1968) mostrou numericamente que a frequência de Hough (i.e., calculada por (3.60) ) é uma função crescente em relação a altura equivalente. Além disso, derivando (3.59) em relação a $h_{e}$, obtemos:

$$
\frac{\partial\left(\delta_{H} \sigma^{2}\right)}{\partial h_{e}}=-\frac{g C_{s}^{4} \lambda_{k}}{\left(C_{s}^{2}-g h_{e}\right)}<0
$$

Como os autovalores $\lambda_{k}$ da estrutura vertical são sempre positivos, a derivada acima demonstra que a frequência em (3.59) é uma função decrescente em relação a $h_{e}$. Logo a intersecção das curvas $\sigma\left(h_{e}, k, s, l\right)$ dadas por (3.59) e (3.60) se cruzam e, consequentemente, a solução deste sistema sempre existe.

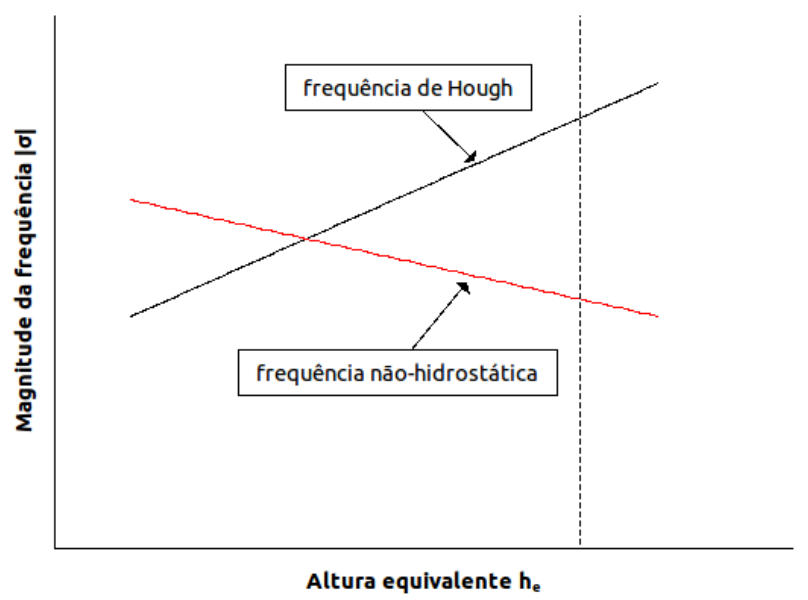

Figura 3.1: Diagrama esquematizando o método iterativo descrito no texto. A linha tracejada indica a altura equivalente inicial. A curva representando a frequência de Hough é a obtida em (3.60). A frequência não-hidrostática é obtida em 3.59 .

A Figura 3.1 mostra esquematicamente o método iterativo utilizado. A linha tracejada indica o valor inicial de $h_{e}$. A partir desse $h_{e}$ inicial, deve-se adicionar ou subtrair um valor $\Delta h_{e}$ até encontrar a altura equivalente tal que as frequências 3.60 e 3.59) sejam iguais, dentro de uma tolerância especificada, ou seja, até encontrar a intersecção das duas curvas da Figura 3.1. Em suma, os valores iniciais devem estar próximos à intersecção das curvas 
e deve-se saber como variar o valor de $h_{e}$ em cada iteração para que a solução seja obtida num tempo de máquina razoável.

O dois métodos iterativos, A e B, propostos por Kasahara e Qian (2000) serão descritos. Os métodos diferem basicamente pelo chute inicial escolhido. No método A, inicia-se com uma altura equivalente inicial $h_{e}^{0}$ obtida a partir de (3.65) com $\sigma$ dado por uma das raízes de (3.67). Agora calcula-se as frequências com 3.60) e (3.59), que serão denotadas por $\sigma_{H}$ e $\sigma_{n}$, respectivamente:

$$
\sigma_{H}^{0}=\sigma_{H}\left(h_{e}^{0}, s, l\right) \text { e } \sigma_{n}^{0}=\sigma_{n}\left(h_{e}^{0}, s, l\right)
$$

O próximo passo é identificar se $h_{e}^{0}$ está à esquerda ou à direta da intersecção. Para isso é feita a seguinte checagem:

$$
\begin{gathered}
\left|\sigma_{H}^{0}\right|>\left|\sigma_{n}^{0}\right| \rightarrow h_{e}^{0} \text { está à esquerda da intersecção }\left(\Delta h_{e}>0\right) \\
\left|\sigma_{H}^{0}\right|<\left|\sigma_{n}^{0}\right| \rightarrow h_{e}^{0} \text { está à direita da intersecção }\left(\Delta h_{e}<0\right)
\end{gathered}
$$

Agora o próximo valor da altura equivalente será $h_{e}^{1}=h_{e}^{0}+\Delta h_{e}$. Esse processo será repetido até o momento em que o ponto de intersecção das curvas for ultrapassado:

$$
\begin{aligned}
& \left|\sigma_{H}^{i}\right|>\left|\sigma_{n}^{i}\right| \rightarrow\left|\sigma_{H}^{i+1}\right|<\left|\sigma_{n}^{i+1}\right| \\
& \left|\sigma_{H}^{i}\right|<\left|\sigma_{n}^{i}\right| \rightarrow\left|\sigma_{H}^{i+1}\right|>\left|\sigma_{n}^{i+1}\right|
\end{aligned}
$$

Quando ocorrer essa troca de sinal, tomar-se-a $h_{e}=\left(h_{e}^{i}+h_{e}^{i+1}\right) / 2$ e por fim, a frequência do modo poderá ser determinada. Note que o índice $i$ indica a i-ésima iteração.

No método B, escolhe-se um modo caracterizado por $(s, l, k)$, onde $s$ denota o número de onda zonal, $l$ o índice meridional que distingue a estrutura meridional dos modos normais, e $k$ indica o modo vertical. Em seguida toma-se um valor inicial para a altura equivalente $h_{e}^{0}$, através de 3.58 ou 3.65 com $\sigma$ dada por uma das menores raízes de (3.66). A partir desse valor, calcula-se a frequência do modo via (3.60), obtendo $\sigma^{0}$. Com esse valor de $\sigma$, calculamos outra altura equivalente $h_{e}^{1}$, utilizando a equação (3.65). Com esse novo valor para a altura equivalente, repete-se o procedimento até que a solução convirja. Esquematicamente o método B é representado da seguinte forma: 


$$
\sigma^{0}=\Im\left(h_{e}^{0}, s, l\right) \rightarrow h_{e}^{1}=L\left(\sigma^{0}, s, l\right) \rightarrow \sigma^{1}=\Im\left(h_{e}^{1}, s, l\right) \rightarrow \ldots
$$

onde $L=L(\sigma, s, l)$ é a altura equivalente, calculada com a equação 3.65) e $\Im$ é a frequência calculada com (3.60). O critério de parada é $\left|h_{e}^{i+1}-h_{e}^{i}\right| \leq \epsilon$, sendo $\epsilon$ a tolerância especificada.

Para os modos acústicos-inerciais, resolve-se (3.66) e uma das duas raízes no regime (3.62) é escolhida, depois calcula-se $h_{e}^{0}$ substituindo essa frequência em (3.65). Com esse valor inicial da altura equivalente, o método A é aplicado.

A determinação da altura equivalente inicial $h_{e}^{0}$ para os modos gravito-inerciais é feita de forma análoga ao procedimento descrito acima para os modos acústico-inerciais. Entretanto, ao resolver a equação (3.66), toma-se uma das raízes no regime (3.61). Para os modos gravito-inerciais com número de onda $s \geq 400$, o método A é utilizado. Para os modos gravito-inerciais com número de onda $s<50$, utiliza-se o método B com $h_{e}^{0}$ obtido de 3.58. E para os modos com número de onda $50<s<400$, utiliza-se o método B, mas com $h_{e}^{0}$ dada por 3.65 com $\sigma$ dada por uma das menores raízes de 3.66.

Para os modos rotacionais(oscilações de segunda espécie), é possível aplicar o método B. Entretanto as soluções na aproximação hidrostática são precisas o suficiente, de acordo com Kasahara e Qian (2000).

\subsubsection{Ortogonalidade e energia dos modos}

Neste item da presente seção será apresentada a demonstração da ortogonalidade dos modos normais do sistema (3.1) bem como a dedução da expressão para a energia dos mesmos realizadas por Kasahara e Qian (2000). Assim, seguindo Kasahara e Qian (2000), considere que as variáveis prognósticas $u^{\prime}, v^{\prime}, w^{\prime}, p^{\prime}$ e $\theta^{\prime}$ são proporcionais a $e^{\nu t}$, tal que $\partial / \partial t \rightarrow \nu$. Assim, as equações governantes linearizadas para o j-ésimo modo podem ser 
escritas como(omitindo o símbolo '),

$$
\begin{aligned}
\nu_{j} \rho_{0} u_{j} & =-\frac{1}{a \cos \phi} \frac{\partial p_{j}}{\partial \lambda}+f \rho_{0} v_{j} \\
\nu_{j} \rho_{0} v_{j} & =-\frac{1}{a} \frac{\partial p_{j}}{\partial \lambda}-f \rho_{0} u_{j} \\
\delta_{H} \nu_{j} \rho_{0} w_{j} & =\theta_{j}-\frac{\partial p_{j}}{\partial z}-\frac{g}{C_{s}^{2}} p_{j} \\
\frac{\nu_{j}}{\rho_{0} N^{2}} \theta_{j} & =-w_{j} \\
\frac{\nu_{j}}{\rho_{0} C_{s}^{2}} p_{j} & =-\nabla \cdot \vec{V}_{j}-\frac{\partial w_{j}}{\partial z}+\frac{g}{C_{s}^{2}} w_{j}
\end{aligned}
$$

Considere as mesmas equações (3.70)-3.74 para as variáveis $u_{k}^{*}, v_{k}^{*}, w_{k}^{*}, \theta_{k}^{*}$ e $p_{k}^{*}$ e, onde o símbolo $*$ representa o complexo conjugado de sua respectiva variável,

$$
\begin{aligned}
\nu_{j} \rho_{0} u_{k}^{*} & =-\frac{1}{a \cos \phi} \frac{\partial p_{k}^{*}}{\partial \lambda}+f \rho_{0} v_{k}^{*} \\
\nu_{j} \rho_{0} v_{k}^{*} & =-\frac{1}{a} \frac{\partial p_{k}^{*}}{\partial \lambda}-f \rho_{0} u_{k}^{*} \\
\delta_{H} \nu_{j} \rho_{0} w_{k}^{*} & =\theta_{k}^{*}-\frac{\partial p_{k}^{*}}{\partial z}-\frac{g}{C_{s}^{2}} p_{k}^{*} \\
\frac{\nu_{j}}{\rho_{0} N^{2}} \theta_{k}^{*} & =-w_{k}^{*} \\
\frac{\nu_{j}}{\rho_{0} C_{s}^{2}} p_{k}^{*} & =-\nabla \cdot \vec{V}_{k}^{*}-\frac{\partial w_{k}^{*}}{\partial z}+\frac{g}{C_{s}^{2}} w_{k}^{*}
\end{aligned}
$$

Multiplicando as equações 3.70-3.74 por $u_{k}^{*}, v_{k}^{*}, w_{k}^{*}, \theta_{k}^{*}$ e $p_{k}^{*}$, respectivamente, e analogamente multiplicando as equações 3.75-3.79 por $u_{j}, v_{j}, w_{j}, \theta_{j}$ e $p_{j}$ respectivamente. Somando esses dois conjuntos de equações obtém-se a seguinte relação,

$$
\begin{aligned}
& \left(\nu_{j}+\nu_{k}^{*}\right)\left[\rho_{0}\left(u_{j} u_{k}^{*}+v_{j} v_{k}^{*}+\delta_{H} w_{j} w_{k}^{*}\right)+\frac{1}{\rho_{0} N^{2}} \theta_{j} \theta_{k}^{*}+\frac{1}{\rho_{0} C_{s}^{2}} p_{j} p_{k}^{*}\right]= \\
= & -\nabla \cdot\left(p_{j} \vec{V}_{k}^{*}+p_{k}^{*} \vec{V}_{j}\right)-\frac{\partial}{\partial z}\left(w_{j} p_{k}^{*}+w_{k}^{*} p_{j}\right)
\end{aligned}
$$

O próximo passo é integrar a última expressão no volume $V$ que está sobre uma esfera de raio $a$, ou seja, $V=(\lambda, \phi, z) \in \mathbb{R}^{3}: \lambda \in[0,2 \pi], \phi \in[-\pi / 2, \pi / 2], z \in\left[a, a+z_{T}\right]$. Além disso, a velocidade vertical $w$ deve ser nula tanto na superfície quanto no topo (condições de contorno). O segundo termo do lado direito da equação é trivialmente anulado, devido as condições de contorno. O primeiro termo do lado direito da equação é anulado com a utilização do teorema da divergência de Gauss e as condições de contorno horizontais. Assim a expressão resultante é: 


$$
\left(\nu_{j}+\nu_{k}^{*}\right) \int_{V}\left[\frac{\rho_{0}}{2}\left(u_{j} u_{k}^{*}+v_{j} v_{k}^{*}+\delta_{H} w_{j} w_{k}^{*}\right)+\frac{\theta_{j} \theta_{k}^{*}}{2 \rho_{0} N^{2}}+\frac{p_{j} p_{k}^{*}}{2 \rho_{0} C_{s}^{2}}\right] d V=0
$$

onde $d V=a^{2} \cos \phi d \phi d \lambda d z$.

Para uma solução não trivial a integral da energia total, ET, para o j-ésimo modo deve ser positiva. Isso ocorre quando $k=j$. Então:

$$
E T_{j}=K_{j}+E_{j}+A_{j}>0
$$

onde

$$
\begin{aligned}
K_{j} & =\int_{V} \frac{\rho_{0}}{2}\left(u_{j} u_{j}^{*}+v_{j} v_{j}^{*}+\delta_{H} w_{j} w_{j}^{*}\right) d V=\int_{V} \frac{\rho_{0}}{2}\left(\left|u_{j}\right|^{2}+\left|v_{j}\right|^{2}+\delta_{H}\left|w_{j}\right|^{2}\right) d V \\
E_{j} & =\int_{V} \frac{p_{j} p_{j}^{*}}{2 \rho_{0} C_{s}^{2}} d V=\int_{V} \frac{\left|p_{j}\right|^{2}}{2 \rho_{0} C_{s}^{2}} d V \\
A_{j} & =\int_{V} \frac{\theta_{j} \theta_{j}^{*}}{2 \rho_{0} N^{2}} d V=\int_{V} \frac{\left|\theta_{j}\right|^{2}}{2 \rho_{0} N^{2}} d V
\end{aligned}
$$

Dado que $E T_{j} \neq 0$, por (3.81), tem-se que $\nu_{j}+\nu_{j}^{*}=0$, portanto a parte real de $\nu_{j}$ deve ser nula. Logo, $\nu_{j}$ é imaginário puro, consequentemente para $j \neq k$ tem-se $\nu_{j}+\nu_{k}^{*} \neq 0$. Disso obtém-se a seguinte relação de ortogonalidade:

$$
\int_{V}\left[\frac{\rho_{0}}{2}\left(u_{j} u_{k}^{*}+v_{j} v_{k}^{*}+\delta_{H} w_{j} w_{k}^{*}\right)+\frac{\theta_{j} \theta_{k}^{*}}{2 \rho_{0} N^{2}}+\frac{p_{j} p_{k}^{*}}{2 \rho_{0} C_{s}^{2}}\right] d V=0
$$

para $j \neq k$.

As integrais $K_{j}, A_{j}$ e $E_{j}$ representam as energias cinética, termobárica e elástica, respectivamente.

\subsubsection{Cálculo das Energias}

A energia cinética pode ser decomposta em três componentes: energia cinética zonal $E K U_{j}$, meridional $E K V_{j}$ e vertical $E K W_{j}$, onde

$$
\begin{aligned}
E K U_{j} & =\int_{V} \frac{\rho_{0}}{2}\left|u_{j}\right|^{2} d V \\
E K V_{j} & =\int_{V} \frac{\rho_{0}}{2}\left|v_{j}\right|^{2} d V \\
E K W_{j} & =\int_{V} \frac{\rho_{0}}{2} \delta_{H}\left|w_{j}\right|^{2} d V
\end{aligned}
$$


Substituindo o ansatz de onda plana (3.2) na expressão para a energia total (3.82), obtém-se:

$$
\begin{aligned}
E T_{j} & =\int_{0}^{2 \pi} \int_{-\pi / 2}^{\pi / 2} \int_{a}^{a+z_{T}} a^{2} \cos \phi d \phi d \lambda d z\left\{\frac{1}{2}\left(\left|U_{j}\right|^{2}+\left|V_{j}\right|^{2}\right) \xi_{j} \xi_{j}^{*}+\frac{\delta_{H}}{2}\left|P_{j}\right|^{2} \eta_{j} \eta_{j}^{*}+\frac{1}{2} \frac{\left|P_{j}\right|^{2}}{C_{s}^{2}} \xi_{j} \xi_{j}^{*}\right. \\
& \left.+\frac{1}{2 N^{2}}\left|P_{j}\right|^{2} \theta_{j} \theta_{j}^{*}\right\}
\end{aligned}
$$

Rearranjando os termos da expressão acima:

$$
\begin{aligned}
E T_{j} & =\pi a^{2}\left[\int_{-\pi / 2}^{\pi / 2}\left(\left|U_{j}\right|^{2}+\left|V_{j}\right|^{2}+\frac{\left|P_{j}\right|^{2}}{C_{s}^{2}}\right) \cos \phi d \phi\right] \int_{a}^{a+z_{T}} \xi_{j} \xi_{j}^{*} d z \\
& +\pi a^{2} \delta_{H}\left[\int_{-\pi / 2}^{\pi / 2}\left|P_{j}\right|^{2} \cos \phi d \phi\right] \int_{a}^{a+z_{T}} \eta_{j} \eta_{j}^{*} d z \\
& +\pi a^{2} \frac{g^{2}}{N^{2}}\left[\int_{-\pi / 2}^{\pi / 2}\left|P_{j}\right|^{2} \cos \phi d \phi\right] \int_{a}^{a+z_{T}} \theta_{j} \theta_{j}^{*} d z
\end{aligned}
$$

A integrais que estão entre colchetes foram calculadas com o método da quadratura de Gauss. A integrais na vertical foram calculadas analiticamente. Substituindo as autofunções da estrutura vertical para o caso isotérmico e utilizando a seguinte substituição $\theta_{j}(z):=\xi_{j}(z) g / C_{s}^{2}-\zeta_{j}(z) g$, as integrais na vertical resultam em:

$$
\begin{aligned}
\int_{a}^{a+z_{T}} \xi_{j} \xi_{j}^{*} d z & =\left(\frac{1}{C_{s}^{2}}-\frac{1}{g h_{e}}\right)^{-2} \frac{A_{j}^{2}}{\sigma^{2}} \frac{1}{2} z_{T} \lambda_{j} \\
\int_{a}^{a+z_{T}} \xi_{j} \eta_{j}^{*} d z & =\left(\frac{1}{C_{s}^{2}}-\frac{1}{g h_{e}}\right)^{-1} \frac{A_{j}^{2}}{\sigma}\left(-\frac{\Gamma}{2} z_{T}\right) \\
\int_{a}^{a+z_{T}} \eta_{j} \eta_{j}^{*} d z & =A_{j}^{2} \frac{1}{2} z_{T} \\
\int_{a}^{a+z_{T}} \xi_{j} \zeta_{j}^{*} d z & =\frac{1}{C_{s}^{2}} \int_{a}^{a+z_{T}} \xi_{j} \xi_{j}^{*} d z-\frac{N^{2}}{g \sigma} \int_{a}^{a+z_{T}} \xi_{j} \eta_{j}^{*} d z \\
\int_{a}^{a+z_{T}} \zeta_{j}^{*} \zeta_{j} d z & =\int_{a}^{a+z_{T}}\left(\frac{1}{C_{s}^{2}} \xi_{j}^{*} \xi_{j}-\frac{1}{C_{s}^{2}} \frac{N^{2}}{g \sigma} \xi_{j}^{*} \eta_{j}+\frac{N^{4}}{g^{2} \sigma^{2}} \eta_{j}^{*} \eta_{j}\right) d z
\end{aligned}
$$

\subsection{Energética dos Modos Acústico-Inerciais e Gravito-Inerciais}

Kasahara e Qian (2000) mostraram que os modos rotacionais não hidrostáticos são praticamente idênticos aos modos do caso hidrostático. Podemos chegar a essa mesma conclusão vendo que o primeiro termo da equação (3.5), responsável pelo efeito nãohidrostático, é muito pequeno para os modos rotacionais. Portanto, iremos analisar apenas a energética dos modos acústico-inerciais e gravito-inerciais. Neste estudo foram considerados os modos com propagação para leste com número de onda zonal variando entre $s=1$ 
até $s=700$, índice meridional $l=0$ e $l=2$ (primeiro e segundo modos simétricos) e para o modo vertical $k=1$.

\subsubsection{Relação de Dispersão e Diagramas da Altura Equivalente}

A relação entre a frequência e o número de onda é conhecida como relação de dispersão. A partir da relação de dispersão de um tipo de onda é possível obter várias informações sobre a mesma. Por exemplo, se o determinante do Hessiano da relação de dispersão for diferente de zero, a menos de um conjunto de medida nula, então a onda representada por essa relação de dispersão é dita dispersiva (Whitham, 1974; Majda, 2003). Além disso, com a relação de dispersão é possível determinar os possíveis tripletos ressonantes resultantes das interações fracamente não-lineares entre ondas que satisfazem algumas condições de ressonância Raupp et al. (2008).

Analisando a relação de dispersão e o diagrama da altura equivalente nas Figuras 3.2 e 3.3. respectivamente, é possível ver com clareza a diferença entre os dois regimes 3.62 e (3.61) que representam os modos acústico-inerciais e gravito-inerciais, respectivamente. Não há diferença significativa entre a relação de dispersão os modos com índice meridional $l=0$ e $l=2$. A altura equivalente das oscilações de terceira espécie (ondas acústicoinerciais) decai rapidamente em relação ao número de onda zonal, enquanto para as oscilações de primeira espécie (ondas gravito-inerciais) a altura equivalente é praticamente constante em relação ao número de onda zonal.

\subsubsection{Energética dos Modos Não-Hidrostáticos}

A energia dos modos é calculada com a expressão (3.91). As energias dos modos acústico-inerciais e gravito-inerciais com índice meridional $l=0$ e modo vertical $k=1$ estão nas Figuras 3.4 e 3.5, respectivamente.

Os modos gravito-inerciais com propagação para leste e com o primeiro modo meridional $l=0$ representam os modos de Kelvin (Matsuno, 1966; Kasahara, 1976). Para os modos gravito-inerciais (oscilações de primeira espécie) é possível ver que o valor da energia total de cada modo permanece praticamente constante em relação ao número de onda zonal, assim como a altura equivalente desses modos. Por outro lado, para os modos acústicoinerciais, da mesma forma que a altura equivalente desses modos diminui com o aumento do número de onda zonal, a energia total diminui em relação ao número de onda zonal. 


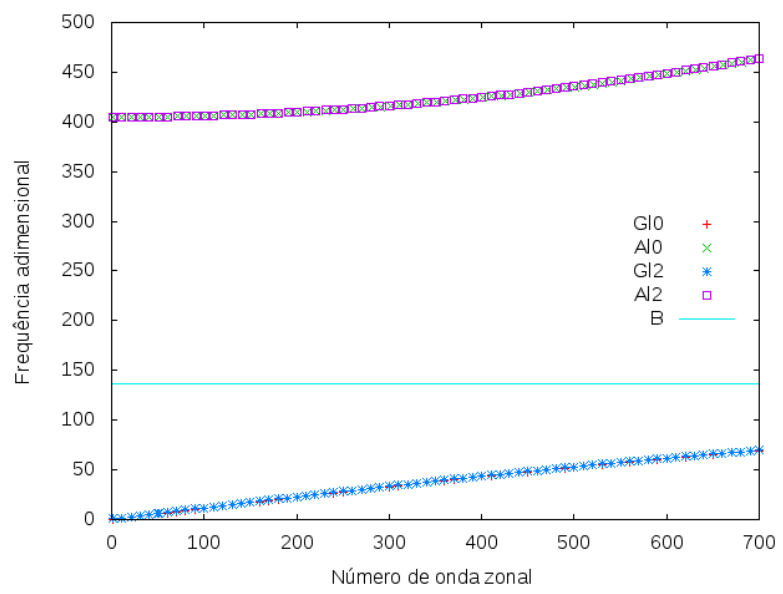

Figura 3.2: Relação de dispersão para os modos gravito-inerciais e acústico-inerciais para o modelo nãohidrostático com modo vertical $k=1$. Os símbolos Gl0 e Gl2 representam os modos gravito-inerciais com índice meridional $l=0$ e $l=2$, respectivamente. Os símbolos Al0 e Al2 representam os modos acústico-inerciais com índice meridional $l=0$ e $l=2$, respectivamente. $B$ representa a frequência de Brunt-Väisälä adimensional $(N / 2 \Omega)$.

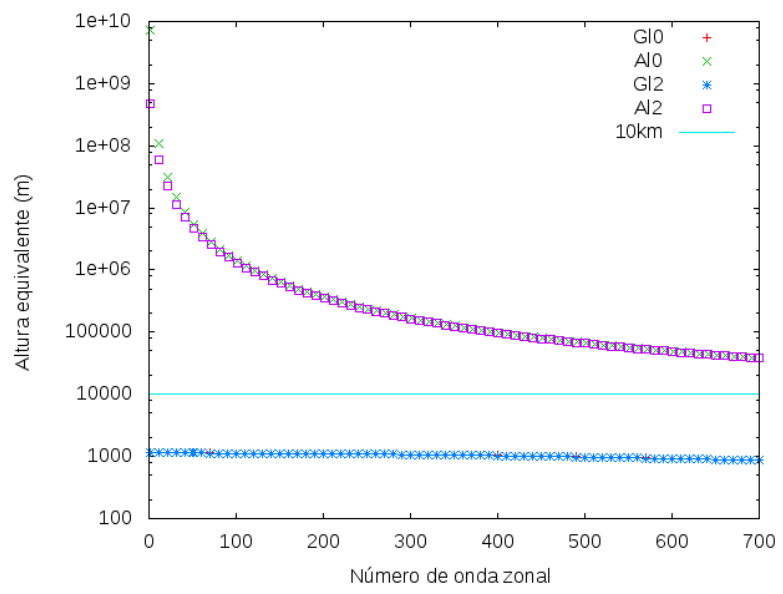

Figura 3.3: Diagrama da altura equivalente para os modos gravito-inerciais e acústico-inerciais com modo vertical $k=1$. Os símbolos Gl0 e Gl2 representam os modos gravito-inerciais com índice meridional $l=0$ e $l=2$, respectivamente. Os símbolos Al0 e Al2 representam os modos acústico-inerciais com índice meridional $l=0$ e $l=2$, repectivamente. E a linha contínua em $10 \mathrm{~km}$ representa a altura equivalente do modo externo, que separa os regimes de gravidade e acústico.

No intuito de analisar a importância de cada tipo de energia em relação a energia total, calculou-se o valor de cada tipo de energia divido pela energia total. Os resultados são ilustrados nas Figuras 3.6 e 3.10.

Da Figura 3.6, nota-se que as energias cinética vertical e elástica são as mais importantes para as oscilações de terceira espécie (acústico inercial). Para números de onda zonal menores que 400, a energia termobarica é mais importante do que as energias cinética 


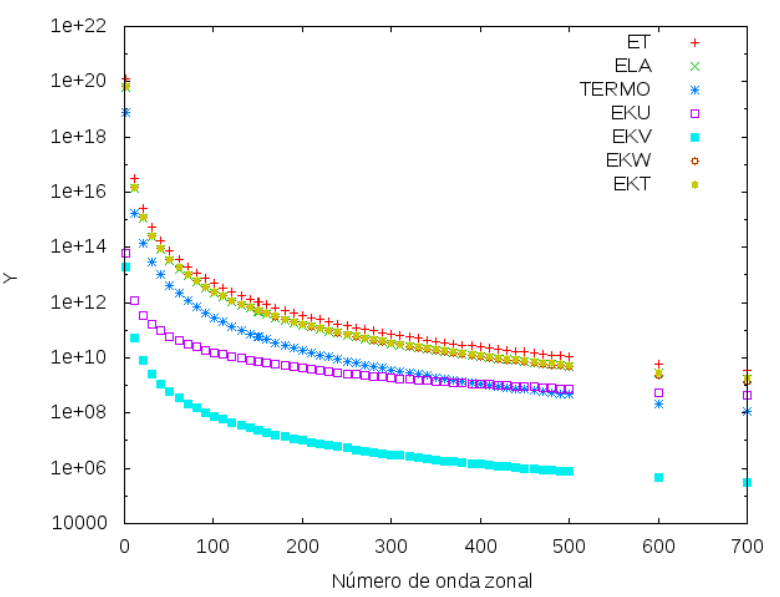

Figura 3.4: Energética dos modos acústico-inerciais com índice meridional $l=0$ e modo vertical $k=$ 1, onde ET, ELA, TERMO, EKU, EKV, EKW e EKT correspondem as energias total, elástica, termobarica, cinética zonal, cinética meridional, cinética vertical e cinética total, respectivamente. As energias são dadas em $J / a^{2} \pi$.

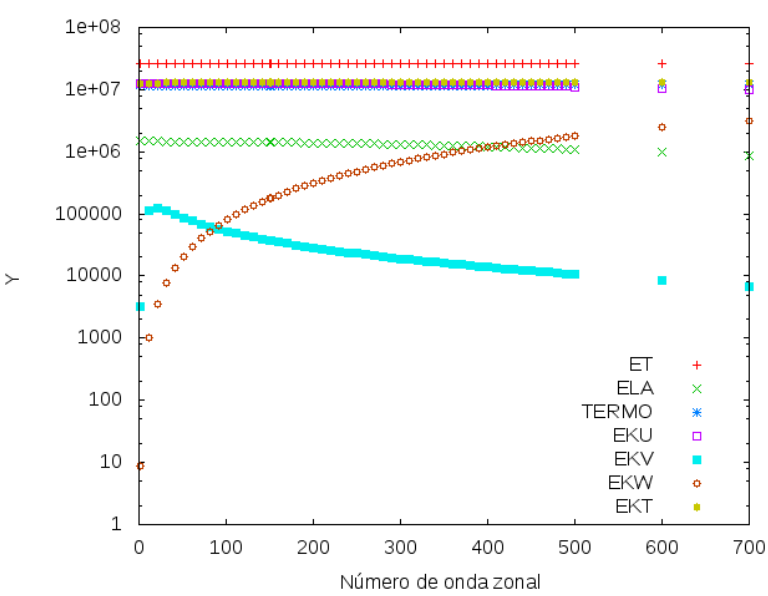

Figura 3.5: Energética dos modos gravito-inerciais com índice meridional $l=0$ e modo vertical $k=1$, onde $E T, E L A, T E R M O, E K U, E K V, E K W$ e EKT correspondem as energias total, elástica, termobarica, cinética zonal, cinética meridional, cinética vertical e cinética total, respectivamente. As energias são dadas em $J / a^{2} \pi$.

zonal e meridional. A energia cinética meridional é muito menor que todos outros tipos de energia. A energia cinética zonal é da mesma ordem de grandeza que a energia cinética meridional para os primeiros números de onda zonal. Entretanto, para números de onda maiores que 400, a energia cinética zonal torna-se mais importante que a energia termobarica. Para analisar o comportamento dos energias cinética vertical e elástica, foi feito uma ampliação na parte superior da Figura 3.6. Pela Figura 3.8 é possível ver que a energia cinética total mantém-se praticamente constante e devido ao aumento da relevância da 
energia cinética zonal e meridional com o aumento do número de onda zonal, a energia cinética vertical tende a diminuir com o aumento de $s$, enquanto a energia elástica tende a aumentar com o aumento de $s$.

Para os modos gravito-inerciais, da Figura 3.7, tem-se que a componente zonal da energia cinética e a energia termobarica correspondem à maior parcela da energia total para todos os números de onda zonal. Dentre as energias elástica, cinética vertical e meridional, a energia elástica é a mais importante para modos com número de onda $s<400$, enquanto para modos com número de onda $s>400$ a energia cinética vertical passa a ter mais importância do que a energia elástica. A componente meridional da energia cinética é muito pequena. Entretanto, ela apresenta um comportamento crescente até o número de onda $s=20$ e após esse valor de $s$, a energia cinética meridional decresce com o aumento de $s$. É interessante notar que inicialmente a energia cinética vertical é menos importante do que a energia cinética meridional, mas para números de onda $s>90$, a componente vertical torna-se mais importante que a componente meridional. Ampliando a parte superior da figura, obtém-se a Figura 3.9. Dessa figura é possível ver que a energia cinética total é mais importante que a energia termobarica. Para modos com número de onda zonal $s<50$, a energia cinética zonal cresce, enquanto a energia termobarica diminui com o aumento de s. Para os modos com número de onda $s>50$ a energia cinética zonal passa a decrescer e a energia termobarica passa a crescer com o aumento de $s$, até que a partir do número de onda $s=400$, a energia termobarica torna-se mais importante que a energia cinética zonal, mas como a componente vertical da energia cinética aumenta, então a energia cinética total mantém-se constante e maior que a energia termobarica.

É interessante analisar pelo menos um modo com índice meridional maior que $l=0$, ou seja, um modo que não seja o de Kelvin. Foi escolhido o modo com o terceiro índice meridional $l=2$. Para os modos acústico-inerciais os gráficos com as energias apresentaram essencialmente o mesmo comportamento. A única diferença foi para a magnitude das energias. As energias dos modos com $l=0$ foram em torno de duas ordens de grandeza maiores que as energias dos modos com índice meridional $l=2$. No entanto, houveram algumas diferenças para os modos gravito-inerciais com número de onda $s<50$.

Os modos gravito-inerciais com índice meridional $l=2$ também apresentaram energia total praticamente constante em relação ao número de onda zonal. Portanto será apresentado apenas o gráfico com as energias normalizadas. Na Figura 3.10 é possível ver 


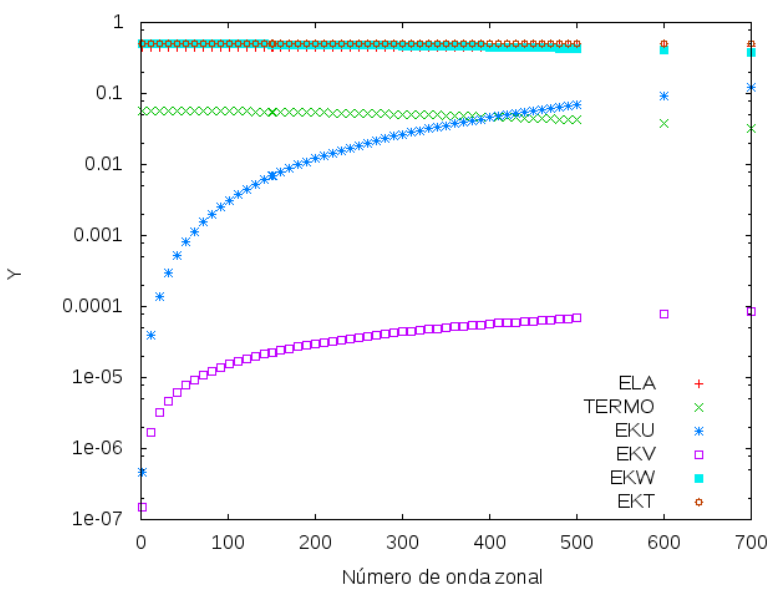

Figura 3.6: Energética dos modos acústico-inerciais com índice meridional $l=0$ e modo vertical $k=1$, onde ET, ELA, TERMO,EKU, EKV, EKW e EKT correspondem, respectivamente às energias elástica, termobarica, cinética zonal, cinética meridional, cinética vertical e cinética total, divididas pela energia total de seu respectivo modo.

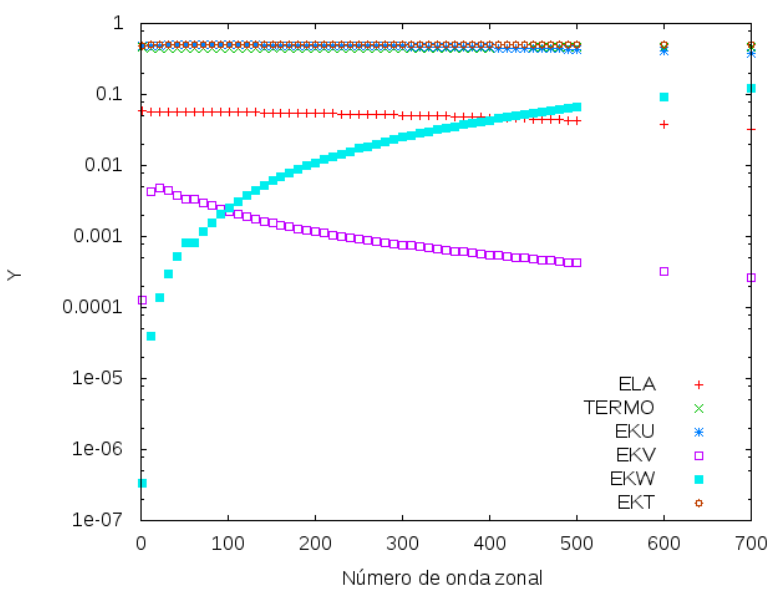

Figura 3.7: Energética dos modos gravito-inerciais com índice meridional $l=0$ e modo vertical $k=1$, onde $E T, E L A, T E R M O, E K U, E K V, E K W$ e $E K T$ correspondem, respectivamente às energias elástica, termobarica, cinética zonal, cinética meridional, cinética vertical e cinética total, divididas pela energia total de seu respectivo modo.

que para o primeiro número de onda zonal $s=1$, a componente meridional da energia cinética é a mais importante. Entretanto, a mesma diminui rapidamente, de tal maneira a torna-se menor que as energias termobarica e cinética zonal para o número de onda zonal $s=10$. Para o modo com número de onda $s=40$, a energia cinética meridional torna-se menos importante que a energia elástica. A energia cinética total apresenta um comportamento semelhante ao comportamento da energia cinética meridional, ou seja, para $s=1$ a energia total é alta, mas decresce rapidamente até o número de onda $s=40$, a partir 


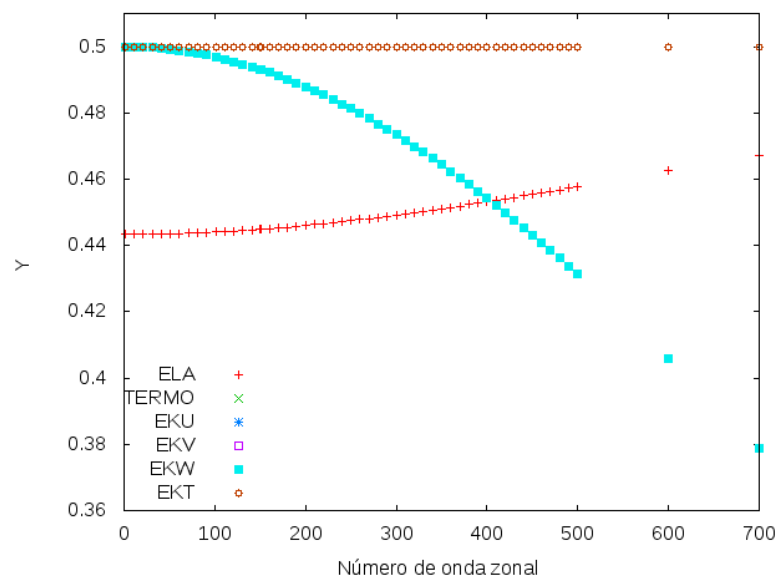

Figura 3.8: O mesmo gráfico da Figura 3.6. mas com o eixo das ordenadas variando entre $[0.36,0.51]$.

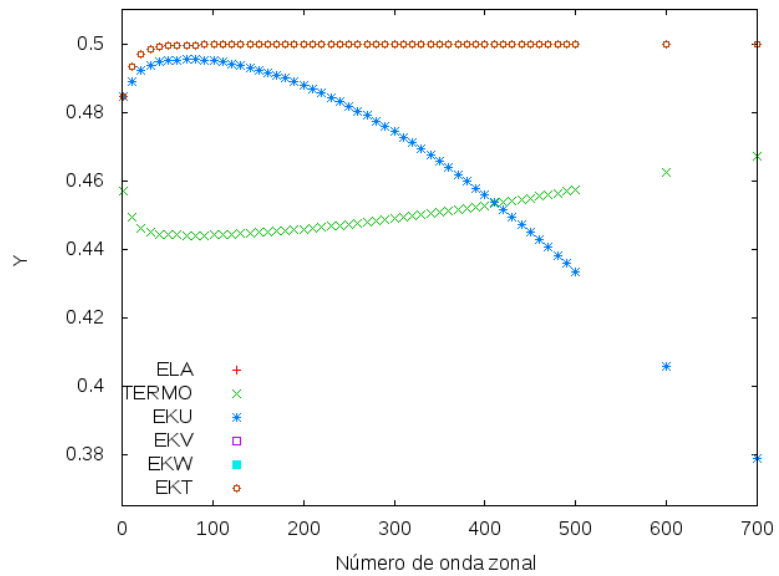

Figura 3.9: O mesmo gráfico da Figura 3.7, mas com o eixo das ordenadas variando entre [0.36, 0.51].

do qual torna-se constante. A energia cinética zonal é ligeiramente menor que a energia termobarica para o primeiro número de onda $s=1$. Porém, para os primeiros números de onda zonal a energia termobarica aumenta mais rápido que a energia cinética zonal, mas a partir do modo com número de onda $s=20$, o crescimento da energia termobarica diminui drasticamente, tal que a energia cinética zonal ultrapassa a energia termobarica em $s=40$. Ainda para os primeiros números de onda zonais, a energia elástica diminui com o aumento de $s$, enquanto para o para o modo de Kelvin a energia elástica aumenta com o aumento de $s$. No entanto, a partir do número de onda zonal $s=40$, o comportamento das energias dos modos com índice meridional $l>0$ é o mesmo que o apresentado pelos modos de Kelvin. 


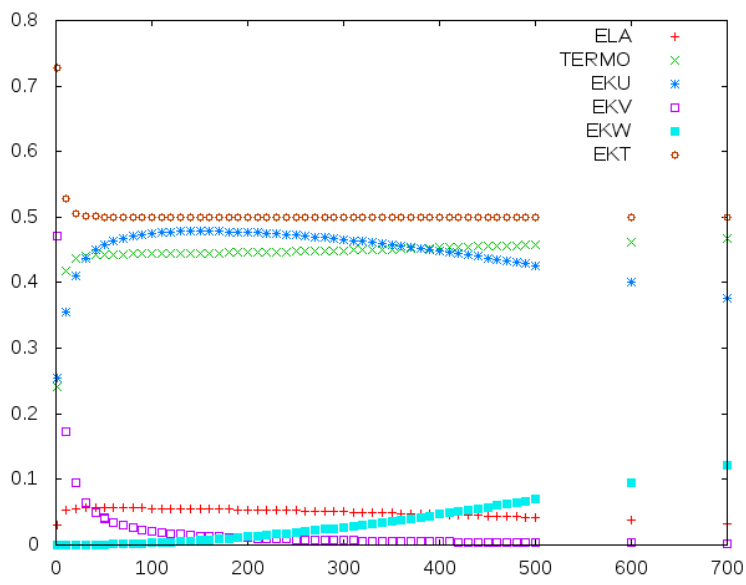

Figura 3.10: O mesmo gráfico da Figura 3.7. mas os modos possuem índice meridional $l=2$ e o eixo das ordenadas não está em escala logarítmica.

\subsubsection{Comparação com a Energética dos Modos Hidrostáticos}

A primeira grande diferença entre os casos hidrostático e não-hidrostático, está na presença de modos acústicos no modelo não-hidrostático. Para comparar a energética dos dois modelos, a soma das energias elástica e termobarica será considerada como a energia potencial disponível (Kasahara, 2004). Kasahara (1976) mostrou que, para os modos de Kelvin, a energia cinética zonal aumenta ao aumentar o número de onda zonal; a energia cinética meridional é muito pequena; e a energia potencial disponível diminui com o aumento do número de onda zonal, tendendo à um valor contante. Além disso, para os outros modos gravito-inerciais de oeste, com índice meridional $l>0$, a energia cinética zonal aumenta, enquanto a energia cinética meridional diminui com o aumento do número de onda zonal; e a energia potencial disponível aumenta com o aumento do número de onda zonal, tendendo à um valor constante. Para os modos normais do modelo não-hidrostático apresentados na Seção 3.2 nota-se que para os modos de Kelvin, a energia cinética zonal inicialmente aumenta com o aumento do número de onda zonal, mas para $s>80$, a energia cinética zonal decresce com o aumento do número de onda zonal; a energia cinética meridional apresenta um comportamento diferente do caso hidrostático, mas seu valor continua sendo muito pequeno, que é a principal característica dos modos de Kelvin; a energia potencial disponível decresce com aumento do número de onda, para os primeiros números de onda zonal, mas para $s>80$ a energia potencial disponível passa a aumentar com o aumento do número de onda zonal. Para os modos com índice meridional $l>0$, a energia 
cinética zonal apresentou o mesmo comportamento que o modo de Kelvin não-hidrostático; a energia cinética meridional apresentou o mesmo comportamento que os modos do modelo hidrostático, com índice meridional $l>0$; a energia elástica apresenta uma leve diminuição com o aumento de $s$, enquanto a energia termobarica apresenta um leve aumento com o aumento de $s$. É preciso fazer o calculo exato da energia potencial disponível para este caso, entretanto esse comportamento antagônico entre a energia elástica e termobarica indica que o comportamento da energia potencial disponível é semelhante ao comportamento do seu correspondente hidrostático.

\subsubsection{Resumo sobre a Energética Linear}

De acordo com os resultados vimos que a energia dos modos acústico-inerciais está concentrada nas energias cinética vertical e na energia elástica. Além disso, a energia cinética vertical aumenta, enquanto a energia elástica diminui, com o aumento do número de onda zonal s. A energética dos modos gravito-inerciais no caso não-hidrostático apresentou muita semelhança com a energética dos modos gravito-inerciais no caso hidrostático. A diferença entre os dois casos começam a aparecer para modos com número de onda zonal maiores, ou seja, para modos mais curtos. Este último resultado era esperado, pois a relação de dispersão dos modos gravito-inerciais não-hidrostáticos diferencia-se do caso hidrostático, apenas para os modos mais curtos, onde o módulo da frequência dessas ondas é limitada superiormente pela frequência de Brunt-Väisälä (Kasahara e Qian, 2000). Um aspecto importante acerca da energética dos modos de gravidade é que a energia cinética vertical aumenta consideravelmente com o aumento do número de onda zonal $s$, tornandose da mesma ordem de magnitude das energias termobárica e cinética zonal para $s>450$. 
Capítulo 4

\section{Energética Não-Linear do Modos Normais}

No capítulo 3 apresentou-se a análise das soluções características do modelo não hidrostático raso. Uma maneira de ampliar o conhecimento sobre a energética do modelo é considerando as interações fracamente não-lineares entre os modos normais do mesmo. Nas teorias fracamente não-lineares assume-se que as soluções características (i.e., as soluções do sistema de equações linearizado) são as soluções em primeira ordem e os efeitos nãolineares são fenômenos de ordem superior. No presente capítulo serão considerados os efeitos não-lineares de ordem quadrática em relação as variáveis perturbadas.

No que se segue, será analisada a dinâmica das interações fracamente não-lineares entre os modos normais do modelo não-hidrostático raso, representado pelas equações (2.4)-(2.9), com o intuito de estender a teoria linear dos modos normais não-hidrostáticos desenvolvida por Kasahara e Qian (2000). Para tal propósito, o sistema de equações (2.30), que contém as não-linearidades dominantes (quadráticas) do sistema original, será analisado através de uma expansão de Galerkin usando como funções base as próprias soluções características do sistema linearizado, pois como foi mostrado na seção anterior, estas soluções características constituem uma base ortogonal. Mais especificamente será analisada as trocas de energia entres modos que compõem um tripleto ressonante.

\subsection{Ansatz - Expansão de Galerkin}

Considere o seguinte ansatz para a solução do sistema 2.30 


$$
\vec{u}=\left[\begin{array}{c}
u^{\prime} \\
v^{\prime} \\
w^{\prime} \\
p^{\prime} \\
\theta^{\prime}
\end{array}\right]=\sum_{b} A_{b}(t)\left[\begin{array}{ccccc}
\rho_{0}^{-1 / 2} & 0 & 0 & 0 & 0 \\
0 & \rho_{0}^{-1 / 2} & 0 & 0 & 0 \\
0 & 0 & \rho_{0}^{-1 / 2} & 0 & 0 \\
0 & 0 & 0 & \rho_{0}^{+1 / 2} & 0 \\
0 & 0 & 0 & 0 & \rho_{0}^{+1 / 2}
\end{array}\right] \vec{\Lambda}_{b}(\phi, z) \exp i\left(s_{b} \lambda-\sigma_{b} t\right)
$$

onde $\vec{\Lambda}_{b}=\left[U_{b}, i V_{b}, i W_{b}, P_{b}, \theta_{b}\right]^{T}$ é o vetor que descreve as estruturas meridional e vertical de um dado modo, ou seja, $\vec{\Lambda}_{b}$ satisfaz (3.3)-(3.7), com condições de contorno do tipo tampa rígida na vertical, periodicidade zonal e regularidade nos polos, e para $\sigma=\sigma_{b}$ e $s=s_{b}$, onde o índice $b=(s, l, k, r)$ representa um modo normal qualquer, onde $s$ é o número de onda zonal, $l$ representa o modo meridional, $k$ é o número de onda vertical e $r$ indica qual o tipo de onda (neste caso, indica se o modo representa uma oscilação de primeira, segunda ou terceira espécie). Assim, $\sum_{b}$ representa o somatório de todos os modos possíveis, sendo $A_{b}(t)$ a amplitude do $b$-ésimo modo. Dessa forma no ansatz acima as variáveis dinâmicas são escritas como uma superposição das autofunções do problema linearizado (3.2), mas com as amplitudes dos modos permitidas a variarem no tempo em função do acoplamento não-linear entre os modos.

Uma vez que as autofunções do problema satisfazem a condição de ortogonalidade (3.81) e essas autofunções são utilizadas como funções base para a expansão de Galerkin, é natural utilizar o seguinte produto interno $\langle\cdot, \cdot\rangle: \mathbb{H} \times \mathbb{H} \rightarrow \mathbb{R}$, onde $\mathbb{H}$ é o espaço de Hilbert formado pelas autofunções do problema linearizado:

$$
\langle\vec{u}, \vec{v}\rangle=\int_{V}\left[\frac{\rho_{0}}{2}\left(u_{1} v_{1}^{*}+u_{2} v_{2}^{*}+u_{3} v_{3}^{*}\right)+\frac{u_{4} v_{4}^{*}}{2 \rho_{0} C_{s}^{2}}+\frac{u_{5} v_{5}^{*}}{2 \rho_{0} N^{2}}\right] d V
$$

onde $\vec{u}=\left[u_{1}, u_{2}, u_{3}, u_{4}, u_{5}\right]^{T}$ e $\vec{v}=\left[v_{1}, v_{2}, v_{3}, v_{4}, v_{5}\right]^{T}$ representam duas funções vetoriais quaisquer de quadrado integrável no domínio $V=\left\{[0,2 \pi] \times[-\pi / 2, \pi / 2] \times\left[0, z_{T}\right]\right\}$.

\subsection{Evolução Temporal das Amplitudes dos Modos e Condição de Ressonância Triádica}

Nesta seção será apresentado o sistema de equações que descreve a evolução temporal da amplitude de cada modo normal do sistema linearizado, mas admitindo as interações 
fracamente não-lineares entre esses modos normais. Majda (2003) apresenta a metodologia empregada nesta seção em detalhes para sistemas dispersivos não-lineares e sob a hipótese WKB.

Substituindo o ansatz (4.1) no sistema (2.30) e projetando o mesmo (com o auxílio do produto interno (4.2)) no a-ésimo modo, obtém-se:

$$
E_{a} \frac{d A_{a}}{d t}=i \sum_{b} \sum_{c} A_{b} A_{c}\left\langle\mathcal{B}, \vec{\Lambda}_{a}\right\rangle
$$

onde $E_{a}$ refere-se à energia linear do modo $a$ dada por $(3.82)-(3.85), \mathcal{B}$ é o vetor com os termos não-lineares 2.31, mas com as derivadas com relação à longitude sendo substituídas por is, ou seja,

$$
\mathcal{B}(\vec{u}, \vec{u})=\left[\mathcal{B}^{(1)}, \mathcal{B}^{(2)}, \mathcal{B}^{(3)}, \mathcal{B}^{(4)}, \mathcal{B}^{(5)}\right]^{T} \exp i\left[\left(s_{b}+s_{c}\right) \lambda-\left(\sigma_{b}+\sigma_{c}\right) t\right]+\mathrm{cyc}
$$

onde cyc representa a permutação cíclica em relação aos índices $b$ e $c$ e as componentes $\mathcal{B}^{(1)}, \mathcal{B}^{(2)}, \mathcal{B}^{(3)}, \mathcal{B}^{(4)}, \mathcal{B}^{(5)}$ são dadas pelas seguintes expressões:

$$
\begin{aligned}
\mathcal{B}^{(1)}= & \rho_{0}^{-1 / 2}\left[-\frac{U_{b}}{a \cos \phi} i s_{c} U_{c}-\frac{V_{b}}{a} \frac{\partial U_{c}}{\partial \phi}-W_{b} \frac{\partial U_{c}}{\partial z}-\frac{W_{b} U_{c}}{2 H}+\frac{U_{b} V_{c}}{a} \tan \phi\right] \\
& +\rho_{0}^{-1 / 2}\left[\frac{\rho_{b}}{a \cos \phi} i s_{c} P_{c}\right] \\
\mathcal{B}^{(2)}= & -\rho_{0}^{-1 / 2}\left(-\frac{U_{b}}{a \cos \phi} i s_{c} V_{c}-\frac{V_{b}}{a} \frac{\partial V_{c}}{\partial \phi}-W_{b} \frac{\partial V_{c}}{\partial z}-\frac{W_{b} V_{c}}{2 H}+\frac{U_{b} U_{c}}{a} \tan \phi\right) \\
& -\rho_{0}^{-1 / 2}\left(-\frac{\rho_{b}}{a} \frac{\partial P_{c}}{\partial \phi}\right) \\
\mathcal{B}^{(3)}= & -\rho_{0}^{-1 / 2}\left(-\frac{U_{b}}{a \cos \phi} i s_{c} W_{c}-\frac{V_{b}}{a} \frac{\partial W_{c}}{\partial \phi}-W_{b} \frac{\partial W_{c}}{\partial z}-\frac{W_{b} W_{c}}{2 H}-\rho_{b} \frac{\partial P_{c}}{\partial z}\right) \\
& -\rho_{0}^{-1 / 2}\left(\frac{\rho_{b} P_{c}}{2 H}\right)+g \rho_{0}^{-1 / 2} \rho_{b} \rho_{c} \\
\mathcal{B}^{(4)}= & \rho_{0}^{-1 / 2}\left[-\frac{1}{C_{s}^{2}}\left(\frac{U_{b}}{a \cos \phi} i s_{c} P_{c}+\frac{1}{a} V_{b} \frac{\partial P_{c}}{\partial \phi}+W_{b} \frac{\partial P_{c}}{\partial z}-\frac{W_{b} P_{c}}{2 H}\right)\right] \\
& +\rho_{0}^{-1 / 2}\left[-\frac{1}{a \cos \phi}\left(\rho_{b} i s_{c} U_{c}+\rho_{b} \cos \phi \frac{\partial V_{c}}{\partial \phi}-\rho_{b} V_{c} \sin \phi\right)\right] \\
& +\rho_{0}^{-1 / 2}\left[-\rho_{b} \frac{\partial W_{c}}{\partial z}-\frac{\rho_{b} W_{c}}{2 H}-\frac{1}{C_{s}^{2}} \frac{\rho_{0} T_{b}}{T_{0}}\left(\sigma_{c} P_{c}+g W_{c}\right)\right]
\end{aligned}
$$




$$
\begin{aligned}
\mathcal{B}^{(5)}= & \rho_{0}^{-1 / 2}\left[-\frac{U_{b}}{a \cos \phi} i s_{c} \theta_{c}-\frac{V_{b}}{a} \frac{\partial \theta_{c}}{\partial \phi}-W_{b} \frac{\partial \theta_{c}}{\partial \phi}-W_{b} \frac{\partial \theta_{c}}{\partial z}+\frac{W_{b} \theta_{c}}{2 H}\right] \\
& -\rho_{0}^{-1 / 2}\left[\frac{g}{C_{s}^{2}} \frac{\rho_{0} T_{b}}{T_{0}}\left(\sigma_{c} P_{c}+g W_{c}\right)\right]
\end{aligned}
$$

A equação (4.3) mostra que o efeito da não-linearidade é quebrar a independência dos modos, onde a interação se dá por tripletos devido à natureza quadrática dos termos nãolineares. A evolução da amplitude do modo $a$ é modificada pela interação de vários modos $b$ e $c$. Porém, analisando o termo $\left\langle\mathcal{B}, \vec{\Lambda}_{a}\right\rangle$ é possível inferir que existem certos modos $b$ e $c$ que interagem de forma mais significativa com o modo $a$, que são os modos que satisfazem certas condições de ressonância com o modo $a$.

As condições de ressonância estão associadas às condições para que o valor do produto interno $\left\langle\mathcal{B}, \vec{\Lambda}_{a}\right\rangle$ seja máximo. Na longitude tem-se a seguinte integral

$$
I_{\lambda}=\int_{0}^{2 \pi} \exp \left[i\left(s_{b}+s_{c}-s_{a}\right) \lambda\right] d \lambda
$$

mas, devido ao comportamento oscilatório da exponencial com argumento complexo, é fácil ver que a integral acima é diferente de zero apenas quando $s_{b}+s_{c}-s_{a}=0$.

A condição para a integral na latitude é que a estrutura meridional do integrando do produto interno em (4.3) seja simétrica em relação ao equador, pois no caso de uma estrutura antissimétrica a integral relacionada à projeção do termo não-linear em um dado modo é nula. Logo, para que exista interação é necessário que o tripleto seja constituído por três modos simétricos ou por dois antissimétricos e um simétrico.

A integral na vertical possui termos da seguinte forma:

$$
I_{z}=\int_{0}^{z_{T}} \rho_{0}^{ \pm 1 / 2} \exp \left[i\left(k_{b}+k_{c}-k_{a}\right)\right] d z
$$

Como a função $\rho_{0}(z)$ é positivo definida $\left(\rho_{0}(z)=\left.\rho_{0}\right|_{z=0} \exp (-z / H)\right)$, então para $k_{b}+$ $k_{c}-k_{a} \neq 0$ o comportamento oscilatório da exponencial complexa irá diminuir o valor da integral. Dessa forma, a integral na vertical $I_{z}$ terá máxima contribuição quando $k_{b}+k_{c}-$ $k_{a}=0$. Vale notar que essa condição de ressonância não é excludente, ou seja, podem existir tripletos ressonantes que não satisfazem essa condição. Porém, a interação entres os modos que compõem tais tripletos deve ser mais fraca do que as interações num tripleto que satisfaça tal condição. 
Para resolver o sistema de equações diferenciais ordinárias para as amplitudes, a seguinte integral deve ser resolvida:

$$
I_{t} \sim \int_{t_{0}}^{t_{1}} f(t) \exp \left[i\left(\sigma_{b}+\sigma_{c}-\sigma_{a}\right) t\right] d t
$$

onde $f(t)$ representa o produto das amplitudes de dois modos $b$ e $c$ quaisquer. Pelo princípio da fase estacionária (Majda, 2003), se $f(t)$ variar numa escala mais lenta comparada com a escala de variação da fase da exponencial complexa, a integral acima é assintoticamente pequena se a fase não for estacionária, ou seja, a integral é dominada pelos pontos no espaço de frequências onde $\sigma_{b}+\sigma_{c}-\sigma_{a}=0$. Essa condição dificilmente é satisfeita, por isso serão considerados tripletos que satisfaçam essa condição apenas de forma aproximada, ou seja, $\sigma_{b}+\sigma_{c}-\sigma_{a} \approx 0$. A hipótese da amplitude dos modos variando numa escala lenta comparada com a variação temporal da fase dos mesmos está intimamente ligada à hipótese de pequenas amplitudes (Raupp et al., 2008) e é completamente satisfeita nos experimentos numéricos apresentados nesta seção, como mostrado mais adiante.

Com isso, no regime de pequenas amplitudes, somente os modos $b$ e $c$ que satisfazem as seguintes condições de ressonância com o modo $a$

$$
\begin{gathered}
\sigma_{a}=\sigma_{b}+\sigma_{c} \\
s_{a}=s_{b}+s_{c} \\
k_{a}=k_{b}+k_{c}
\end{gathered}
$$

As condições 4.13)- 4.15 somada à condição de simetria meridional mencionada anteriormente, contribuem de forma expressiva para a evolução não-linear do modo $a$. Os modos $b, c$ e $a$ que satisfazem às condições acima constituem um tripleto ressonante.

\subsection{Evolução Temporal para um Tripleto Ressonante}

O sistema 4.3 descreve a evolução temporal de todos os modos normais sujeitos às interações fracamente não-lineares. Para analisar isoladamente a interação de apenas um tripleto ressonante, é preciso truncar a expansão (4.1) para um conjunto de três modos $b$, $c$ e $a$ que satisfazem às condições de ressonância enumeradas acima. Neste caso, o sistema de equações (4.3) reduz-se à: 


$$
\begin{aligned}
& E_{a} \frac{d A_{a}}{d t}=i \alpha_{a}^{b c} A_{b} A_{c} \\
& E_{b} \frac{d A_{b}}{d t}=i \alpha_{b}^{c a} A_{a} A_{c}^{*} \\
& E_{c} \frac{d A_{c}}{d t}=i \alpha_{c}^{a b} A_{a} A_{b}^{*}
\end{aligned}
$$

onde o símbolo $*$ nas equações acima indicam o complexo conjugado das amplitudes espectrais e $E_{b}, E_{c}$ e $E_{a}$ correspondem à norma quadrática dos modos $b, c$ e $a$, respectivamente, dadas por (3.82)-3.85). Note que no caso linear, onde as amplitudes $A_{a}, A_{b}$ e $A_{c}$ não variam no tempo, $E_{a}, E_{b}$ e $E_{c}$ representam as próprias energias dos modos. Os coeficientes $\alpha_{a}^{b c}, \alpha_{b}^{c a}$ e $\alpha_{c}^{b a}$ em 4.16)-4.18 são os coeficientes de interação, expressos da seguinte forma:

$$
\alpha_{a}^{b c}=\left\langle\mathcal{B}\left(\vec{\Lambda}_{b}, \vec{\Lambda}_{c}\right), \vec{\Lambda}_{a}\right\rangle
$$

O coeficiente $\alpha_{a}^{b c}$ representa a influência que a interação entre os modos $b$ e $c$ tem sob o modo a. Além disso, o valor do coeficiente $\alpha_{a}^{b c}$ indica o quão forte é essa influência Craik (1988). O sistema de equações (4.16)-4.18) é um sistema bem conhecido e já foi estudado em vários outros contextos como nas áreas de eletrônica, física de plasma, ótica e mecânica de fluidos (Craik, 1988). A solução analítica do sistema é dada em termos das funções elípticas de Jacobi (Craik, 1988; Abarbanel e Rabinovich, 1993, Raupp, 2006: Raupp et al. 2008). Considerando que o modo a representa o modo com maior coeficiente de interação (em valores absolutos) e o modo $c$ é o modo com menor coeficiente de interação e além disso supondo que a amplitude inicial do modo $a$ é nula $A_{a}(t=0)=A_{a}^{0}=0$, tem-se que nessas condições o período de troca de energia do tripleto depende de um parâmetro $\tilde{m}$ Raupp et al. 2008):

$$
\tilde{m}=\frac{\alpha_{c}^{a b}}{\alpha_{b}^{c a}} \frac{E_{b}}{E_{c}} \frac{\left(A_{b}^{0}\right)^{2}}{\left(A_{c}^{0}\right)^{2}}
$$

onde $A_{b}^{0}$ e $A_{c}^{0}$ são as amplitudes iniciais dos modos $b$ e $c$.

Entretanto, para analisar as trocas de energia num tripleto ressonante para o modelo aqui estudado é necessário primeiramente obter a lei de conservação da energia total do sistema fracamente nãolinear representado por 2.30. Para tanto, basta projetar o sistema 2.30 no vetor estado $\vec{u}$ : 


$$
\langle\mathcal{L} \vec{u}, \vec{u}\rangle=\langle\mathcal{N}(\vec{u}, \vec{u}), \vec{u}\rangle \Rightarrow \frac{\partial}{\partial t} E T=\mathcal{O}\left(\left(u^{\prime}, v^{\prime}, w^{\prime}, p^{\prime}, \theta^{\prime}, \rho^{\prime}\right)^{3}\right)
$$

onde

$$
E T=\int_{V}\left(\frac{\rho_{0}}{2}\left(u^{\prime 2}+v^{\prime 2}+w^{\prime 2}\right)+\frac{p^{\prime 2}}{\rho_{0} C_{s}^{2}}+\frac{\theta^{\prime 2}}{\rho_{0} N^{2}}\right) d V
$$

Assim, a equação 4.21 mostra que, no regime de pequenas amplitudes, ET representa o termo de ordem dominante da energia total do sistema. Substituindo o ansatz 4.1 na expressão da energia total 4.22, obtém-se a identidade de Parseval para a energia de ordem dominante:

$$
E T=\sum_{b} E_{b}\left|A_{b}\right|^{2}
$$

Portanto a energia total de ordem dominante do sistema é igual a soma do quadrado das amplitudes de cada modo multiplicada por sua respectiva energia $E_{b}$ (do caso linearizado).

Dada a identidade de Parseval (4.23), a partir do sistema 4.16)-(4.18) é possível obter a equação para a evolução temporal da energia de cada modo do tripleto:

$$
\begin{aligned}
& E_{a} \frac{d}{d t}\left|A_{a}\right|^{2}=2 i \alpha_{d}^{b c} \operatorname{Im}\left(A_{b} A_{c} A_{a}^{*}\right) \\
& E_{b} \frac{d}{d t}\left|A_{b}\right|^{2}=-2 i \alpha_{b}^{c a} \operatorname{Im}\left(A_{b} A_{c} A_{a}^{*}\right) \\
& E_{c} \frac{d}{d t}\left|A_{c}\right|^{2}=-2 i \alpha_{c}^{a b} \operatorname{Im}\left(A_{b} A_{c} A_{a}^{*}\right)
\end{aligned}
$$

onde o símbolo Im indica a parte imaginária de seu argumento. Do sistema de equações acima é possível ver que a energia total do tripleto será conservada se os coeficientes de interação $\alpha_{a}^{b c}, \alpha_{b}^{c a}$ e $\alpha_{c}^{a b}$ satisfizerem a seguinte condição:

$$
\alpha_{a}^{b c}=\alpha_{b}^{c a}+\alpha_{c}^{a b}
$$

\subsection{Determinação de um Tripleto Ressonante}

Kasahara e Qian (2000) mostraram que no modelo descrito pelas equações (2.4)-(2.9): os modos rotacionais (i.e., modos de baixa frequência) praticamente não são afetados pelos 
efeitos não hidrostáticos; apenas os modos gravito-inerciais mais curtos, i.e., com números de onda zonal $s>400$, são afetados de forma significativa pelos efeitos não hidrostáticos; e o abandono da aproximação hidrostática resulta num terceiro tipo de solução característica do modelo, os modos acústico-inerciais. Portanto, para estudar tripletos exclusivos da versão não hidrostática do modelo, será apresentado um estudo sobre as trocas de energia entre um modo acústico-inercial de escala planetária, um modo acústico inercial de pequena escala espacial $(s>400)$ e um modo gravito-inercial também de pequena escala espacial $(s>400)$.

Para determinar um tripleto ressonante composto por dois modos acústico-inerciais e um modo gravito-inercial utilizou-se um método gráfico. Primeiramente foram obtidas as curvas de dispersão dos modos acústico-inerciais com índice meridional $l=0$, modo vertical $k=1$ e com estrutura meridional simétrica e dos modos gravito-inerciais com índice meridional $l=0$, modo vertical $k=2$ e com estrutura meridional simétrica. Dessa forma, todos os modos da Figura 4.1a satisfazem as condições de ressonância meridional e vertical. Dentre esses modos, é preciso determinar quais deles satisfazem as condições de ressonância no número de onda zonal (4.14) e na frequência temporal (4.13). Para determinar esses modos deslocamos a origem da relação de dispersão dos modos gravitoinerciais num ponto da relação de dispersão dos modos acústico-inerciais (Figura 4.1b).

Em posse da Figura 4.1b, basta verificar onde as duas curvas se intersectam. O primeiro ponto de intersecção, onde é colocada a origem da curva de dispersão dos modos gravito-inerciais, já corresponde ao primeiro modo do tripleto ressonante, que neste caso corresponde a um modo acústico-inercial longo $(s=1)$. Através do segundo ponto de intersecção nós obtemos os outros dois modos que satisfazem às condições (4.13) e 4.14), sendo o primeiro modo um modo acústico-inercial curto e o segundo um modo gravitoinercial curto. Assim, utilizando este método gráfico determinou-se um tripleto composto por um modo acústico-inercial com número de onda zonal $s=476$, índice meridional $l=0$ e modo vertical $k=1$ (modo $a$ ), outro modo acústico-inercial com número de onda zonal $s=1$, índice meridional $l=0$ e modo vertical $k=1$ (modo $b$ ) e um modo gravito-inercial com número de onda zonal $s=475$, índice meridional $l=0$ e modo vertical $k=2$ (modo c) . Além disso a frequência desses modos são dadas por $\sigma_{a}=6.293 \mathrm{cHz}, \sigma_{b}=5.888 \mathrm{cHz}$ e $\sigma_{c}=0.407 \mathrm{cHz}$, respectivamente. 


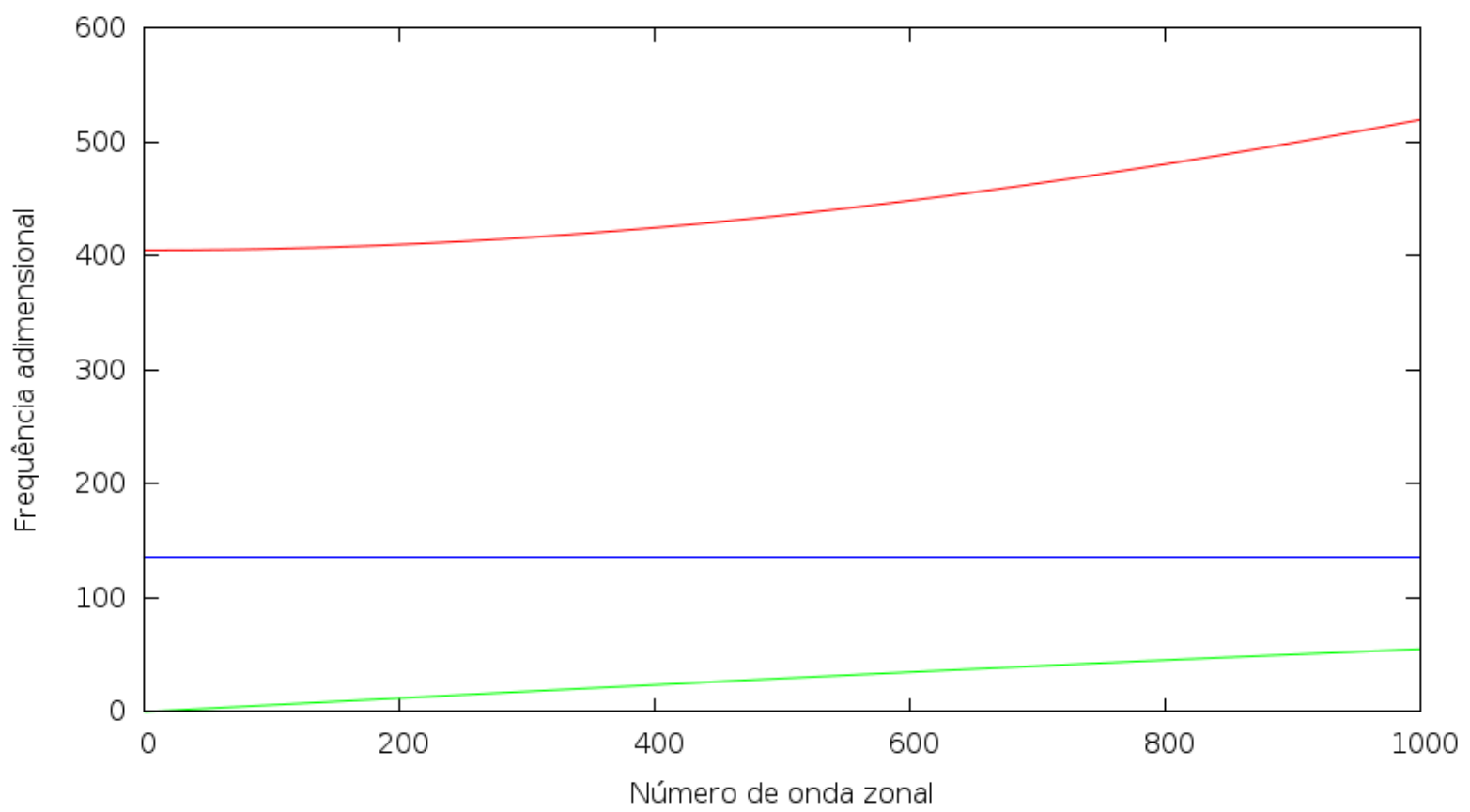

(a)

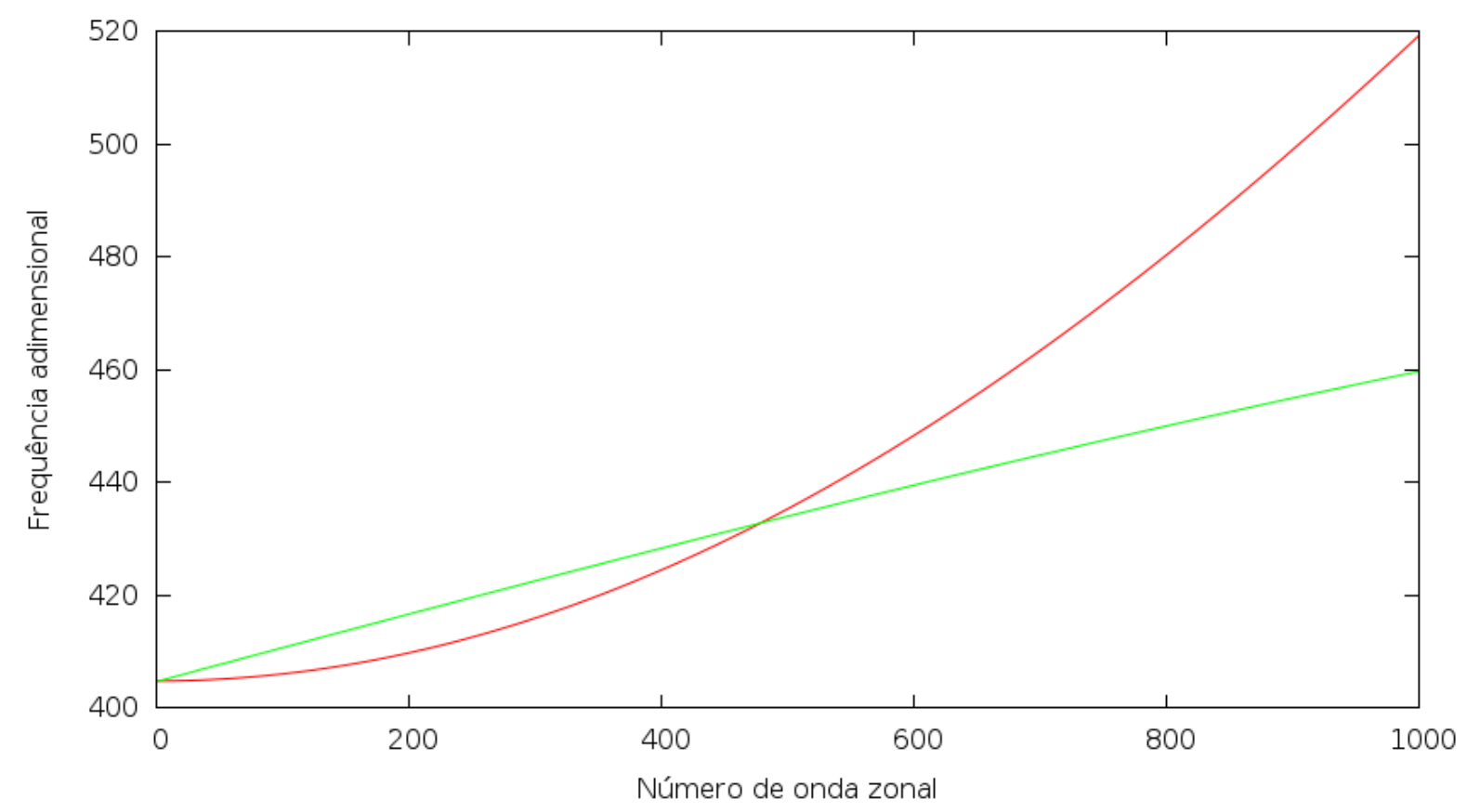

(b)

Figura 4.1: As figuras acima são apenas diagramas representando método utilizado para obter os tripletos ressonantes. Os valores nos eixos são apenas ilustrativos. Na figura 4.1a temos a relação de dispersão dos modos gravito-inerciais (curva verde) e dos modos acústico-inerciais (curva vermelha) . A curva $N$ representa a frequência de Brunt-Väisälä (curva azul), que separa dos dois regimes citados. Na figura $4.1 b$ temos a mesma figura, mas agora a origem da relação de dispersão dos modos acústico inerciais está deslocada para cima. 


\subsection{Algumas soluções particulares para a evolução temporal de um} tripleto

O sistema de equações 4.16, 4.17 e 4.18) apresenta algumas soluções particulares que ajudam a elucidar a dinâmica das trocas de energia num tripleto ressonante. Primeiramente será analisada a estabilidade de apenas um dos modos $A_{1}$, quando a amplitude dos outros modos $A_{2}$ e $A_{3}$ são pequenas quando comparadas com $A_{1}$. Esta primeira análise pode ser considerada como a analise da estabilidade linear do sistema de equações considerado Drazin e Reid (1981). Posteriormente, será considerado o caso em que as amplitudes $A_{i}, i=1,2,3$ são reais e apresentam um comportamento periódico.

\subsubsection{Estabilidade linear de apenas um modo}

Para facilitar as manipulações matemática a seguir, o sistema 4.16), 4.17) e (4.18) será reescrito da seguinte forma:

$$
\begin{aligned}
& E_{1} \frac{d A_{1}}{d t}=i \alpha_{1} A_{2} A_{3} \\
& E_{2} \frac{d A_{2}}{d t}=i \alpha_{2} A_{1} A_{3}^{*} \\
& E_{3} \frac{d A_{3}}{d t}=i \alpha_{3} A_{1} A_{2}^{*}
\end{aligned}
$$

onde $A_{1}=A_{a}, A_{2}=A_{b}$ e $A_{3}=A_{c} ; E_{1}=E_{a}, E_{2}=E_{b}$ e $E_{3}=E_{c} ;$ e o coeficientes $\alpha_{1}$, $\alpha_{2}$ e $\alpha_{3}$ são os coeficientes $\alpha_{a}^{b c}, \alpha_{b}^{c a}$ e $\alpha_{c}^{a b}$, respectivamente.

Assumindo que as amplitudes $A_{2}$ e $A_{3}$ são muito menores que a amplitude $A_{1}$ e que a amplitude $A_{1} \neq 0$ é constante, as equações 4.28, 4.29) e 4.30 podem ser reescritas da seguinte forma:

$$
\begin{aligned}
\frac{d A_{1}}{d t} & =0 \\
E_{2} \frac{d A_{2}}{d t} & =i \alpha_{2} A_{1} A_{3}^{*} \\
E_{3} \frac{d A_{3}}{d t} & =i \alpha_{3} A_{1} A_{2}^{*} \\
E_{2} \frac{d A_{2}^{*}}{d t} & =-i \alpha_{2} A_{1}^{*} A_{3} \\
E_{2} \frac{d A_{3}^{*}}{d t} & =-i \alpha_{3} A_{1}^{*} A_{2}
\end{aligned}
$$


O sistema de equações acima pode ser facilmente reduzido à um sistema de duas equações diferenciais de segunda ordem:

$$
\begin{aligned}
\frac{d^{2} A_{2}}{d t^{2}} & =\frac{\alpha_{2} \alpha_{3}}{E_{2} E_{3}}\left|A_{1}\right|^{2} A_{2} \\
\frac{d^{2} A_{3}}{d t^{2}} & =\frac{\alpha_{2} \alpha_{3}}{E_{2} E_{3}}\left|A_{1}\right|^{2} A_{3}
\end{aligned}
$$

Desta forma torna-se evidente que $A_{2}$ e $A_{3}$ podem apresentar um comportamento periódico ou um crescimento exponencial. Como $E_{i}>0, i=1,2,3$ e $\left|A_{1}\right|>0$, então as amplitudes $A_{i}, i=2,3$ apresentarão comportamento periódico se $\alpha_{2} \alpha_{3}<0$. Por outro lado, se $\alpha_{2} \alpha_{3}>0$, então as amplitudes $A_{i}, i=2,3$ apresentarão um crescimento exponencial. Tratando-se de tripletos conservativos, ou seja, tripletos cujos coeficientes de interação satisfazem a relação $\alpha_{1}=\alpha_{2}+\alpha_{3}$, a análise da estabilidade linear de um modo garante que sempre haverá o crescimento exponencial dos modos $A_{i}, i=2,3$.

\subsubsection{Solução particular para amplitudes reais}

Nesta seção será demonstrada explicitamente que, para um caso particular, a solução do sistema dinâmico que descreve a evolução temporal de um tripleto ressonante é dada em termos das funções elípticas de Jacobi.

Utilizando a seguinte substituição de variáveis

$$
A_{j} \rightarrow \frac{A_{j}}{\sqrt{\alpha_{k} \alpha_{l}}}
$$

onde os índices $j, k$ e $l$ podem assumir os valores 1,2 e 3 . Dessa forma o sistema de equações 4.28, 4.29) e 4.30 pode ser reescrito como

$$
\begin{aligned}
& \frac{d A_{1}}{d t}=i \sigma_{1} A_{2} A_{3} \\
& \frac{d A_{2}}{d t}=i \sigma_{2} A_{1} A_{3}^{*} \\
& \frac{d A_{3}}{d t}=i \sigma_{3} A_{1} A_{2}^{*}
\end{aligned}
$$

onde o coeficiente $\sigma_{j}$ é definido por 


$$
\sigma_{j}=\frac{\alpha_{j} / E_{j}}{\left|\alpha_{j} / E_{j}\right|} \equiv \operatorname{sign}\left(\frac{\alpha_{j}}{E_{j}}\right)
$$

Como as amplitudes $A_{j}$ são variáveis complexas, então elas podem ser escritas na forma polar como

$$
A_{j}(t)=b_{j}(t) \exp \eta_{j}(t)
$$

onde $b_{j}(t)=\left|A_{j}(t)\right|$ e $\eta_{j}(t)$ é a fase da variável complexa. Na forma polar, o sistema de equações acima pode ser reescrito como

$$
\begin{aligned}
\frac{d b_{1}}{d t} & =\sigma_{1} b_{2} b_{3} \sin \eta \\
\frac{d b_{2}}{d t} & =-\sigma_{2} b_{1} b_{3} \sin \eta \\
\frac{d b_{3}}{d t} & =-\sigma_{3} b_{1} b_{2} \sin \eta \\
\frac{d \eta}{d t} & =b_{1} b_{2} b_{3}\left(\frac{\sigma_{1}}{b_{1}^{2}}+\frac{\sigma_{2}}{b_{2}^{2}}+\frac{\sigma_{3}}{b_{3}^{2}}\right)
\end{aligned}
$$

As soluções deste sistema de equações são dadas em termos das funções elípticas de Jacobi e as mesmas podem ser encontradas em Craik (1988). Nesta seção será considerado o caso em que a fase $\eta$ é constante para ilustrar o método de obtenção da solução analítica da evolução temporal de um dos modos que compõe um tripleto ressonante. No caso em que a fase $\eta$ é constante, o sistema de equações acima resulta nas equações de Euler do corpo rígido na ausência de torque, cuja solução é bem conhecida (Landau e Lifshitz, 1976).

Para o caso em que a fase $\eta$ é constante será considerado que, sem perda de generalidade, $\eta=\pi / 2$. Além disso, será considerado que $\sigma_{i}>0, i=1,2,3$ (Craik, 1988). Portanto, o sistema de equações acima torna-se

$$
\begin{aligned}
& \frac{d b_{1}}{d t}=\sigma_{1} b_{2} b_{3} \\
& \frac{d b_{2}}{d t}=-\sigma_{2} b_{1} b_{3} \\
& \frac{d b_{3}}{d t}=-\sigma_{3} b_{1} b_{2}
\end{aligned}
$$


É possível mostrar que o sistema de equações acima apresenta as seguintes quantidades conservativas (i.e., relações de Manley-Rowe (Craik, 1988))

$$
\begin{aligned}
& \Omega_{1}:=\sigma_{1} b_{1}^{2}+b_{2}^{2} \\
& \Omega_{2}:=\sigma_{1} b_{1}^{2}+\sigma_{3} b_{3}^{2} \\
& \Omega_{3}:=\sigma_{2} b_{2}^{2}-\sigma_{3} b_{3}^{2}
\end{aligned}
$$

Com as equações 4.51) e 4.53 é possível mostrar que

$$
\begin{gathered}
\sigma_{1} b_{1}^{2}=\Omega_{1}-\sigma_{2} b_{2}^{2} \\
\sigma_{3} b_{3}^{2}=-\Omega_{3}+\sigma_{2} b_{2}^{2}
\end{gathered}
$$

Substituindo 4.54) e 4.55) em 4.49), obtém-se

$$
\frac{d b_{2}}{d t}=-\sigma_{2}\left(\frac{\Omega_{1}-\sigma_{2} b_{2}^{2}}{\sigma_{1}}\right)^{1 / 2}\left(\frac{\sigma_{2} b_{2}^{2}-\Omega_{3}}{\sigma_{3}}\right)^{1 / 2}
$$

Utilizando a seguinte substituição de variáveis

$$
\begin{aligned}
& \tau=t \sigma_{2} \sqrt{\frac{\Omega_{3} \sigma_{2}}{\sigma_{1} \sigma_{3}}} \\
& s=b_{2} \sqrt{\frac{\sigma_{2}}{\Omega_{1}}}
\end{aligned}
$$

e definindo um parâmetro positivo $k^{2}<1$ (Whittaker e Watson, 1950; Landau e Lifshitz, 1976)

$$
k^{2}=\frac{\Omega_{1}}{\Omega_{3}}
$$

Com isso, a equação 4.56 torna-se

$$
\frac{d s}{d \tau}=\sqrt{\left(1-s^{2}\right)\left(1-k^{2} s^{2}\right)}
$$

A equação acima pode ser escrita na forma da seguinte integral 


$$
\tau=\int_{0}^{s} \frac{d s}{\sqrt{\left(1-t^{2}\right)\left(1-k^{2} t^{2}\right)}}
$$

Com isso, $s=\operatorname{sn}(\tau, k)$, onde a função sn é o conhecida como seno elíptico de Jacobi. Portanto, com o auxílio das equações 4.54 e 4.53), a solução do sistema que descreve a evolução temporal das amplitudes de um tripleto ressonante, quando $\sigma_{1}=\sigma_{2}=\sigma_{3}$ são dadas por

$$
\begin{aligned}
& b_{1}=\sqrt{\frac{\Omega_{1}}{\sigma_{1}}} c n\left(\sqrt{\frac{\Omega_{3} \sigma_{2}}{\sigma_{1} \sigma_{3}}} \sigma_{2} t, k\right) \\
& b_{2}=\sqrt{\frac{\Omega_{1}}{\sigma_{2}}} s n\left(\sqrt{\frac{\Omega_{3} \sigma_{2}}{\sigma_{1} \sigma_{3}}} \sigma_{2} t, k\right) \\
& b_{3}=\sqrt{\frac{\Omega_{1}}{\sigma_{3}}} d n\left(\sqrt{\frac{\Omega_{3} \sigma_{2}}{\sigma_{1} \sigma_{3}}} \sigma_{2} t, k\right)
\end{aligned}
$$

onde as funções $c n(\tau, k)$ e $d n(\tau, k)$ são funções elípticas de Jacobi definidas por $c n(\tau, k)=$ $\sqrt{1-s n^{2}(\tau, k)}$ e $d n(\tau, k)=\sqrt{1-k^{2} s n^{2}(\tau, k)}$.

As funções elípticas de Jacobi são funções transcendentais duplamente periódicas, onde um dos períodos de oscilação das funções sn e $c n$ é $4 K$ e um dos períodos da função $d n$ é $2 K$, onde $K$ é dado pela seguinte expressão (Whittaker e Watson, 1950):

$$
K=\int_{0}^{1} \frac{d s}{\sqrt{\left(1-t^{2}\right)\left(1-k^{2} t^{2}\right)}}
$$

Além disso, as funções $s n$, $c n$ e $d n$, no limite $k \rightarrow 0$, resultam nas funções trigonométricas sin, cos e 1, respectivamente (Whittaker e Watson, 1950).

Portanto, pela análise da estabilidade linear mostrou-se que um tripleto conservativo deve apresentar um comportamento explosivo, entretanto, a solução analítica, quando $\sigma_{1}=\sigma_{2}=\sigma_{3}$, deve ser uma solução periódica. Nesse sentido, podemos afirmar que a não-linearidade impede que a solução tenha um crescimento exponencial e passe a ter um comportamento periódico.

Este resultado também é valido quando as amplitudes são funções complexas do tempo (Craik, 1988). Vale notar que a condição de ressonância utilizada por Craik (1988) é $\sigma_{1}+\sigma_{2}+\sigma_{3}=0$, e portanto, a condição para que a solução seja periódica torna-se $\sigma_{1}=1$ e $\sigma_{i}=-1, i=2,3$, ou $\sigma_{1}=-1$ e $\sigma_{i}=1, i=2,3$. 


\subsection{Resultados Numéricos}

Nesta seção será efetuada a integração numérica do sistema de equações diferenciais 4.16)-4.18 para o tripleto ressonante determinado no item anterior desta seção, composto por dois modos acústico-inerciais e um modo gravito-inercial. Para isso é preciso calcular primeiramente os coeficientes de interação $\alpha_{a}^{b c}, \alpha_{b}^{c a}$ e $\alpha_{a}^{b c}$ e as normas $E_{a}, E_{b}$ e $E_{c}$. Além disso, como o sistema de equações diferenciais é de primeira ordem, é preciso conhecer a amplitude no instante inicial de cada de modo $A_{a}^{0}, A_{b}^{0}$ e $A_{c}^{0}$. A integração numérica foi feita utilizando o método Leap-Frog com passo de tempo de um segundo. Uma vez que o sistema está resolvido, a identidade de Parseval (4.23) será utilizada para calcular a energia de cada modo em cada instante de tempo.

Utilizando o método descrito na seção 4.4 foi possível determinar um tripleto ressonante composto por dois modos acústico-inerciais e um modo gravito-inercial (Tabela 4.1). Uma vez determinado o tripleto é possível calcular os coeficientes de interação $\alpha_{b c}^{a}, \alpha_{c a}^{b}$ e $\alpha_{a b}^{c}$, que são dadas pela expressão 4.19) e os valores numéricos dos coeficientes estão na Tabela 4.2 .

Para poder integrar o sistema (4.16)-(4.18) é preciso conhecer os valores da energia de cada um dos modos. Essas energias são calculadas conforme descrito na Seção 3.1.6 do capítulo anterior. Na Tabela 4.3 temos o valor das energias dos modos que compõem o tripleto ressonante da Tabela 4.1. Ambos os cálculos dos coeficientes de interação e das normas dos modos do tripleto ressonante foram feitos através do cálculo numérico das integrais envolvidas nesses cálculos, onde utilizou-se o método de Simpson para a integral na vertical e da quadratura de Gauss para a integral no domínio meridional.

Tabela 4.1 - Valores do número de onda zonal, índice meridional, modo vertical e o tipo de onda de cada um dos elementos do tripleto analisado, assim como suas respectivas frequências temporais. Os modos são caracterizados, da esquerda para direita, pelo número de onda zonal, índice meridional, modo vertical e o tipo de onda, i.e., acústico-inercial (AI) ou gravito-inercial(GI). A frequência $\sigma$ dos modos está em cHz $\left(10^{-2} \mathrm{~Hz}\right)$. Os valores das frequências apresentam três algarismos significativos para ressaltar o fato de que o tripleto satisfaz aproximadamente a condição de ressonância.

\begin{tabular}{cccccc}
\hline modo d & modo b & modo c & $\sigma_{a}$ & $\sigma_{b}$ & $\sigma_{c}$ \\
\hline $476,0,1$, AI & $1,0,1, A I$ & $475,0,2, G I$ & 6.293 & 5.888 & 0.407 \\
\hline
\end{tabular}


Tabela 4.2 - Valor numérico dos coeficientes de interação dos modos que compõem o tripleto ressonante da Tabela 4.1 .

\begin{tabular}{ccc}
$\alpha_{d}^{b c}$ & $\alpha_{b}^{c d}$ & $\alpha_{c}^{d b}$ \\
\hline 402949545412.77 & 376739147812.07 & 26099317285.21 \\
\hline
\end{tabular}

Tabela 4.3 - Valor numérico das normas (em Joules) dos modos que compõem o tripleto ressonante da Tabela 4.1 .

\begin{tabular}{ccc}
\hline$E_{a}$ & $E_{b}$ & $E_{c}$ \\
\hline $1.3 \times 10^{10}$ & $1.3 \times 10^{20}$ & $7.02 \times 10^{6}$ \\
\hline
\end{tabular}

Por fim, é preciso definir os valores das amplitudes iniciais, i.e., $A_{a}(t=0), A_{b}(t=0)$ e $A_{c}(t=0)$. Os valores das amplitudes foram escolhidos de tal forma que os valores dos campos das perturbações de velocidade e pressão apresentassem valores fisicamente razoáveis. Para estimar os valores das amplitudes foram definidos os valores máximos para os campos de vento e pressão associados à essas amplitudes, com base na estrutura espacial dos modos constituintes do tripleto ressonante. Além disso, foram escolhidos os valores das amplitudes inicias de tal forma a explorar o comportamento das soluções em função do parâmetro $\tilde{m}$ definido em 4.20 .

As amplitudes iniciais do modos acústico-inercial curto (modo a), acústico-inercial longo (modo $b$ ) e gravito-inercial (modo $c$ ) utilizadas nos três experimentos são mostradas nas Tabelas 4.4, 4.6 e 4.8, assim como os máximos valores dos campos de vento e pressão na superfície da Terra $(z=0)$ associados com essas amplitudes inicias. As Tabelas 4.5, 4.7 e 4.9 referem-se aos complementos das Tabelas 4.4, 4.6 e 4.8, prespectivamente, mostrando os máximos dos campos do vento e pressão no topo da atmosfera. Nas Tabelas 4.4, 4.5, 4.6, 4.7, 4.8 e 4.9 é possível ver que os valores máximos das pertubações dos campos de velocidade e pressão são fisicamente razoáveis. No primeiro experimento, ilustrado na Figura 4.2 as amplitudes iniciais foram escolhidas para obter valores de $\tilde{m}$ no regime $0 \ll \tilde{m}<1$. No segundo experimento, ilustrado na Figura 4.3 , foi estudado o regime em que $0<\tilde{m} \ll 1$ e no terceiro experimento, ilustrado na Figura 4.4, as amplitudes foram escolhidas para obtermos um regime intermediário $0<\tilde{m}<1$. 
É interessante notar que nos três experimentos a energia do modo gravito-inercial variou muito menos que a energia dos outros dois modos acústico-inerciais. Ou seja, o modo gravito-inercial permite que os dois modos acústico-inerciais troquem energia, mas sua energia é pouco afetada pelas duas ondas acústico-inerciais. A principal diferença entre os três experimentos é o período das trocas de energia entre os modos. Outro aspecto interessante é que a energia total do tripleto é conservada no tempo.

No primeiro experimento $(0 \ll \tilde{m}<1)$ o modo acústico-inercial $a$ tem um aumento de energia abrupto e mantém-se constante por aproximadamente quatro dias. Depois desse período sua energia decai abruptamente voltando ao estado inicial. A energia do modo acústico-inercial $b$ possui um comportamento contrário ao comportamento do modo $a$. É interessante notar que durante o período em que a energia do modo a está constante ele também retém toda a energia do tripleto.

No segundo experimento $(0<\tilde{m} \ll 1)$ o período de troca de energia é de aproximadamente um dia, i.e. muito menor que o período do primeiro experimento. Além disso, o modo que retém a maior parte da energia é o modo gravito-inercial.

No terceiro experimento $(0<\tilde{m}<1)$ vemos um comportamento intermediário entre os dois outros experimentos. O modo gravito-inercial não retém a maior parte da energia, como no segundo experimento e o período de troca de energia é de aproximadamente uma semana, i.e. maior que o do segundo experimento, mas ainda é muito menor que o do primeiro experimento.

Para explorar as implicações físicas dessas trocas de energia entre os modos acústicoinerciais por meio do modo gravidade-inercial analisou-se a evolução temporal das pertubações do campo de vento zonal e vertical associadas ao modo $c$ (gravito-inercial) e ao modo a (modo acústico-inercial), respectivamente. Esta escolha foi feita baseada na energética dos modos normais do problema linearizado, pois os modos acústico-inerciais retém a maior parte de sua energia na forma de energia cinética vertical enquanto os modos gravito-inerciais retém a maior parte de sua energia na forma de energia cinética zonal. Além disso, foi levado em consideração que o modo a possui uma altura equivalente de 72156.82 metros enquanto, o modo $c$ possui altura equivalente de 303.15 metros. Portanto o modo $c$ é mais equatorialmente confinado que o modo $a$, pois, de acordo com a teoria da Maré de Laplace que descreve a estrutura meridional dos modos normais Kasahara, 1976, 1977), modos com altura equivalente menores são mais equatorialmente confinados, 
enquanto modos com altura equivalente maiores que a altura equivalente do modo externo $\left(H_{e}=10 \mathrm{~km}\right)$ possuem uma estrutura mais global. Quanto à estrutura vertical, o modo a possui a estrutura do primeiro modo baroclínico $(k=1)$ e, portanto, a máxima contribuição deste modo na vertical será no nível $z=0.5 z_{T}$. Por outro lado, o modo $c$ possui a estrutura vertical do segundo modo baroclínico $(k=2)$ e, portanto, a máxima contribuição deste modo na vertical será nos níveis $z=0.25 z_{T}$ e $z=0.75 z_{T}$. Utilizando as condições iniciais do primeiro experimento numérico e baseado nos aspectos mencionados acima decidimos analisar a evolução temporal do campo de vento zonal gerado pelo modo $c$ na região equatorial e no nível vertical $z=9000$ metros e analisamos a evolução temporal do campo de vento vertical gerado pelo modo a na latitude $\phi=10^{\circ} S$ e no nível vertical $z=4500$ metros. Na Figura 4.5 é possível ver que as amplitudes das oscilações de alta frequência das perturbações dos campos de vento zonal e vertical, associadas com a propagação de fase $\operatorname{dos} \operatorname{modos} b$ e $a$, são moduladas pelas trocas de energia (Figura 4.2 entre esses modos do tripleto ressonante. Vale ressaltar que as oscilações dentro do envoltório associadas com a propagação de fase dos modos não são visíveis na escala de tempo da Figura 4.5, porque os modos acústico-inerciais e gravito-inerciais aqui analisados são modos de altíssima frequência, com períodos de $15.8 \mathrm{~s}, 16.98 \mathrm{~s}$ e $245.75 \mathrm{~s}$.

Tabela 4.4 - Valores das amplitudes iniciais utilizadas no experimento numérico ilustrado na Figura 4.2 As variáveis $u_{s}, w_{s}$ e $p_{s}$ representam os maiores valores, em superfície, das pertubações da velocidade horizontal, vertical e pressão, respectivamente. Esses valores estão associados à amplitude inicial de cada um dos modos. As velocidades estão em m/s e a pressão está em hPa

\begin{tabular}{|l|l|l|l|l|}
\hline Modo & $A^{0}$ & $u_{s}$ & $w_{s}$ & $p_{s}$ \\
\hline $\mathrm{d}$ & 0.00 & 0.00 & 0.00 & 0.00 \\
$\mathrm{~b}$ & $1.79 \times 10^{-8}$ & $2.79 \times 10^{-3}$ & 2.55 & $1.07 \times 10^{1}$ \\
$\mathrm{c}$ & $2.04 \times 10^{-2}$ & $6.55 \times 10^{-1}$ & $1.36 \times 10^{-1}$ & $5.17 \times 10^{-1}$ \\
\hline
\end{tabular}


Tabela 4.5 - Esta tabela refere-se ao experimento ilustrado na Figura 4.2 e é apenas a continuação da Tabela 4.4. As variáveis $u_{T}, w_{T}$ e $p_{T}$ são os maiores valores, no topo da atmosfera, das perturbações da velocidade horizontal, vertical e pressão, respectivamente. As velocidades estão em m/s e a pressão está em hPa. E $\tilde{m}$ é o parâmetro dado pela expressão 4.20.

\begin{tabular}{|l|l|l|l|l|}
\hline Modo & $u_{T}$ & $w_{T}$ & $p_{T}$ & $\tilde{m}$ \\
\hline $\mathrm{d}$ & 0.00 & 0.00 & 0.00 & 0.99 \\
$\mathrm{~b}$ & $9.58 \times 10^{-3}$ & 9.00 & 3.04 & \\
$\mathrm{c}$ & 2.31 & $4.80 \times 10^{-1}$ & $1.46 \times 10^{-1}$ & \\
\hline
\end{tabular}

Tabela 4.6 - Valores das amplitudes iniciais utilizadas no experimento numérico ilustrado na Figura 4.3 As variáveis $u_{s}, w_{s}$ e $p_{s}$ representam os maiores valores, em superfície, das pertubações da velocidade horizontal, vertical e pressão, respectivamente. Esses valores estão associados à amplitude inicial de cada um dos modos. As velocidades estão em m/s e a pressão está em hPa

\begin{tabular}{|l|l|l|l|l|}
\hline Modo & $A^{0}$ & $u_{s}$ & $w_{s}$ & $p_{s}$ \\
\hline$a$ & 0.00 & 0.00 & 0.00 & 0.00 \\
$b$ & $1.08 \times 10^{-8}$ & $1.70 \times 10^{-3}$ & 1.55 & 6.53 \\
$c$ & $9.35 \times 10^{-2}$ & 3.00 & $6.22 \times 10^{-1}$ & 2.37 \\
\hline
\end{tabular}

Tabela 4.7 - Esta tabela refere-se ao experimento ilustrado na Figura 4.3 e é apenas a continuação da Tabela 4.6. As variáveis $u_{T}, w_{T}$ e $p_{T}$ são os maiores valores, no topo da atmosfera, das perturbações da velocidade horizontal, vertical e pressão, respectivamente. As velocidades estão em $\mathrm{m} / \mathrm{s}$ e a pressão está em hPa. E $\tilde{m}$ é o parâmetro dado pela expressão 4.20 .

\begin{tabular}{|l|l|l|l|l|}
\hline Modo & $u_{T}$ & $w_{T}$ & $p_{T}$ & $\tilde{m}$ \\
\hline$a$ & 0.00 & 0.00 & 0.00 & $4.76 \times 10^{-2}$ \\
$b$ & $6.00 \times 10^{-3}$ & 5.48 & 1.85 & \\
$c$ & $1.06 \times 10^{1}$ & 2.20 & $6.71 \times 10^{-1}$ & \\
\hline
\end{tabular}


Tabela 4.8 - Valores das amplitudes iniciais utilizadas no experimento numérico ilustrado na Figura 4.4 As variáveis $u_{s}, w_{s}$ e $p_{s}$ representam os maiores valores, em superfície, das pertubações da velocidade horizontal, vertical e pressão, respectivamente. Esses valores estão associados à amplitude inicial de cada um dos modos. As velocidades estão em $\mathrm{m} / \mathrm{s}$ e a pressão está em $\mathrm{hPa}$

\begin{tabular}{|l|l|l|l|l|}
\hline Modo & $A^{0}$ & $u_{s}$ & $w_{s}$ & $p_{s}$ \\
\hline$a$ & 0.00 & 0.00 & 0.00 & 0.00 \\
$b$ & $1.78 \times 10^{-8}$ & $2.79 \times 10^{-3}$ & 2.55 & $1.07 \times 10^{1}$ \\
$c$ & $2.65 \times 10^{-2}$ & $8.52 \times 10^{-1}$ & $1.77 \times 10^{-1}$ & $6.73 \times 10^{-1}$ \\
\hline
\end{tabular}

Tabela 4.9 - Esta tabela refere-se ao experimento ilustrado na Figura 4.4 e é apenas a continuação da Tabela 4.8. As variáveis $u_{T}, w_{T}$ e $p_{T}$ são os maiores valores, no topo da atmosfera, das perturbações da velocidade horizontal, vertical e pressão, respectivamente. As velocidades estão em m/s e a pressão está em hPa. E $\tilde{m}$ é o parâmetro dado pela expressão 4.20 .

\begin{tabular}{|l|l|l|l|l|}
\hline Modo & $u_{T}$ & $w_{T}$ & $p_{T}$ & $\tilde{m}$ \\
\hline$a$ & 0.00 & 0.00 & 0.00 & 0.59 \\
$b$ & $9.58 \times 10^{-3}$ & 9.00 & 3.04 & \\
$c$ & 3.01 & $6.24 \times 10^{-1}$ & $1.91 \times 10^{-1}$ & \\
\hline
\end{tabular}




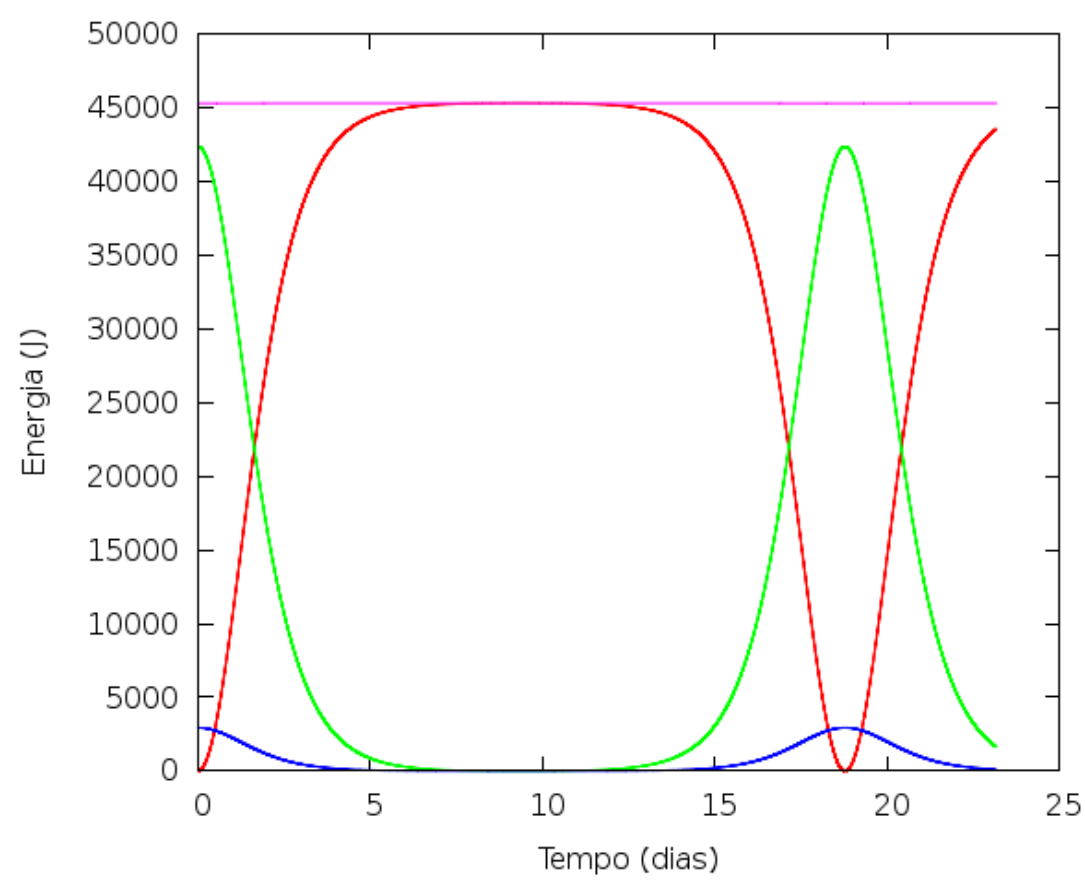
modo a $(476,0,1, \mathrm{Al})$
modo $b(1,0,1, A l)$
modo c $(475,0,2, \mathrm{Gl})$
Energia Total

Figura 4.2: Nesta figura temos as trocas de energias de um tripleto ressonante referentes ao primeiro experimento $(0 \ll \tilde{m}<1)$. Note que o modo $a$ da figura representa o modo $a$ do texto.

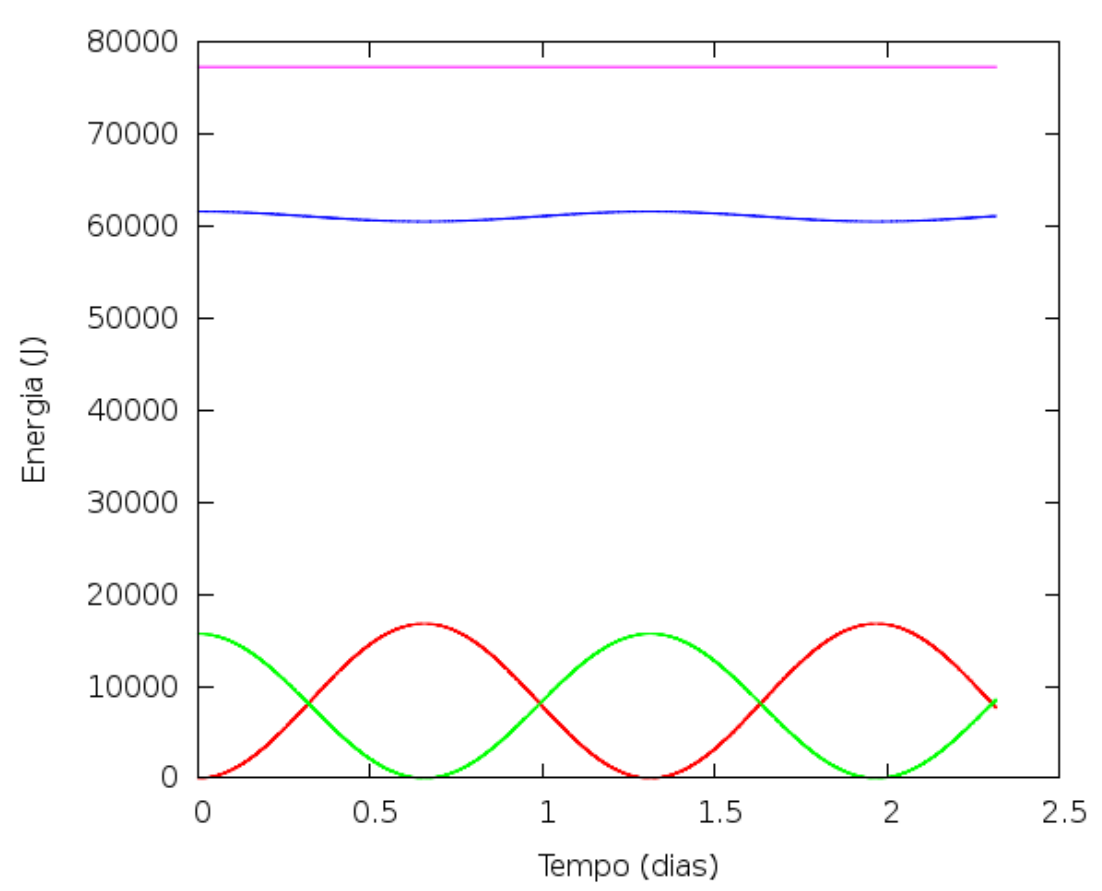
modo a $(476,0,1, \mathrm{Al})$
modo $b(1,0,1, \mathrm{Al})$
modo c $(475,0,2, \mathrm{Gl})$
Energia Total

Figura 4.3: Nesta figura temos as trocas de energias de um tripleto ressonante referentes ao segundo experimento $(0<\tilde{m} \ll 1)$. Note que o modo $a$ da figura representa o modo $a$ do texto. 


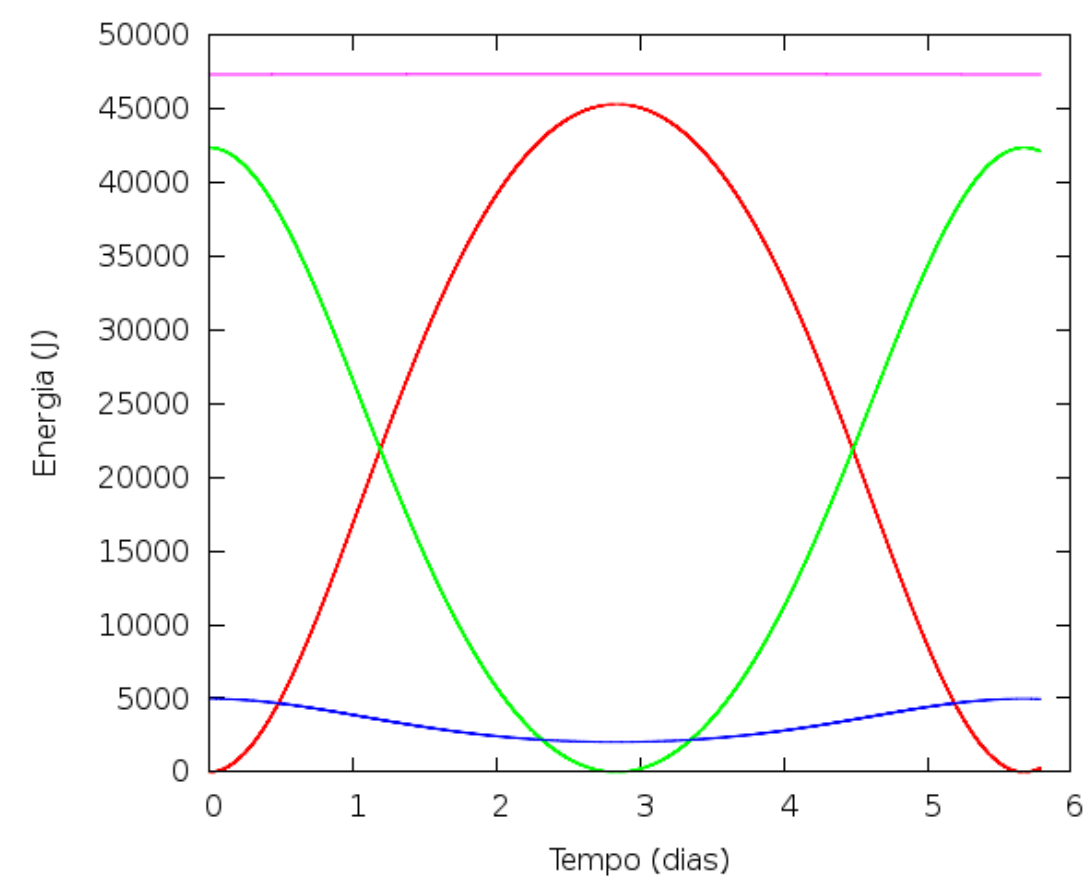

$$
\begin{array}{r}
\text { modo a }(476,0,1, \mathrm{Al}) \\
\text { modo } \mathrm{b}(1,0,1, \mathrm{Al}) \\
\text { modo c }(475,0,2, \mathrm{GI}) \\
\text { Energia Total }
\end{array}
$$

Figura 4.4: Nesta figura temos as trocas de energias de um tripleto ressonante referentes ao terceiro experimento $(0<\tilde{m}<1)$. Note que o modo $a$ da figura representa o modo $a$ do texto. 


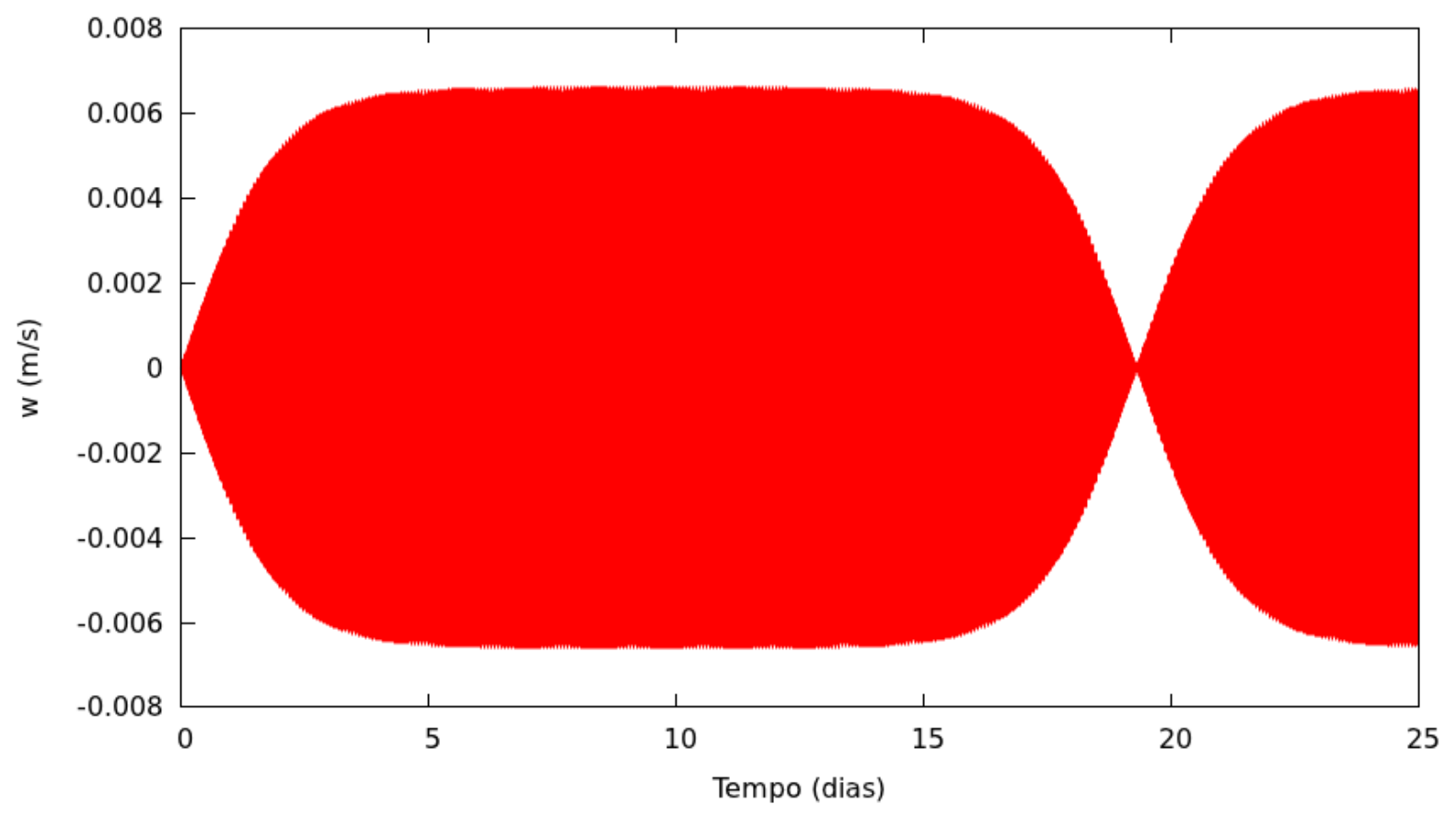

(a)

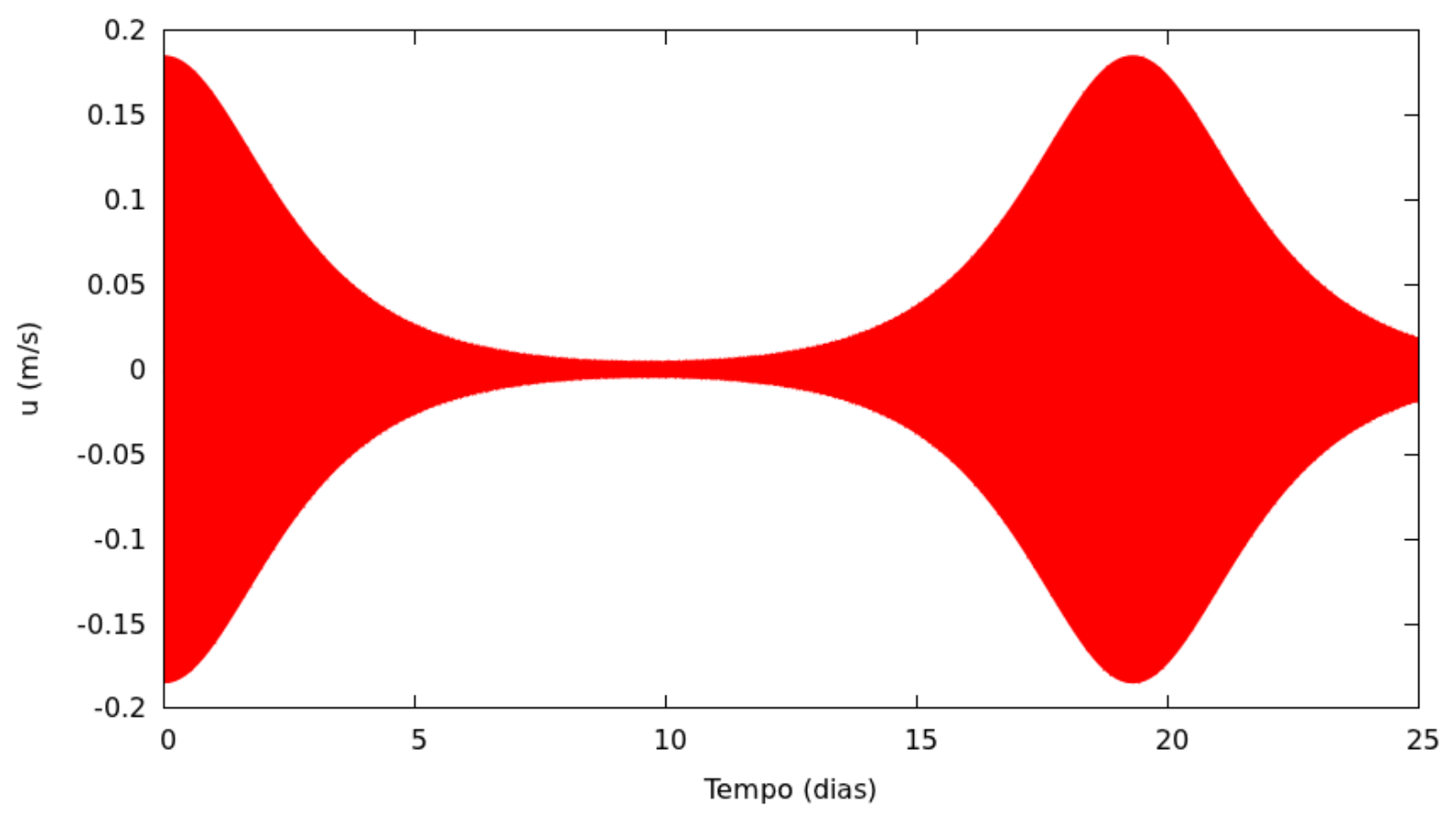

(b)

Figura 4.5: Evolução temporal da perturbação do campo do vento vertical em $\phi=10^{\circ} \mathrm{S}$, no nível vertical $z=9000 \mathrm{~m}$ e da perturbação do campo do vento zonal em $\phi=0^{\circ}$, no nível vertical $z=4500 \mathrm{~m}$. A velocidade está em $\mathrm{m} / \mathrm{s}$ e o tempo está em dias. Não é possível ver a oscilação interna ao envoltório devido a escala de tempo do gráfico. 
Capítulo 5

\section{Conclusões}

Neste trabalho, com o modelo e a metodologia proposta por Kasahara e Qian (2000), foi realizado um estudo sobre a energética de um modelo global, não-hidrostático, compressível e baroclínico.

Uma das principais diferenças entre modelo das equações primitivas (Phillips, 1990) e a versão não hidrostática desse modelo (Kasahara e Qian, 2000), é a presença dos modos acústico-inerciais no modelo não-hidrostático. A energia dos modos acústico-inerciais está concentrada nas energias cinética vertical e na energia elástica. Além disso, a energia cinética vertical aumenta, enquanto a energia elástica diminui com o aumento do número de onda zonal $s$. A energética dos modos gravito-inerciais no caso não-hidrostático apresentou muita semelhança com a energética dos modos gravito-inerciais no caso hidrostático. A diferença entre os dois casos começam a aparecer para modos com número de onda zonal maiores, ou seja, para modos mais curtos. Este último resultado era esperado, pois a relação de dispersão dos modos gravito-inerciais não-hidrostáticos diferencia-se do caso hidrostático, apenas para os modos mais curtos, onde o módulo da frequência dessas ondas é limitada superiormente pela frequência de Brunt-Väisälä (Kasahara e Qian, 2000).

Além da energética linear, foi realizado um estudo das interações fracamente nãolineares permitidas pelo modelo. Mais especificamente, analisou-se as trocas de energia de um tripleto ressonante composto por dois modos acústico-inerciais e um modo gravitoinercial. Primeiramente as variáveis dinâmicas foram representadas por um estado básico hidrostático e isotérmico sobreposto por uma perturbação. Mas diferentemente da análise feita por Kasahara e Qian (2000), os termos de segunda ordem foram retidos no sistema. Para resolver o sistema contendo as não-linearidades quadráticas utilizou-se o método de Galerkin, com as soluções características do sistema linearizado atuando como funções 
base. Analisando a dinâmica reduzida para um único tripleto ressonante composto por dois modos acústico-inerciais e um modo gravito-inercial, foi possível mostrar que modos de altíssima frequência (com períodos de aproximadamente 15 segundos) podem realizar trocas de energia numa escala de tempo muito maior que o período associado com a propagação de fase desses modos. A escala de tempo das trocas de energia do tripleto estudado variou desde valores próximos ao ciclo diurno (Figura 4.3) até valores de quase vinte dias (Figura 4.2). Além disso, mostrou-se que essas trocas de energia modulam as oscilações de alta frequência nas perturbações dos campos de vento e pressão associadas com a propagação de fase dos modos acústico-inerciais. Portanto, neste modelo simplificado, foi possível mostrar que os modos acústico-inerciais, que são modos de altíssima frequência e que costumam ser filtrados e/ou eliminados dos modelos numéricos de previsão do tempo, podem influenciar fenômenos de escala sinótica e até fenômenos de escala intrasazonal, através da interação triádica entre dois modos acústico-inerciais e um modo gravito-inercial.

Em trabalhos futuros é possível estudar a energética dos modos normais modelo, considerando os modos com topo atmosférico $z_{T} \rightarrow \infty$, que corresponde às autofunções com modo vertical $k=0$ e estudar as interações fracamente não-lineares para este caso. Como no caso do topo atmosférico infinito, as curvas de dispersão dos modos acústico-inerciais e gravito-inerciais aproximam-se mais da frequência de Brunt-Väisälä, existe a possibilidade de que um modo de Rossby interaja com um modo acústico-inercial e um modo gravito-inercial, formando um tripleto quase-ressonante, ou seja, um tripleto que satisfaz as condições de ressonância de forma aproximada. Outra possibilidade de estudo é a utilização do formalismo Hamiltoniano para descrever o sistema de equações considerado neste trabalho. Ao utilizar o formalismo Hamiltoniano a relação entre simetrias e leis de conservação torna-se explícita, o que facilita o estudo de quantidades conservadas pelo sistema físico em estudo (Goldstein, 1980, Landau e Lifshitz, 1976). A aplicação do formalismo Hamiltoniano em fluidos requer um refinamento da matemática envolvida Arnol'd, 1989: Shepherd, 1990; Olver, 2000). O formalismo Hamiltoniano foi utilizado com sucesso em problemas de dinâmica de fluidos geofísicos (Salmon, 1998; Morrison, 1982; Scinocca e Shepherd, 1992; Ripa et al., 2003). Além disso, com o formalismo Hamiltoniano é possível aprofundar o estudo das interações não-lineares entre os modos normais do modelo em estudo (Bustamante e Kartashova, 2009; Kartashova, 2011). 


\section{Referências Bibliográficas}

Abarbanel H., Rabinovich I., Introduction to Nonlinear Dynamics for Physicists. Lecture Notes in Physics Series, World Scientific, 1993

Arnol'd V., Mathematical Methods of Classical Mechanics. Graduate Texts in Mathematics, Springer, 1989

Bonatti J. P., Silva Dias P. L., 1983 Relatório Externo INPE-2674-RPE/428 Um Modelo Espectral Barotrópico Global com Iniciação por Modos Normais. Instituto Nacional de Pesquisas Espaciais

Bonatti J. P., Silva Dias P. L., Moura A. D., 1983 Relatório Externo INPE-2578-PRE/226 Funções de Hough: Teoria e Utilização. Instituto Nacional de Pesquisas Espaciais

Bretherton F. P., Low frequency oscillations trapped near the equator, Tellus A, 1964, vol. 16 , p. 181

Brown A., Milton S., Cullen M., Golding B., Mitchell J., Shelly A., Unified Modeling and Prediction of Weather and Climate: A 25-Year Journey, Bull. Amer. Meteor. Soc., 2012, vol. 93 , p. 1865

Bustamante M. D., Kartashova E., Dynamics of nonlinear resonances in Hamiltonian systems, Europhysics Letters, 2009, vol. 85

Chapman S., Lindzen R. S., Atmospheric Tides: Thermal and Gravitational. Gordon and Breach, 1970

Craik A., Wave Interactions and Fluid Flows. Cambridge Monographs on Mechanics, Cambridge University Press, 1988 
Daley R., The normal modes of the spherical non-hydrostatic equations with applications to the filtering of acoustic modes, Tellus, 1988, vol. 40

Davies T., Staniforth A., Wood N., Thuburn J., Validity of anelastic and other equation sets as inferred from normal-mode analysis, Q. J. R. Meteorol. Soc., 2003, vol. 129, p. 2761

Draghici I., The hypothesis of a marginally shallow atmosphere, Sov. Meteorol. Hydrol., 1989, vol. 19 , p. 13

Drazin P., Reid W., Hydrodynamic Stability. Cambridge University Press, 1981

Dudhia J., Bresch J. F., A Global Version of the PSU-NCAR, Mon. Wea. Rev., 2002, vol. 130 , p. 2989

Eckart C., Hydrodynamics of Oceans and Atmosphere. Pergamon Press, 1960

Fruman M. D., Shepherd T. G., Symmetric Stability of Compressible Zonal Flows on a Generalized Equatorial $\beta$ Planes, J. Atmos. Sci., 2008, vol. 65, p. 1927

Gill A., Atmosphere-Ocean Dynamics. Academic Press, 1982

Giraldo F. X., Restelli M., Läuter M., Semi-Implicit Formulations of the Navier-Stokes Equations: Application to Nonhydrostatic Atmospheric Modeling, SIAM J. Sci. Comput., 2010, vol. 32 , p. 3394

Goldstein H., Classical mechanics. Addison-Wesley series in physics, Addison-Wesley Pub. Co., 1980

Hayashi M., Itano T., The Importance of the Nontraditional Coriolis Terms in Large-Scale Motions in the Tropics Forced by Prescribed Cumulus Heating, J. Atmos. Sci., 2012, vol. 69 , p. 2699

Hough S. S., On the applications of harmonic analysis to the dynamical theory of the tide-Part II. On the general integration of Laplace's dynamical equations, Philo. Trans. Roy. Soc. London, 1898, pp 139-185

Itano T., Kasahara A., Effect of Top and Bottom Boundary Conditions on Symmetric Instability under Full-Component Coriolis Force, J. Atmos. Sci., 2011, vol. 68, p. 2771 
Kartashova E., Nonlinear Resonance Analysis. Cambridge University Press, 2011

Kasahara A., Normal modes of ultralong waves in the atmosphere, Mon. Wea. Rev., 1976, vol. 104, p. 669

Kasahara A., Numerical integration of the global barotropic primitive equations with Hough harmonics expansions, J. Atmos. Sci., 1977, vol. 34, p. 687

Kasahara A., Further studies on a spectral model of the global barotropic primitice equations with Hough harmonics expansions, J. Atmos. Sci., 1978, vol. 35, p. 2043

Kasahara A., On the nonhydrostatic atmospheric models with inclusion of the horizontal component of the Earth's angular velocity, J. Meteorol. Soc. Japan, 2003a, vol. 81, p. 935

Kasahara A., The roles of the horizontal componente of the Earth's angular velocity in nonhydrostatic linear models, J. Atmos. Sci., 2003b, vol. 60, p. 1085

Kasahara A., , 2004 Technical Report NCAR/TN-457+STR Free Oscillations of Deep Nonhydrostatic Global Atmosphere: Theory and a Test of Numercial Schemes. National Center for Atmospheric Research

Kasahara A., Gary J. M., Normal modes of an incompressible and stratified fluid model including the vertical and horizontal componentes of Coriolis force, Tellus A, 2006, vol. 58, p. 368

Kasahara A., Gary J. M., Studies of inertio-gravity waves on midlatitude beta-plane without the traditional approximation, Q. J. R. Meteorol. Soc., 2010, vol. 136, p. 517

Kasahara A., Qian J.-H., Normal Modes of a Global Nonhydrostatic Atmospheric Model, Mon. Wea. Rev., 2000, vol. 128, p. 3357

Khouider B., Majda A. J., Stechmann S. N., Climate Science in the Tropics: Waves, Vortices, asnd PDEs, Nonlinearity, 2013, vol. 26, p. 1

Klein R., Asymptotics, Structure, and Integration of Sound-Proof Atmospheric Flow Equations, Theor. Comput. Fluid Dyn, 2009, vol. 23, p. 161 
Klemp J. B., Skamarock W. C., Dudhia J., Conservative Split-Explicit Time Integration Methods for the Compressible Nonhydrostatic Equations, Mon. Wea. Rev., 2007, vol. 135 , p. 2897

Landau L., Lifshitz E., Mechanics. Butterworth Heinemann, Butterworth-Heinemann, 1976

Longuet-Higgins M. S., The eigenfuntions of Laplace's tidal equation over a sphere, Proc. Roy. Soc. London, 1968, pp 511-607

Lynch P., The origins of computer weather prediction and climate modeling, J. Comput. Phys., 2008, vol. 227, p. 3431

Majda A., Introduction to Pdes and Waves for the Atmosphere and Ocean. Courant Lecture Notes in Mathematics Series, Courant Institute of Mathematical Sciences, New York University, 2003

Marshall J., Perelman L., Adcroft A., Hydrostatic, quasi-hydrostatic, and nonhydrostatic ocean modeling, J. Geophys. Res., 1997, vol. 102, p. 5733

Matsuno T., Quasi-geostrophic motions in the equatorial area, J. Meteor. Soc. Japan, 1966, vol. 44 , p. 25

Müller R., A note on the relation between the "traditional approximation" and the metric of the primitive equations, Tellus A, 1989, vol. 41, p. 175

Morrison P. J., Poisson Brackets for Fluids and Plasmas, Mathematical Methods in Hydrodynamics and Integrability in Related Dynamical SYstems, 1982, vol. 88, p. 13

Murakami H., Matsumura T., Development of an effective non-linear normal mode initialization method for a high resolution global model., J. Meteorol. Soc. Japan, 2007, vol. 85 , p. 187

Olver P., Applications of Lie groups to differential equations. Graduate Texts in Mathematics Series, Springer-Verlag, 2000

Pedlosky J., Geophysical fluid dynamics. Springer-Verlag, 1987

Phillips N. A., The oscillations of the atmosphere, World Meteor. Org., 1990 
Pielke R., Mesoscale Meteorological Modeling. International geophysics series, Academic Press, 2002

Press W. H., Teukolsky S. A., Vetterling W. T., Flannery B. P., Numerical Recipes in Fortran 77: The Art of Scientific Computing. Cambridge University Press, 1992

Raupp C. F. M., Interação não-linear entre ondas atmosféricas: um possível mecanismo para a conexão trópicos-extratrópicos em baixa-freqüência, IAG-USP, 2006, Tese de Doutorado, 133 pp.

Raupp C. F. M., Silva Dias P. L., Tabak E. G., Milewski P., Resonant Wave Interactions in the Equatorial Waveguide, J. Atmos. Sci., 2008, vol. 65, p. 3398

Ripa P., Fuentes O., Sheinbaum J., Ochoa J., Nonlinear Processes in Geophysical Fluid Dynamics: A Tribute to the Scientific Work of Pedro Ripa. Kluwer Academic Publishers, 2003

Salmon R., Lectures on Geophysical Fluid Dynamics. Oxford University Press, USA, 1998

Scinocca J. F., Shepherd T. G., Nonlinear Wave-Activity Conservarion Laws and Hamiltonian Structure for the Two-Dimensional Anelastic Equations, J. Atmos. Sci., 1992, vol. 49 , p. 339

Shepherd T. G., Symmetries, conservation lays, and Hamiltonian structure in geophysical fluid dynamics, Adv. Geophys, 1990, vol. 32, p. 287

Staniforth A., Wood N., Aspects of the dynamical core of a nonhydrostatic, deepatmosphere, unified weather and climate-prediction model, J. Comput. Phys., 2006, vol. 227 , p. 3445

Swarztrauber P. N., Kasahara A., The Vector Harmonic Analysis of Laplace's Tidal Equations, Europhysics Letters, 1985, vol. 6

Taylor G. I., The oscillations of the atmosphere, Proc. Roy. Soc. London, 1936, pp 318-224

Thuburn J., Wood N., Staniforth A., Normal modes of deep atmosphere. I: Spherical geometry, Q. J. R. Meteorol. Soc., 2002a, vol. 128, p. 1771 
Thuburn J., Wood N., Staniforth A., Normal modes of deep atmosphere. II: $f$-plane geometry, Q. J. R. Meteorol. Soc., 2002b, vol. 128, p. 1793

Vallis G., Atmospheric and Oceanic Fluid Dynamics: Fundamentals and Large-scale Circulation. Cambridge University Press, 2006

White A. A., Hoskins B. J., Roulstone I., Staniforth A., Consistent approximate models of the global atmosphere: shallow, deep, hydrostatic, quasi-hydrostatic and nonhydrostatic, Q. J. R. Meteorol. Soc., 2005, vol. 131, p. 2081

Whitham G., Linear and Nonlinear Waves. Pure and Applied Mathematics: A Wiley Series of Texts, Monographs and Tracts, Wiley, 1974

Whittaker E., Watson G., A Course of Modern Analysis. Cambridge University Press, 1950 